\title{
NEW $H$ (div)-CONFORMING MULTISCALE HYBRID-MIXED METHODS FOR THE ELASTICITY PROBLEM ON POLYGONAL MESHES
}

\author{
Philippe R. B. Devloo ${ }^{1}$, Agnaldo M. Farias ${ }^{2}$, Sônia M. Gomes ${ }^{3, *}$, \\ Weslley Pereira ${ }^{4}$, Antonio J. B. dos Santos $^{5}$ and Frédéric Valentin ${ }^{4}$
}

\begin{abstract}
This work proposes a family of multiscale hybrid-mixed methods for the two-dimensional linear elasticity problem on general polygonal meshes. The new methods approximate displacement, stress, and rotation using two-scale discretizations. The first scale level setting consists of approximating the traction variable (Lagrange multiplier) in discontinuous polynomial spaces, and of computing elementwise rigid body modes. In the second level, the methods are made effective by solving completely independent local boundary Neumann elasticity problems written in a mixed form with weak symmetry enforced via the rotation multiplier. Since the finite-dimensional space for the traction variable constraints the local stress approximations, the discrete stress field lies in the $H$ (div) space globally and stays in local equilibrium with external forces. We propose different choices to approximate local problems based on pairs of finite element spaces defined on affine second-level meshes. Those choices generate the family of multiscale finite element methods for which stability and convergence are proved in a unified framework. Notably, we prove that the methods are optimal and high-order convergent in the natural norms. Also, it emerges that the approximate displacement and stress divergence are super-convergent in the $L^{2}$-norm. Numerical verifications assess theoretical results and highlight the high precision of the new methods on coarse meshes for multilayered heterogeneous material problems.
\end{abstract}

Mathematics Subject Classification. 65N12, 65N15, 65N30, 74G15.

Received August 27, 2020. Accepted March 11, 2021.

\footnotetext{
Keywords and phrases. Multiscale, mixed finite elements, linear elasticity, hybridization.

1 Faculdade de Engenharia Civil, Arquitetura e Urbanismo, Universidade Estadual de Campinas, Rua Josiah Willard Gibbs, 85, Campinas, São Paulo 13083-839, Brazil.

2 Instituto Federal do Norte de Minas Gerais, Fazenda Varginha, km 02 Rodovia MG-404, Salinas, Minas Gerais 39560000, Brazil.

3 Instituto de Matemática, Estatística e Computação Científica, Universidade Estadual de Campinas, Rua Sérgio Buarque de Holanda, 651, Campinas, São Paulo 13083-859, Brazil.

4 Laboratório Nacional de Computação Científica, Petrópolis, Rio de Janeiro 25651-075, Brazil.

${ }^{5}$ Centro de Informática, Universidade Federal da Paraíba, R. dos Escoteiros, s/n - Mangabeira, João Pessoa - PB, João Pessoa, Paraíba 58055-000, Brazil.

*Corresponding author: soniag@unicamp.br
} 


\section{INTRODUCTION}

Mixed finite element (FE) methods for elasticity problems, based on the Hellinger-Reissner principle, have been used since the beginning of finite element history. They are formulated simultaneously for stress and displacement variables, which are of primary interest. Moreover, the importance of using hybridization in stress mixed formulations for elasticity problems has also been early recognized by the pioneer engineers in structural mechanics (e.g., see [38] and the citations therein, including the work by T. H. H. Pian). When correctly designed, stress mixed methods usually give optimal stress accuracy, and local momentum conservation. Moreover, they do not present locking behavior for incompressible or nearly incompressible materials.

We focus our study on conforming stress mixed formulations, meaning that approximations for the stress tensor $\sigma$ must have continuous normal traces (traction) along inter-element boundaries (i.e., the stress FE space should be $H$ (div)-conforming). The displacement variable $\underline{u}$ lives in a discontinuous space. These kinds of methods are formulated as minimization problems constrained by the realization of the divergence equation, and displacement plays the role of the corresponding Lagrange multiplier. However, as mentioned in [4], the divergence-consistency, a property required for the method to be well-posed, has proved to be surprisingly hard to be fulfilled by symmetric tensors and displacement FE pairs. There is another approach that does not assume symmetry in the tensor space from the beginning. Instead, the idea is to impose the symmetry condition in a weak form, which requires a stable choice of another FE space for the (multiplier) rotation variable $q$. We denote this class of methods by the acronym MFEM-WS, and refer to [3,22] for overviews on this matter.

Realistic problems in solid mechanics are frequently associated with domains with complex geometries, in the occurrence of fractures or heterogeneities in the materials, or under intricate types of loads. On the other hand, standard finite element methods need refined meshes to capture small structures in the data, which reflects an elevated computational cost. With this motivation, our purpose is to create a flexible multiscale hybrid approach for the MFEM-WS formulation. Our method is based on a divide-and-conquer strategy combined with bubble enrichment techniques and static condensation, which are general-purpose tools widely adopted in multiscale simulations. It shall be denoted by the acronym MHM-WS, for its design is in the spirit of Multiscale Hybrid Mixed (MHM) methods (already applied for Darcy problems [20,26], for displacement-based elasticity formulations $[28,35,36]$, and other contexts therein cited).

In summary, this means that the MHM-WS scheme shares with these MHM methods the following characteristics:

(1) It can be interpreted as a discrete version of a hybrid formulation characterizing the exact solution in terms of components given by well-posed local-global systems.

(2) There is a macro-partition $\mathcal{T}=\left\{\Omega_{i}\right\}$ of $\Omega$, and a set of local problems over each (general polygonal) subregion $\Omega_{i}$.

(3) A new normal trace variable (multiplier) is introduced over the subregion boundaries (mesh skeleton), making the referred inter-element connection.

(4) There are two-scale operators (upscaling and downscaling) transferring information between the two levels of resolution.

(5) There is an orthogonal decomposition of the potential (displacement) variable in terms of a coarse (piecewise rigid body motions), defined over $\mathcal{T}$, and a fine-scale components.

(6) As in usual static-condensation procedures, the multiplier and the coarse potential component are computed by a stable global system (upscaling stage).

(7) In the second fine scale, the small details of all variables are computed by a set of problems restricted to the subregions $\Omega_{i} \in \mathcal{T}$, taking the multiplier as Neumann boundary data over $\partial \Omega_{i}$, and using the adopted stable formulations for each one (downscaling stage). The local FE spaces may have richer internal resolution than the boundary traces. Each local problem is completely independent of the others since test functions have support inside a single subregion.

(8) The local downscaling problems favor the use of parallel strategies. 
We shall mention that this family of multiscale methods does not assume periodicity on the elastic coefficients nor separation of scales in its construction. Thereby, it can be used (formally) for general problems with heterogeneous coefficients.

For the current MHM-WS scheme, the approximate stress $\underline{\underline{\sigma}}$ is obtained in a FE space of tensors with normal traces strongly constrained to a given FE space over the mesh skeleton, where we search for the new multiplier variable $\underline{\tilde{\lambda}}$. Consequently, $\underline{\underline{\sigma}}$ is $H(\mathrm{div})$-conforming. Moreover, and to increase accuracy, we can enrich the tensor bubble functions (with support on a single subregion) using different strategies: refining the internal mesh, increasing the polynomial degree, or both. For neighboring subregions $\Omega_{i}$ and $\Omega_{j}$, their internal partitions $\mathcal{T}^{\Omega_{i}}$ and $\mathcal{T}^{\Omega_{j}}$ are allowed to be non-conformal over $\Gamma_{i, j}=\partial \Omega_{i} \cap \partial \Omega_{j}$. In principle, element geometry, mesh widths, and polynomial degrees in the subregions may vary. However, some mesh and space consistencies should be satisfied (see Sect. 3.1).

The strategy we present requires some stability conditions, namely divergence and Stokes constraints, of the two-scale tensor FE space with respect to displacement and rotation FE spaces. Under these circumstances, an important analysis aspect of the MHM-WS method is that it may be interpreted as an equivalent stable MFEMWS formulation of the model problem, both based on the same FE space framework. The divergence constraint is obtained by forming rows of tensors and displacements with Poisson-compatible FE pairs widely used for flux and potential approximations in mixed methods. Concerning the enforcement of the Stokes constraint, we extend the methodology proposed in [19] to construct new stable Stokes-compatible pairs: the pair used for stability analysis at the coarsest single-level space setting is incremented with extra refined composite bubble terms for the velocity in order to restore stability when using enlarged pressure spaces, in the spirit of the methodology suggested in [9]. Classical tools are applied to the equivalent two-scale MFEM-WS framework, guiding the error analysis of the MHM-WS solutions. We prove optimal and high-order convergence for displacement, stress and rotation unknowns in their natural norms under some regularity assumptions. Stress and rotation variables are approximated with the same accuracy order as for the trace variable. Notably, super-convergence in the $L^{2}$-norm for the divergence of the stress and enhanced displacement may be reached.

Recently, the authors in [30] pointed out that the resolution of elasticity problems by multiscale mixed stressdisplacement formulations, based on domain decomposition, had not been considered before. They proposed and analyzed a multiscale mixed formulation using the mortar domain decomposition with non-matching grids, and weakly imposed stress symmetry. The mortar spaces use displacement Lagrange multipliers to (weakly) enforce interface continuity of the normal stress. Following a similar divide-and-conquer principle but designed in the different MHM context, the MHM-WS method also fills this gap.

There are some other works that use multiscale FE methods to solve elasticity problems. In [11,12], the authors applied the Multiscale Finite Element Method (MsFEM) to solve an elasticity problem in a composite material. Each level in the MsFEM has its mesh and interpolation spaces that, in general, fit inside the interpolations of lower levels. The MsFEMs have no local problems associated with the source function neither a rigorous mathematical structure to guide the choice of local boundaries. The Heterogeneous Multiscale FE method (HMM) [1] discretizes the elasticity problem by a macroscopic FE method coupled with a microscopic FE method resolving the micro scales and recovering the macroscopic properties of the material. The Localized Orthogonal Decomposition (LOD) method of [32] is a multiscale method that requires low regularity on the variational problem. It avoids the use of additional regularity by computing the multiscale basis functions on a set (patch) of macro elements. A generalized FE method (GFEMs) using LOD is presented in [29].

\subsection{Outline of the paper}

Section 2 starts with the weak stress mixed formulation with reduced stress symmetry for the model problem, and a new hybrid local-global characterization of the exact solution. Discrete two-scale versions of these methods are presented in Section 3. For that, we construct a hierarchy of partitions and two-scale FE space settings $\mathcal{E}_{\gamma}$, the corresponding formulations being denoted by the acronym $\operatorname{MFEM}-\mathrm{WS}\left(\mathcal{E}_{\gamma}\right)$. The MHM-WS( $\left.\mathcal{E}_{\gamma}\right)$ schemes correspond to hybrid local-global versions of the MFEM-WS $\left(\mathcal{E}_{\gamma}\right)$. In Section 4, we establish the stability of the MHM-WS $\left(\mathcal{E}_{\gamma}\right)$ methods for two specific families of two-scale FE spaces $\mathcal{E}_{\gamma}$, for triangular and affine quadrilateral 
partitions. The error analysis of the $\operatorname{MHM}-\operatorname{WS}\left(\mathcal{E}_{\gamma}\right)$ solutions is performed in Section 5 . For that, we extend a typical analysis used for single-level methods to the more general two-scale FE settings $\mathcal{E}_{\gamma}$. We present the results of computational simulations in Section 6, confirming the predicted theoretical convergence results of Section 5. In the same section, we compare the results with the ones from the MHM- $H^{1}$ method, and with the results obtained with the classical coarse single-level MHM-WS methods. The final part of this section dedicates to numerical tests in heterogeneous media with high-contrast layers. After some concluding remarks in Section 7, Appendix A presents the proofs of some theorems previously stated.

\subsection{Some comments about notation}

We use various symbols for the notation of variables, data, geometric elements, finite element spaces, and nomenclature. For those wishing to keep them straight, they are listed in Appendix B. For instance, throughout this paper, for a region $D \subseteq \Omega, \underline{n}^{D}$ denotes the external unitary normal to $\partial D$. The scalar Hilbert spaces $L^{2}(D)$ and $H^{s}(D)$ have the usual meaning and norms. We also consider the spaces $L^{2}\left(D, \mathbb{R}^{2}\right), L^{2}(D, \mathbb{M}), H^{s}\left(D, \mathbb{R}^{2}\right)$, and $H^{s}(D, \mathbb{M})$, which inherit the corresponding norms associated to $L^{2}(D)$ and $H^{s}(D) . H(\operatorname{div}, D)$ is the usual space composed by square-integrable vector functions, for which the divergence is also square integrable. Similarly, we consider the space of tensor functions $H(\operatorname{div}, D, \mathbb{M})$, the divergence of a tensor field being the vector field obtained by taking the divergence of each row. Moreover, for a vector function $\underline{q}=\left[\begin{array}{l}q_{1} \\ q_{2}\end{array}\right], \underline{\underline{\nabla}} \times \underline{q}$ denotes the tensor $\underline{\underline{\nabla}} \times \underline{q}=\left[\begin{array}{l}\underline{\nabla} \times q_{1} \\ \underline{\nabla} \times q_{2}\end{array}\right]=\left[\begin{array}{l}\partial_{2} q_{1}-\partial_{1} q_{1} \\ \partial_{2} q_{2}-\partial_{1} q_{2}\end{array}\right]$.

We use the notation $(\cdot, \cdot)_{D}$ for the $L^{2}$-inner products, and $\langle\cdot, \cdot\rangle_{\partial D}$ refers to the duality pairing between $H^{1 / 2}\left(\partial D, \mathbb{R}^{2}\right)=\left\{\underline{\mu}=\left.\underline{u}\right|_{\partial D}, \underline{u} \in H^{1}\left(D, \mathbb{R}^{2}\right)\right\}$ and $H^{-1 / 2}\left(\partial D, \mathbb{R}^{2}\right)=\left\{\underline{\mu}=\left.\underline{\underline{\tau}} \underline{\underline{n}}^{D}\right|_{\partial D}, \underline{\underline{\tau}} \in H(\operatorname{div}, D, \mathbb{M})\right\}$. We drop the subscript $\bar{D}$ whenever $D=\Omega$.

\section{Stress mixed Formulation With REDUCED STRESS SyMmetry}

Let $\Omega \subset \mathbb{R}^{2}$ be a polygonal domain occupied by a linear elastic body. Given the body force $\underline{f}$ and Dirichlet boundary data $\underline{g}$, the equations of the static elasticity in the Hellinger-Reissner form determine that stress $\underline{\underline{\sigma}}$ and displacement $\underline{u}$ fields satisfy the following equilibrium and constitutive equations

$$
-\underline{\nabla} \cdot \underline{\underline{\sigma}}=\underline{f}, \quad \underline{\underline{\sigma}}=\underline{\underline{A}} \underline{\underline{\varepsilon}}(\underline{u}) \text { in } \Omega, \quad \underline{u}=\underline{g} \text { on } \partial \Omega,
$$

where $\underline{\underline{\varepsilon}}(\underline{u})=\frac{\nabla \underline{u}+\nabla \underline{u}^{T}}{2}$ is the infinitesimal strain tensor. The given data are $\underline{g} \in H^{\frac{1}{2}}\left(\partial \Omega, \mathbb{R}^{2}\right)$ and $\underline{f} \in$ $L^{2}\left(\Omega, \mathbb{R}^{2}\right)$. The material properties are described by the stiffness tensor $\underline{\underline{A}}=\underline{\underline{A}}(x, y)$ for all $(x, y) \in \mathbb{R}^{2}$, which is a self-adjoint, bounded, and uniformly positive definite linear operator acting on the set of symmetric tensors $\mathbb{S}$. We assume that $\underline{\underline{A}}$ can be extended to general second-order tensors $\mathbb{M}=\mathbb{R}^{2 \times 2}$ with the same properties. In particular, in the case of an isotropic body, $\underline{\underline{A}} \underline{\underline{\varepsilon}}=2 \mu \underline{\underline{\varepsilon}}+\lambda \operatorname{tr}(\underline{\underline{\varepsilon}}) \underline{\underline{I}}$, where $\lambda$ and $\mu$ are the Lamé parameters, and $\underline{\underline{I}}$ is the $2 \times 2$ identity matrix. Both the material properties $\underline{\underline{A}}$ and the given source data $\underline{f}$ may be heterogeneous and embed various length scales.

\subsection{The weak formulation}

Problem (2.1) admits an equivalent expression, without assuming stress symmetry a priori, by replacing the original constitutive equation $\underline{\underline{\sigma}}=\underline{\underline{A}} \underline{\underline{\varepsilon}}(\underline{u})$ by $\underline{\underline{A}}^{-1} \underline{\underline{\sigma}}=\nabla \underline{u}-\underline{\gamma}(\underline{u})$, using the relation $\underline{\underline{\varepsilon}}(\underline{u})=\nabla \underline{u}-\underline{\gamma}(\underline{u})$, where $\underline{\underline{\gamma}}(\underline{u})=\frac{1}{2}\left[\begin{array}{cc}0 & \partial_{2} u_{1}-\partial_{1} u_{2} \\ \partial_{1} u_{2}-\partial_{2} u_{1} & 0\end{array}\right]$. A new equation $\underline{\underline{\sigma}}-\underline{\underline{\sigma}}^{T}=0$ enforces the desired stress symmetry, and we introduce the rotation variable $q=\frac{1}{2} \operatorname{asym} \nabla \underline{u}$, where $\operatorname{asym} \underline{\underline{\tau}}=\tau_{12}-\tau_{21}$ is the asymmetry measure defined for tensors $\underline{\underline{\tau}}=\left[\begin{array}{ll}\tau_{11} & \tau_{12} \\ \tau_{21} & \tau_{22}\end{array}\right]$. 
Under this point of view, and adopting the simplified notation $\mathscr{S}=H(\operatorname{div}, \Omega, \mathbb{M}), \mathscr{U}=L^{2}\left(\Omega, \mathbb{R}^{2}\right)$, and $\mathscr{Q}=L^{2}(\Omega)$, the mixed formulation with weakly imposed stress symmetry searches for $(\underline{\underline{\sigma}}, \underline{u}, q) \in \mathcal{E}:=\mathscr{S} \times \mathscr{U} \times \mathscr{Q}$ satisfying

$$
\begin{aligned}
\left(\underline{\underline{A}}^{-1} \underline{\underline{\sigma}}, \underline{\underline{\tau}}\right)+(\underline{u}, \underline{\nabla} \cdot \underline{\underline{\tau}})+(q, \operatorname{asym} \underline{\underline{\tau}}) & =\left\langle\underline{\underline{\tau}} \underline{\underline{n}}^{\Omega}, \underline{g}\right\rangle, & & \forall \underline{\underline{\tau}} \in \mathscr{S}, \\
-(\underline{\nabla} \cdot \underline{\underline{\sigma}}, \underline{v}) & =(\underline{f}, \underline{v}), & & \forall \underline{v} \in \mathscr{U}, \\
(\operatorname{asym} \underline{\underline{\sigma}}, w) & =0, & & \forall w \in \mathscr{Q} .
\end{aligned}
$$

This kind of method belongs to a classical methodology. It dates from the seventies, in the pioneering period of mathematical analysis for mixed and hybrid formulations. They typically appear in minimization problems with constraints $(e . g .$, see $[7,8,38])$. In this formulation, there are two constraints. The first one is for the realization of the divergence equation (2.3), and displacement plays the role of the corresponding Lagrange multiplier. The other multiplier is $q$, used for the weak enforcement of stress symmetry in (2.4).

\subsection{Hybrid local-global version}

The purpose of the hybrid local-global version of the stress mixed formulation (2.2)-(2.4) is to naturally derive stable bases for the two-level discrete method in Section 3. For that, we define a partition $\mathcal{T}=\left\{\Omega_{i}\right\}$ of the domain $\Omega$. Associated to $\mathcal{T}$, let $\Gamma$ be the mesh skeleton formed by the union of the boundaries $\partial \Omega_{i}$. To make inter-element connections, we introduce the multiplier $\underline{\lambda}$ which lives in a normal trace space defined over $\Gamma$ :

$$
\Lambda:=\Lambda\left(\Gamma, \mathbb{R}^{2}\right)=\left\{\underline{\mu} ; \underline{\mu}=\left.\underline{\underline{\tau}} \underline{\underline{n}}\right|_{\partial \Omega_{i}}, \underline{\underline{\tau}} \in H(\operatorname{div}, \Omega, \mathbb{M}), \Omega_{i} \in \mathcal{T}\right\},
$$

where $\underline{n}$ is a given vector field defined over $\Gamma$ and normal to $\partial \Omega_{i}$. Notice that $\left.\underline{n}\right|_{\Omega_{i}}=\delta_{i} \underline{n}^{\Omega_{i}}$, where $\delta_{i}(e)=\left.\underline{n} \cdot \underline{n}^{\Omega_{i}}\right|_{e}$ for all edges $e \subset \partial \Omega_{i}$ (i.e., $\delta_{i}(e)=1$ for boundary edges and $\delta_{i}(e)=-\delta_{j}(e)$ for interface edges $e \subset \Omega_{i} \cap \Omega_{j}$ ). The displacement is decomposed as $\underline{u}=\underline{u}_{r m}+\underline{u}^{\perp}$, where $\underline{u}_{r m} \in \mathscr{U}_{r m}$ is a piecewise rigid body mode over $\mathcal{T}$. Precisely,

$$
\mathscr{U}_{r m}:=\left\{\underline{u} \in \mathscr{U} ; \underline{u}_{i}=\left.\underline{u}\right|_{\Omega_{i}} \in \mathscr{U}_{r m}\left(\Omega_{i}\right), \Omega_{i} \in \mathcal{T}\right\}, \quad \mathscr{U}_{r m}\left(\Omega_{i}\right):=\left\{(\alpha, \beta)+\rho(-y, x) ;(x, y) \in \Omega_{i}, \alpha, \beta, \rho \in \mathbb{R}\right\} .
$$

The complementary displacement term $\underline{u}^{\perp}$ lives in $\mathscr{U}^{\perp} \subset \mathscr{U}$, the $L^{2}$-orthogonal complement of $\mathscr{U}_{r m}$. For local Neumann problems, test tensors should be bubble functions in $\mathscr{\mathscr { S }}\left(\Omega_{i}\right)=\left\{\underline{\underline{\tau}} \in \mathscr{S} ;\left.\underline{\underline{\tau}} \underline{\underline{n}}\right|_{\partial \Omega_{i}}=0\right\}$.

The hybrid version is formulated in two stages. There is a global system of equations for the multiplier $\lambda \in \Lambda$ and the rigid body motion displacement component $\underline{u}_{r m} \in \mathscr{U}_{r m}$. To obtain the solution $(\underline{\sigma}, \underline{u}, q) \in \mathscr{S} \times \mathscr{U} \times \mathscr{Q}$, we add local fine scale components to the solution of the global stage as follows

$$
\underline{\underline{\sigma}}=T^{\underline{\sigma}}(\underline{\lambda})+\hat{T}^{\underline{\sigma}}(\underline{f}), \quad \underline{u}=\underline{u}_{r m}+T^{\underline{u}}(\underline{\lambda})+\hat{T}^{\underline{u}}(\underline{f}), \quad q=\frac{1}{2} \operatorname{asym} \nabla \underline{u}_{r m}+T^{q}(\underline{\lambda})+\hat{T}^{q}(\underline{f}),
$$

where

$$
T: \Lambda \rightarrow \mathscr{S} \times \mathscr{U}^{\perp} \times \mathscr{Q}, \quad \hat{T}: \mathscr{U} \rightarrow \mathscr{S} \times \mathscr{U}^{\perp} \times \mathscr{Q},
$$

are linear operators whose images are solutions of local Neumann elasticity problems on each subregion $\Omega_{i}$. Based on the above functional framework, consider the local-global stages:

- Local stage: For $\underline{\lambda} \in \Lambda, \underline{\lambda} \neq 0$, and $f \in L^{2}\left(\Omega, \mathbb{R}^{2}\right)$, let the operators $T(\underline{\lambda})=\left\{T^{\underline{\sigma}}(\underline{\lambda}), T^{\underline{u}}(\underline{\lambda}), T^{q}(\underline{\lambda})\right\}$ and $\hat{T}(\underline{f})=\left\{\hat{T}^{\underline{\sigma}}(\underline{f}), \hat{T}^{\underline{u}}(\underline{f}), \hat{T}^{q}(\underline{f})\right\}$ be locally defined in each $\Omega_{i}$ by the following mixed formulations with weakly 
imposed stress symmetry and Neumann boundary conditions:

$$
\begin{aligned}
& \left(\underline{\nabla} \cdot T^{\underline{\sigma}}(\underline{\lambda}), \underline{v}\right)_{\Omega_{i}}=0, \\
& \forall \underline{v} \in \mathscr{U}^{\perp}\left(\Omega_{i}\right), \\
& \left(\underline{\underline{A}}^{-1} T^{\underline{\sigma}}(\underline{\lambda}), \underline{\underline{\tau}}\right)_{\Omega_{i}}+\left(T^{\underline{u}}(\underline{\lambda}), \underline{\nabla} \cdot \underline{\underline{\tau}}\right)_{\Omega_{i}}+\left(T^{q}(\underline{\lambda}), \operatorname{asym} \underline{\underline{\tau}}\right)_{\Omega_{i}}=0, \quad \forall \underline{\underline{\tau}} \in \dot{\mathscr{S}}\left(\Omega_{i}\right), \\
& (\operatorname{asym} T \stackrel{\sigma}{\stackrel{(}{\lambda}}(\underline{\lambda}), \varphi)_{\Omega_{i}}=0, \quad \forall \varphi \in L^{2}\left(\Omega_{i}\right), \\
& \left.T \stackrel{\underline{\sigma}}{(\underline{\lambda})} \underline{n}\right|_{\partial \Omega_{i}}=\left.\underline{\lambda}\right|_{\partial \Omega_{i}} \text {. } \\
& -(\underline{\nabla} \cdot \hat{T} \underline{\underline{\sigma}}(\underline{f}), \underline{v})_{\Omega_{i}}=(\underline{f}, \underline{v})_{\Omega_{i}}, \quad \forall \underline{v} \in \mathscr{U}^{\perp}\left(\Omega_{i}\right), \\
& \left(\underline{\underline{A}}^{-1} \hat{T}^{\underline{\underline{\sigma}}}(\underline{f}), \underline{\underline{\tau}}\right)_{\Omega_{i}}+\left(\hat{T}^{\underline{u}}(\underline{f}), \underline{\nabla} \cdot \underline{\underline{\tau}}\right)_{\Omega_{i}}+\left(\hat{T}^{q}(\underline{f}), \operatorname{asym} \underline{\underline{\tau}}\right)_{\Omega_{i}}=0, \quad \forall \underline{\underline{\tau}} \in \stackrel{\mathscr{S}}{=}\left(\Omega_{i}\right), \\
& \left(\operatorname{asym} \hat{T}^{\underline{\sigma}}(\underline{f}), \varphi\right)_{\Omega_{i}}=0, \quad \forall \varphi \in L^{2}\left(\Omega_{i}\right) \text {, } \\
& \left.\hat{T}^{\underline{\sigma}}(\underline{f}) \underline{n}\right|_{\partial \Omega_{i}}=0 .
\end{aligned}
$$

- Global stage: Given $\underline{f} \in \mathscr{U}$, and $\underline{g} \in H^{\frac{1}{2}}\left(\partial \Omega, \mathbb{R}^{2}\right)$, find $\underline{u}_{r m} \in \mathscr{U}_{r m}$ and $\underline{\lambda} \in \Lambda$ that solve

$$
\begin{aligned}
\left(\underline{\underline{A}}^{-1} T^{\underline{\underline{\sigma}}}(\underline{\lambda}), T^{\underline{\sigma}}(\underline{\mu})\right)+ & \left(\underline{u}_{r m}, \underline{\nabla} \cdot T^{\underline{\sigma}}(\underline{\mu})\right)=-\left(\underline{f}, T^{\underline{u}}(\underline{\mu})\right)+\langle\underline{\mu}, \underline{g}\rangle, & & \forall \underline{\mu} \in \Lambda, \\
& -(\underline{\nabla} \cdot T \underline{\underline{\sigma}}(\underline{\lambda}), \underline{v})=(\underline{f}, \underline{v}), & & \forall \underline{v} \in \mathscr{U}_{r m} .
\end{aligned}
$$

Notice that the boundary data for $T(\underline{\lambda})$ is $\underline{\lambda}$, while vanishing Neumann boundary conditions are applied for $\hat{T}(\underline{f})$. Knowing $\underline{\lambda}$, these operators define the stress $\underline{\underline{\sigma}} \in \mathscr{S}$, the fine scale rigid-body-motion-free component $\underline{u}^{\perp}:=T^{\underline{u}}(\underline{\lambda})+\hat{T}^{\underline{u}}(\underline{f}) \in \mathscr{U}^{\perp}$, and the part $T^{q}(\underline{\lambda})+\underline{\overline{\hat{T}}}^{q}(f)$ required to form $q$. The missing information comes from the global system to be solved for $\underline{u}_{r m}$ and $\underline{\lambda}$. The following theorem states the well-posedness of the local-global continuous formulation (2.7)-(2.16). This formulation naturally derives multiscale discrete versions, as discussed in the following sections.

Theorem 2.1. The global system (2.15) and (2.16) has a unique solution $\left(\underline{u}_{r m}, \underline{\lambda}\right) \in \mathscr{U}_{r m} \times \Lambda$. Moreover, a function $(\underline{\underline{\sigma}}, \underline{u}, q)$ is recovered from $\left(\underline{u}_{r m}, \underline{\lambda}\right)$ as stated in $(2.5)$, by solving the local problems $T(\lambda)$, defined in $(2.7)-(2.10)$ and $\hat{T}(\underline{f})$, defined in (2.11)-(2.14), if and only if $(\underline{\underline{\sigma}}, \underline{u}, q)$ solves the weak formulation (2.2)-(2.4).

Proof. It is postponed to Appendix A.1.

\section{Remarks}

The hybrid local-global characterization given in Theorem 2.1 modifies the one for the MHM- $H^{1}$ method proposed in [28]. The MHM- $H^{1}$ local-global characterization of the exact solution is based on the classical primal hybrid approach for the displacement formulation of the elasticity problem. The fine scale information incorporated into the global system in this primal approach comes from a different kind of local solver. Instead of using local stress mixed formulations with weak symmetry in each subregion $\Omega_{i}$, the second stage of the primal MHM formulation solves local Neumann elasticity problems on the displacement field only. Differently from the current work, the stress field in the MHM- $H^{1}$ methods are not in the desirable $H(\operatorname{div}, \Omega, \mathbb{M})$ and there is so far no result that assures the convergence of the divergence of the stress.

\section{DisCRETE TWO-SCALE STRESS HYBRID-MIXED MODELS WITH REDUCED SYMMETRY}

This section is dedicated to discrete elasticity models derived from the stress mixed formulation with reduced stress symmetry and based on two-scale FE space settings $\mathcal{E}_{\gamma}$. Our main goal is to propose and analyze the 

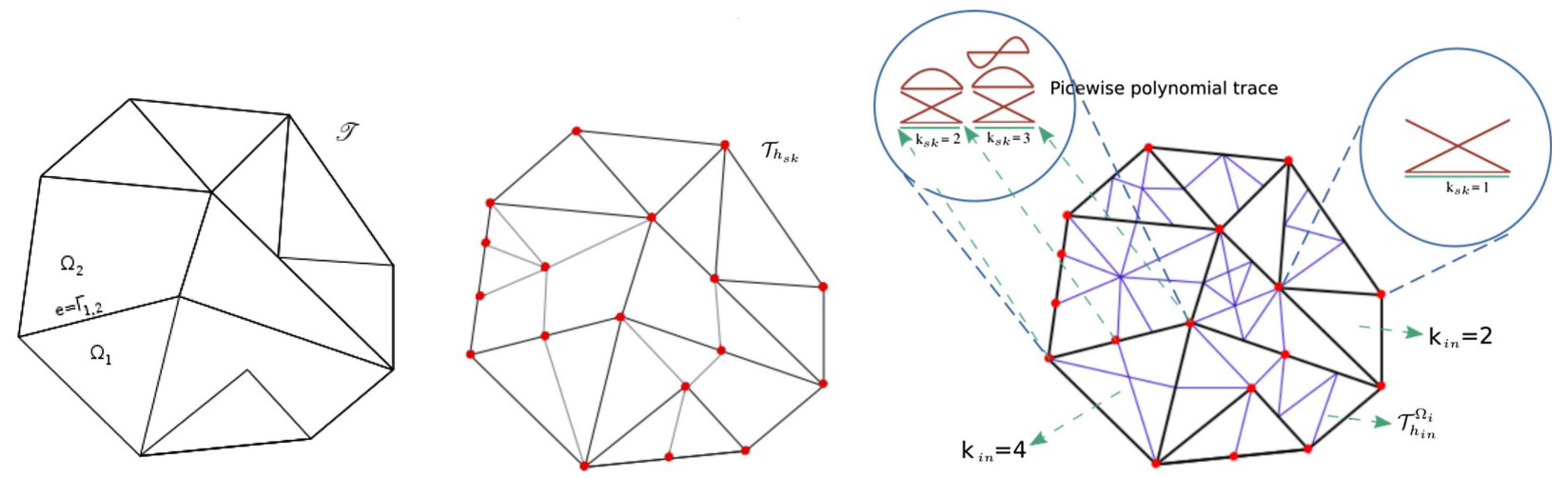

FIGURE 1. Diagram illustration of some aspects of a hierarchy of partitions and discretization parameters: a macro-partition $\mathcal{T}$ (left-image), coarsest conformal mesh $\mathcal{T}_{h_{s k}}$ (center-image), refined partitions $\mathcal{T}_{h_{i n}}^{\Omega_{i}}$ and polynomial degrees $k_{s k}$ and $k_{i n}$ (right-image).

method denoted by the acronym $\operatorname{MHM}-\mathrm{WS}\left(\mathcal{E}_{\gamma}\right)$, following the principles of the local-global hybrid characterization described in Section 2.2. For that, we present a unified and flexible procedure for the construction of hierarchies of two-scale meshes supporting the FE spaces $\mathcal{E}_{\gamma}$. We define two sets of parameters $\gamma:=\left(\gamma_{s k}, \gamma_{i n}\right)$, where $\gamma_{s k}=\left(h_{s k}, k_{s k}\right)$ and $\gamma_{i n}=\left(h_{i n}, k_{i n}\right)$ are used to indicate the mesh widths and polynomial degrees of two scales: coarse and fine. The focus is on two-scale frameworks, but single-level cases $\gamma_{s k}=\gamma_{i n}$ may be treated in the same context as well.

\subsection{Two-scale partitions and finite element spaces}

The two-scale mesh and FE space hierarchies are obtained by the following stages.

\subsubsection{Discretization parameters and mesh hierarchy}

- Given $\gamma_{s k}=\left(h_{s k}, k_{s k}\right)$, let $\mathcal{T}_{h_{s k}}$ be a conformal shape regular partition of $\Omega$ formed by the union of sub-meshes $\mathcal{T}_{h_{s k}}^{\Omega_{i}}=\{K\}$, all of them with characteristic size $h_{s k}$.

- Refined internal partitions $\mathcal{T}_{h_{i n}}^{\Omega_{i}}$ are obtained by the subdivision of $\mathcal{T}_{h_{s k}}^{\Omega_{i}}$. We choose the mesh characteristic size $h_{i n}$ such that $h_{i n} \sim h_{s k} / 2^{\ell}$, for a given integer $\ell \geq 0$. Define $\gamma_{i n}=\left(h_{i n}, k_{i n}\right)$, where $k_{i n}=k_{s k}+n$, for a given integer $n \geq 0$.

- Define the partition $\mathcal{T}^{\Gamma}=\{F\}$ of $\Gamma$ by taking the edges $F$ induced by $\mathcal{T}_{h_{s k}}$ over $\Gamma \backslash \partial \Omega$, and the edges $F$ induced by $\mathcal{T}_{h_{i n}}^{\Omega_{i}}$ over $\partial \Omega \cap \partial \Omega_{i}$. Thus, the characteristic sizes are $h_{s k}$ for internal edges, and $h_{i n}$ otherwise.

Figure 1 illustrates some aspects of the two-scale hierarchy of meshes: the macro-partition $\mathcal{T}$, a conformal partition $\mathcal{T}_{h_{s k}}$, and local refined partitions $\mathcal{T}_{h_{i n}}^{\Omega_{i}}$. Observe that, over an edge $e=\Gamma_{i, j}=\Omega_{i} \cap \Omega_{j}$, the meshes $\mathcal{T}_{h_{i n}}^{\Omega_{i}}$ and $\mathcal{T}_{h_{i n}}^{\Omega_{j}}$ do not necessarily need to be conformal (e.g., in $e=\Gamma_{1,2}$ ). We also show examples of polynomial degrees over the subregions and over edges of the mesh skeleton. Although $\gamma_{s k}$ and $\gamma_{i n}$ do not need to be uniform, as illustrated in Figure 1, for simplicity we shall only consider uniform distributions for them.

\subsubsection{Two-scale FE space settings}

- Let $\Lambda_{\gamma} \subset \Lambda$ be the trace space piecewisely defined over $\mathcal{T}^{\Gamma}$ by polynomials of degree $\leq k_{s k}$ over the internal edges, and of degree $k_{i n}$ over the boundary edges. 
- Define refined single-level FE spaces in the interior of the subregions on top of the internal partitions $\mathcal{T}_{h_{i n}}^{\Omega_{i}}$

$$
\begin{aligned}
& \mathscr{S}_{\gamma_{i n}}\left(\Omega_{i}\right)=\left\{\underline{\underline{\tau}} \in H\left(\operatorname{div}, \Omega_{i}, \mathbb{M}\right) ;\left.\underline{\underline{\tau}}\right|_{K} \in S(K, \mathbb{M}), \forall K \in \mathcal{T}_{h_{i n}}^{\Omega_{i}}\right\}, \\
& \mathscr{U}_{\gamma_{i n}}\left(\Omega_{i}\right)=\left\{\underline{u} \in L^{2}\left(\Omega_{i}, \mathbb{R}^{2}\right) ;\left.\underline{u}\right|_{K} \in U\left(K, \mathbb{R}^{2}\right), \forall K \in \mathcal{T}_{h_{i n}}^{\Omega_{i}}\right\}, \\
& \mathscr{Q}_{\gamma_{i n}}\left(\Omega_{i}\right)=\left\{q \in L^{2}\left(\Omega_{i}\right) ;\left.q\right|_{K} \in Q(K), \forall K \in \mathcal{T}_{h_{i n}}^{\Omega_{i}}\right\},
\end{aligned}
$$

in terms of local FE spaces $S(K, \mathbb{M}), U\left(K, \mathbb{R}^{2}\right)$ and $Q(K)$ for the elements $K \in \mathcal{T}_{h_{i n}}^{\Omega_{i}}$. The degree $k_{i n}$ refers to the polynomials associated to the normal traces over $\partial K$ of the tensors $\underline{\underline{\tau}} \in S(K, \mathbb{M})$.

- In this setting, we introduce the two-scale tensor FE spaces $\mathscr{S}_{\gamma}\left(\Omega_{i}\right)$, composed by functions in $\mathscr{S}_{\gamma_{i n}}\left(\Omega_{i}\right)$ whose normal traces are constrained to $\Lambda_{\gamma}$, i.e.,

$$
\mathscr{S}_{\gamma}\left(\Omega_{i}\right)=\left\{\underline{\underline{\tau}} \in \mathscr{S}_{\gamma_{i n}}\left(\Omega_{i}\right) ;\left.\left.\underline{\underline{\tau}} \underline{\underline{n}}\right|_{\partial \Omega_{i} \backslash \partial \Omega} \in \Lambda_{\gamma}\right|_{\partial \Omega_{i} \backslash \partial \Omega}\right\}
$$

Notice that the constrained tensor space (3.4) is well defined due to the fact the trace functions induced by $\mathscr{S}_{\gamma_{i n}}\left(\Omega_{i}\right)$ over $\partial \Omega_{i}$ are piecewisely defined by polynomials of degree $k_{i n} \geq k_{s k}$ on top of elements obtained by the refinement of the mesh $\mathcal{T}^{\Gamma} \cap \partial \Omega_{i}$. Moreover, $\mathscr{S}_{\gamma}\left(\Omega_{i}\right)$ can be expressed as a two-scale direct sum $\mathscr{S}_{\gamma}\left(\Omega_{i}\right)=\mathscr{S}_{\gamma}^{\partial}\left(\Omega_{i}\right) \oplus \dot{\mathscr{S}}_{\gamma_{i n}}\left(\Omega_{i}\right)$, where $\dot{\mathscr{S}}_{\gamma_{i n}}\left(\Omega_{i}\right)$ is the set of bubble tensors, with vanishing normal traces over $\partial \Omega_{i}$, having refined resolution. The edge tensors in $\mathscr{S}_{\gamma}^{\partial}\left(\Omega_{i}\right)$ have normal traces over $\partial \Omega_{i} \backslash \partial \Omega$ constrained to $\Lambda_{\gamma}$ having coarser resolution $\gamma_{s k}$ over internal edges $F \in \mathcal{T}^{\Gamma}$.

- Finally, let $\mathcal{E}_{\gamma}=\mathscr{S}_{\gamma} \times \mathscr{U}_{\gamma_{i n}} \times \mathscr{Q}_{\gamma_{i n}} \subset H(\operatorname{div}, \Omega, \mathbb{M}) \times L^{2}\left(\Omega, \mathbb{R}^{2}\right) \times L^{2}(\Omega)$ be the two-scale FE space whose restriction to each subdomain $\Omega_{i} \in \mathcal{T}$ is the local FE space $\mathcal{E}_{\gamma}\left(\Omega_{i}\right)=\mathscr{S}_{\gamma}\left(\Omega_{i}\right) \times \mathscr{U}_{\gamma_{i n}}\left(\Omega_{i}\right) \times \mathscr{Q}_{\gamma_{i n}}\left(\Omega_{i}\right)$. We restrict the analysis to displacement FE spaces satisfying $\mathscr{U}_{r m} \subset \mathscr{U}_{\gamma_{i n}}$, and let $\mathscr{U}_{\gamma_{i n}}^{\perp}$ be the $L^{2}$-orthogonal complement of $\mathscr{U}_{r m}$ in $\mathscr{U}_{\gamma_{i n}}$, with local components $\mathscr{U}_{\gamma_{i n}}^{\perp}\left(\Omega_{i}\right)$.

\subsection{Derivation of the MHM-WS $\left(\mathcal{E}_{\gamma}\right)$ method}

The two discrete local-global building blocks of information-passing in the MHM-WS $\left(\mathcal{E}_{\gamma}\right)$ scheme shall be referred as downscaling and upscaling stages, following a terminology usually used in multiscale contexts (see e.g., [21]). At the coarsest scale level, $\underline{\tilde{\lambda}} \in \Lambda_{\gamma}$ and $\underline{\tilde{u}}_{r m} \in \mathscr{U}_{r m}$ are computed by a global system (upscaling stage). At the fine scale level, $\underline{\tilde{u}}^{\perp}:=\tilde{T}^{\underline{u}}(\underline{\tilde{\lambda}})+\tilde{\hat{T}}^{\underline{u}}(\underline{f}) \in \mathscr{U}_{\gamma_{i n}}^{\perp}, \underline{\underline{\tilde{\sigma}}} \in \mathscr{S}_{\gamma}$ and $\tilde{q} \in \mathscr{Q}_{\gamma_{i n}}$, are solutions of a set of completely independent Neumann boundary local problems restricted to the subregions $\Omega_{i} \in \mathcal{T}$ (downscaling stage). These local systems may be represented by the action of operators $\tilde{T}: \Lambda_{\gamma} \rightarrow \mathscr{S}_{\gamma} \times \mathscr{U}_{\gamma_{i n}}^{\perp} \times \mathscr{Q}_{\gamma_{i n}}$ and $\tilde{\hat{T}}: \mathscr{U} \rightarrow \mathscr{S}_{\gamma} \times \mathscr{U}_{\gamma_{i n}}^{\perp} \times \mathscr{Q}_{\gamma_{i n}}$, as discrete versions of the operators (2.6) defined in Section 2.2.

Using this procedure we characterize the approximate solution as a discrete counterpart of (2.5):

$$
\underline{\underline{\tilde{\sigma}}}=\tilde{T}=\underline{\underline{\sigma}}(\underline{\tilde{\lambda}})+\tilde{\hat{T}} \underline{\underline{\sigma}}(\underline{f}), \quad \underline{\tilde{u}}=\underline{\tilde{u}}_{r m}+\tilde{T}^{\underline{u}}(\underline{\tilde{\lambda}})+\tilde{\hat{T}}^{\underline{u}}(\underline{f}), \quad \tilde{q}=\frac{1}{2} \operatorname{asym} \nabla \underline{\tilde{u}}_{r m}+\tilde{T}^{q}(\underline{\tilde{\lambda}})+\tilde{\hat{T}}^{q}(\underline{f}) .
$$

Precisely, the local-global discrete systems composing the MHM-WS $\left(\mathcal{E}_{\gamma}\right)$ scheme, transferring information from the fine to the coarse-scale level and vice versa, are written in the following form.

- Local stage (Downscaling): For $\underline{\tilde{\lambda}} \in \Lambda_{\gamma}, \underline{\tilde{\lambda}} \neq 0$, and $\underline{f} \in L^{2}\left(\Omega, \mathbb{R}^{2}\right)$, let the operators $\tilde{T}(\underline{\tilde{\lambda}})=$ $\left\{\tilde{T} \underline{\underline{\sigma}}(\underline{\tilde{\lambda}}), \tilde{T}^{\underline{u}}(\underline{\tilde{\lambda}}), \tilde{T}^{q}(\underline{\lambda})\right\}$ and $\tilde{\hat{T}}(\underline{f})=\left\{\tilde{\hat{T}}^{\underline{\sigma}}(\underline{f}), \tilde{\hat{T}}^{\underline{u}}(\underline{f}), \tilde{\hat{T}}^{q}(\underline{f})\right\}$ be determined in each subregion $\Omega_{i}$ by the follow- 
ing $\operatorname{MFEM-WS}\left(\mathcal{E}_{\gamma}\right)$ formulations locally defined in each $\Omega_{i}$ with Neumann boundary conditions:

$$
\begin{aligned}
& \left(\underline{\nabla} \cdot \tilde{T}^{\underline{\sigma}}(\underline{\tilde{\lambda}}), \underline{v}\right)_{\Omega_{i}}=0, \quad \forall \underline{v} \in \mathscr{U}_{\gamma_{i n}}^{\perp}\left(\Omega_{i}\right), \\
& \left(\underline{\underline{A}}^{-1} \tilde{T}^{\underline{\sigma}}(\underline{\tilde{\lambda}}), \underline{\underline{\tau}}\right)_{\Omega_{i}}+\left(\tilde{T}^{\underline{u}}(\underline{\tilde{\lambda}}), \underline{\nabla} \cdot \underline{\underline{\tau}}\right)_{\Omega_{i}}+\left(\tilde{T}^{q}(\underline{\tilde{\lambda}}), \operatorname{asym} \underline{\underline{\tau}}\right)_{\Omega_{i}}=0, \quad \forall \underline{\underline{\tau}} \in \dot{\mathscr{S}}_{\gamma}\left(\Omega_{i}\right), \\
& \left(\operatorname{asym} \tilde{T}^{\underline{\sigma}}(\underline{\tilde{\lambda}}), \varphi\right)_{\Omega_{i}}=0, \quad \forall \varphi \in \mathscr{Q}_{\gamma_{i n}}\left(\Omega_{i}\right), \\
& \left.\tilde{T} \stackrel{\sigma}{\underline{\sigma}}(\underline{\tilde{\lambda}}) \underline{n}\right|_{\partial \Omega_{i}}=\left.\underline{\tilde{\lambda}}\right|_{\partial \Omega_{i}} . \\
& -(\underline{\nabla} \cdot \tilde{\hat{T}} \underline{\underline{\sigma}}(\underline{f}), \underline{v})_{\Omega_{i}}=(\underline{f}, \underline{v})_{\Omega_{i}}, \quad \forall \underline{v} \in \mathscr{U}_{\gamma_{i n}}^{\perp}\left(\Omega_{i}\right), \\
& \left(\underline{\underline{A}}^{-1} \tilde{\hat{T}}^{\underline{\sigma}}(\underline{f}), \underline{\underline{\tau}}\right)_{\Omega_{i}}+(\tilde{\hat{\hat{T}}} \underline{\underline{u}}(\underline{f}), \underline{\nabla} \cdot \underline{\underline{\tau}})_{\Omega_{i}}+\left(\tilde{\hat{T}}^{q}(\underline{f}), \operatorname{asym} \underline{\underline{\tau}}\right)_{\Omega_{i}}=0, \quad \forall \underline{\underline{\tau}} \in \dot{\mathscr{S}}_{\gamma}\left(\Omega_{i}\right), \\
& (\operatorname{asym} \tilde{\hat{T}} \stackrel{\sigma}{=}(\underline{f}), \varphi)_{\Omega_{i}}=0, \quad \forall \varphi \in \mathscr{Q}_{\gamma_{i n}}\left(\Omega_{i}\right), \\
& \left.\tilde{\hat{T}} \stackrel{\sigma}{=}(\underline{f}) \underline{n}\right|_{\partial \Omega_{i}}=0 .
\end{aligned}
$$

- Global stage (Upscaling): $\underline{\tilde{u}}_{r m} \in \mathscr{U}_{r m}$ and $\underline{\tilde{\lambda}} \in \Lambda_{\gamma}$ are determined by the global system

$$
\begin{aligned}
\left(\underline{\underline{A}}^{-1} \tilde{T}^{\underline{\sigma}}(\underline{\tilde{\lambda}}), \tilde{T}^{\underline{\sigma}}(\underline{\mu})\right)+ & \left(\underline{\tilde{u}}_{r m}, \underline{\nabla} \cdot \tilde{T}^{\underline{\underline{\sigma}}}(\underline{\mu})\right)=-\left(\underline{f}, \tilde{T}^{\underline{u}}(\underline{\mu})\right)+\langle\underline{\mu}, \underline{g}\rangle, & & \forall \underline{\mu} \in \Lambda_{\gamma}, \\
& -\left(\underline{\nabla} \cdot \tilde{T}^{\underline{\sigma}}(\underline{\tilde{\lambda}}), \underline{v}\right)=(\underline{f}, \underline{v}), & & \forall \underline{v} \in \mathscr{U}_{r m} .
\end{aligned}
$$

\subsection{MHM-WS $\left(\mathcal{E}_{\gamma}\right)$ as a MFEM-WS $\left(\mathcal{E}_{\gamma}\right)$ formulation}

As for the weak formulations in infinite dimension, a remarkable property of the MHM-WS( $\left.\mathcal{E}_{\gamma}\right)$ method is that it can be interpreted as a hybrid local-global characterization of a discrete stress mixed formulation with reduced symmetry based on the FE space setting $\mathcal{E}_{\gamma}=\mathscr{S}_{\gamma} \times \mathscr{U}_{\gamma_{i n}} \times \mathscr{Q}_{\gamma_{i n}}$. It is denoted here by the acronym $\operatorname{MFEM}-\mathrm{WS}\left(\mathcal{E}_{\gamma}\right)$, and will play a crucial role in the forthcoming sections for the analysis of the MHM-WS $\left(\mathcal{E}_{\gamma}\right)$ method.

The MFEM-WS $\left(\mathcal{E}_{\gamma}\right)$ method searches for approximations $(\underline{\underline{\tilde{\sigma}}}, \underline{\tilde{u}}, \tilde{q}) \in \mathcal{E}_{\gamma}=\mathscr{S}_{\gamma} \times \mathscr{U}_{\gamma_{\text {in }}} \times \mathscr{Q}_{\gamma_{\text {in }}}$ such that

$$
\begin{aligned}
\left(\underline{\underline{A}}^{-1} \underline{\underline{\sigma}}, \underline{\underline{\tau}}\right)+(\underline{\underline{u}}, \underline{\nabla} \cdot \underline{\underline{\tau}})+(\tilde{q}, \operatorname{asym} \underline{\underline{\tau}}) & =\left\langle\underline{\underline{\tau}} \underline{n}^{\Omega}, \underline{g}\right\rangle, & & \forall \underline{\underline{\tau}} \in \mathscr{S}_{\gamma} \\
-(\underline{\nabla} \cdot \underline{\underline{\sigma}}, \underline{v}) & =(\underline{f}, \underline{v}), & & \forall \underline{v} \in \mathscr{U}_{\gamma_{i n}}, \\
(\operatorname{asym} \underline{\underline{\tilde{\sigma}}}, w) & =0, & & \forall \varphi \in \mathscr{Q}_{\gamma_{i n}} .
\end{aligned}
$$

Notice that classical formulations associated to single-level FE spaces, with $\gamma_{s k}=\gamma_{i n}$, are particular cases of the MFEM-WS $\left(\mathcal{E}_{\gamma}\right)$ method. Even though the original stress mixed formulation (2.2)-(2.4), with reduced stress symmetry, is well posed, this may not be true for discrete versions (3.16)-(3.18). According to Brezzi's theory, the FE spaces of each field cannot be chosen independently one from the other, i.e., they should be compatible, meaning that some stability (inf-sup) conditions are mandatory. This topic shall be clarified in Section 4 for the specific two-scale settings $\mathcal{E}_{\gamma}$ under consideration.

Assuming the FE spaces $\mathcal{E}_{\gamma}=\mathscr{S}_{\gamma} \times \mathscr{U}_{\gamma_{i n}} \times \mathscr{Q}_{\gamma_{i n}}$ verify the stability constraints for the two-scale mixed FE formulation MFEM-WS( $\left.\mathcal{E}_{\gamma}\right)$, the goal is to show the equivalence of its unique solution with the solution reconstructed from the local-global output of the MHM-WS $\left(\mathcal{E}_{\gamma}\right)$ method. The proof of this property has two stages: (i) we prove uniqueness for the MFEM-WS $\left(\mathcal{E}_{\gamma}\right)$ method (Thm. 3.1) and (ii) we prove that the unique solution of the MFEM-WS $\left(\mathcal{E}_{\gamma}\right)$ method solves the local-global MHM-WS $\left(\mathcal{E}_{\gamma}\right)$ systems (this implies existence of solution for the MHM-WS method). Equivalence holds as consequence of the uniqueness property held by both methods (Thm. 3.2). 
Theorem 3.1. Assume the FE spaces $\mathcal{E}_{\gamma}=\mathscr{S}_{\gamma} \times \mathscr{U}_{\gamma_{i n}} \times \mathscr{Q}_{\gamma_{i n}}$ verify the stability constraints for the MFEM-

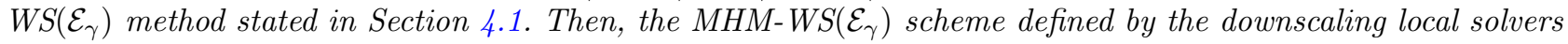
(3.6)-(3.9) and (3.10)-(3.13) and by the global upscaling system (3.14) and (3.15) has a unique solution.

Proof. The proof of this uniqueness result is postponed to Appendix A.2, and it makes use of the stability held by the $\operatorname{MFEM-WS}\left(\mathcal{E}_{\gamma}\right)$ formulation for the local Neumann problems.

The following theorem establishes the relation between the triad $(\underline{\underline{\tilde{\sigma}}}, \underline{\tilde{u}}, \tilde{q})$ recovered as in (3.5) from the approximate variables given by the $\operatorname{MHM}-\mathrm{WS}\left(\mathcal{E}_{\gamma}\right)$ scheme and the solution of the $\operatorname{MFEM}-\mathrm{WS}\left(\mathcal{E}_{\gamma}\right)$ formulation.

Theorem 3.2. Under the assumptions of Theorem 3.1, let $\left(\underline{\tilde{u}}_{r m}, \underline{\tilde{\lambda}}\right)$ be the unique solution of the MHM-WS( $\left.\mathcal{E}_{\gamma}\right)$ upscaling system (3.14) and (3.15). Then, $(\underline{\underline{\tilde{\sigma}}}, \underline{\tilde{u}}, \tilde{q})$ is the function recovered from $\left(\underline{\tilde{u}}_{r m}, \underline{\tilde{\lambda}}\right)$, as stated in $(3.5)$, by solving the local problems $\tilde{T}(\tilde{\lambda})$, defined in $(3.6)-(3.9)$, and $\tilde{\hat{T}}(\underline{f})$, defined in $(2.11)-(2.14)$, if and only if $(\underline{\underline{\tilde{\sigma}}}, \underline{\tilde{u}}, \tilde{q})$

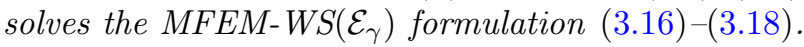

Proof. The proof of this equivalence result is documented in Appendix A.3.

Remarks

(i) By construction, the strong enforcement of the Neumann boundary conditions (3.9) and (3.13) is the reason to assume, from start, that $\underline{\underline{\underline{\sigma}}}=\tilde{T} \underline{\underline{\sigma}}(\underline{\tilde{\lambda}})+\tilde{\hat{T}}^{\underline{\sigma}}(f) \in \mathscr{S}_{\gamma}$, i.e., that the stress is globally $H(\operatorname{div})$-conforming. This is an important property of the $\operatorname{MHM}-\bar{W} S\left(\mathcal{E}_{\gamma}\right)$ solutions that, for instance, distinguish them from those of the multiscale mortar domain decomposition method [30].

(ii) The exercise of strongly enforcing the coarser traction variable in the constrained stress $\mathrm{FE}$ approximations of the MHM-WS $\left(\mathcal{E}_{\gamma}\right)$ scheme is a process that can be computationally accomplished in a similar manner as for conforming constrained functions commonly used in $h p$-adaptive strategies. Instead, the MHM- $H^{1}$ method in [28] imposes Neumann boundary conditions in a weak multiplier sense.

(iii) The approximate displacement $\underline{\tilde{u}}$ by the $\operatorname{MHM}-\mathrm{WS}\left(\mathcal{E}_{\gamma}\right)$ method decomposes as $\underline{\tilde{u}}=\underline{\tilde{u}}_{r m}+\underline{\tilde{u}}^{\perp}$, without continuity constraints for $\underline{u}^{\perp}:=\tilde{T}^{\underline{u}}(\underline{\tilde{\lambda}})+\tilde{\hat{T}}^{\underline{u}}(f)$ inside $\Omega_{i}$. This aspect, combined with the global $H(\operatorname{div})-$ conformity of the tensor $\underline{\underline{\sigma}}$, is crucial in the proof of the local conservation property verified by the MHM$\operatorname{WS}\left(\mathcal{E}_{\gamma}\right)$ method at the micro scale level. This is essential for ensuring local equilibrium. Furthermore, for $f=0$, the resulting tensor $\tilde{\tilde{\sigma}}$ is strongly divergence-free due to the divergence-compatibility condition (4.1) valid for $\mathscr{S}_{\gamma}\left(\Omega_{i}\right)$ and $\mathscr{U}_{\gamma_{i n}}\left(\overline{\bar{\Omega}}_{i}\right)$.

(iv) The local contribution $\tilde{\hat{T}}(f)$ of the numerical solution, defined in (3.10)-(3.13), is one of the important properties of the proposed multiscale method. Notably, such a perspective is paramount when $\underline{f}$ changes rapidly or embeds multiple scales. In particular, observe that if $\underline{f}$ belongs to $\mathscr{U}_{r m}$ then $\tilde{\hat{T}}(\underline{f})=0$. As a result, if $f$ is a low-degree polynomial function, then $\tilde{\hat{T}}(f)$ may be disregarded without undermining convergence. Importantly, such a contribution is local and then can be computed in parallel in the offline stage. Moreover, it does not impact the computational complexity of the method negatively, for its computation is local and "embarrassingly parallel" as the local problems are independent of one another. Finally, the contribution $\tilde{\hat{T}}(f)$ does not appear in the unknowns of the global system (3.14) and (3.15), for $\tilde{\hat{T}}^{\underline{\sigma}}(f)$ is a bubble function (with vanishing traction) and $\tilde{\hat{T}}^{\underline{u}}(f)$ is free of rigid body modes. As such, the solution of (3.10)-(3.13) can be entirely computed in the off-line stage, and then it does not enter in the most demanding computational effort for assembly and solve the global system (3.14) and (3.15). Also, the MHM methodology can handle problems with multi-query aspects, in which $\tilde{\hat{T}}(\underline{f})$ must be computed with several source terms $f$. In fact, one can adopt the strategy to compute $\tilde{\hat{T}}(\underline{\psi})$ first at the off-line stage, where $\psi$ stands for a function of a polynomial basis spanning an approximation of $\underline{f}$ without undermining convergence, and then reuse $\tilde{\hat{T}}(\underline{\psi})$ to calculate $\tilde{\hat{T}}(\underline{f})$ straightforwardly for all different $f$. 
(v) The relevant question about the robustness of the $\operatorname{MHM}-\mathrm{WS}\left(\mathcal{E}_{\gamma}\right)$ methods in terms of physical coefficients may be handled mathematically by the strategy proposed in [34] for the Poisson equation with oscillatory coefficients. In the sequel, we show numerical evidence in this regard and leave the subject's theoretical investigation to future works.

\section{Stability analysis for the MHM-WS $\left(\mathcal{E}_{\gamma}\right)$ Method}

In this section we give guidelines for effective construction of stable MHM-WS $\left(\mathcal{E}_{\gamma}\right)$ methods. In light of Theorem 3.2, stability is reached provided it is valid for the corresponding MFEM-WS $\left(\mathcal{E}_{\gamma}\right)$ methods. As such, let us first recall some standard stability requirements.

\subsection{Stability constraints}

As briefly remarked in the previous section, even though the stress mixed formulation with reduced symmetry $(2.2)-(2.4)$ is well posed, this may not be true for discrete versions of it, as for the MFEM-WS $\left(\mathcal{E}_{\gamma}\right)$ methods. According to Brezzis's theory, the FE spaces of each field can not be chosen independently one from the other, which must respect some compatibility conditions $[5,6,13,23]$. Following this theory, there are two steps in the path to derive stable two-scale elasticity triplets $\left\{\mathscr{S}_{\gamma}, \mathscr{U}_{\gamma_{i n}}, \mathscr{Q}_{\gamma_{i n}}\right\}$ for the relaxed-symmetry stress problem.

(1) To cope with the divergence constraint (3.17), the FE pair $\left\{\mathscr{S}_{\gamma}, \mathscr{U}_{\gamma_{i n}}\right\}$ for stress and displacement approximations should be divergence compatible

$$
\underline{\nabla} \cdot \mathscr{S}_{\gamma}=\mathscr{U}_{\gamma_{i n}} .
$$

Unfortunaltely, enforcing tensor $H$ (div)-conformity and the divergence constraint may, eventually, lead to complications for higher order schemes. One natural way to cope with (4.1) is to to built the rows and components of the two-scale pair $\mathscr{S}_{\gamma} \times \mathscr{U}_{\gamma_{i n}}$ by two-scale Poisson-compatible pair $\mathscr{V}_{\gamma} \times \mathscr{P}_{\gamma_{i n}}$ defined in [20], where $\mathscr{V}_{\gamma}=\mathscr{V}_{\gamma}^{\partial} \oplus \mathscr{\mathscr { V }}_{\gamma_{i n}}$ is a constrained two-scale flux space.

(2) Once divergence compatible stress and displacement FE spaces $\left\{\mathscr{S}_{\gamma}, \mathscr{U}_{\gamma_{i n}}\right\}$ are available, to complete the triplet $\left\{\mathscr{S}_{\gamma}, \mathscr{U}_{\gamma_{i n}}, \mathscr{Q}_{\gamma_{i n}}\right\}$ the next step is a stable choice of the FE space $\mathscr{Q}_{\gamma_{i n}}$. For two-dimensional problems, one option is to take $\mathscr{Q}_{\gamma_{i n}}$ from a Stokes-compatible FE pair $\left\{\mathscr{W}_{\gamma}, \mathscr{Q}_{\gamma_{i n}}\right\}$ such that the following Stokes constraint is satisfied:

$$
\underline{\underline{\nabla}} \times \mathscr{W}_{\gamma} \subset \mathscr{S}_{\gamma}
$$

In the sequel, we show how to accomplish this objective. We start with a given coarse single-level Stokescompatible pair $\left\{\mathscr{W}_{\gamma_{s k}}, \mathscr{Q}_{\gamma_{s k}}\right\}$ such that $\underline{\underline{\nabla}} \times \mathscr{W}_{\gamma_{s k}} \subset \mathscr{S}_{\gamma_{s k}}$. Using the methodology in [9], Stokes-consistency for a FE pair $\left\{\mathscr{W}_{\gamma}, \mathscr{Q}_{\gamma_{i n}}\right\}$ can be recovered when using the refined pressure space $\mathscr{Q}_{\gamma_{i n}}$ by enriching the velocity space $\mathscr{W}_{\gamma_{s k}}$ with some properly chosen bubble functions to form $\mathscr{W}_{\gamma}$. Moreover, we prove that the desired Stokes constraint (4.2) naturally holds.

In the sequel, some basic aspects of the required Poisson-compatible and Stokes-compatible FE pairs are clarified, including the examples that shall be adopted in the current work for the construction of stable FE settings $\mathcal{E}_{\gamma}$. The stability process shall be described with more details in Section 4.3 for these specific examples.

\subsection{Some stable FE pairs for Poisson and Stokes Problems}

For a triangular or quadrilateral element $K$, there is a reference element $\hat{K}$ and a geometric invertible map $F_{K}: \hat{K} \rightarrow K$ transforming $\hat{K}$ onto $K . F_{K}$ induces mappings $\mathbb{F}_{K}$ and $\mathbb{F}_{K}^{\text {div }}$ used to map functions defined in $\hat{K}$ to functions defined in $K$.

- Scalar functions: $p=\mathbb{F}_{K} \hat{p}=\hat{p} \circ F_{K}^{-1}$.

- Vector functions: $\underline{v}=\mathbb{F}_{K} \underline{\hat{v}}$, where $F_{K}$ is applied component-wisely. 
- Vector functions in $H\left(\operatorname{div}, K, \mathbb{R}^{2}\right): \underline{v}=\mathbb{F}_{K}^{\operatorname{div}} \underline{\hat{v}}=\mathbb{F}_{K}\left[\frac{1}{\mathbf{J}_{K}} \mathrm{D} F_{K} \underline{\hat{v}}\right]$, where $\mathrm{D} F_{K}$ is the Jacobian matrix of $F_{K}$, and $\mathbf{J}_{K}=\left|\operatorname{det}\left(\mathrm{D} F_{K}\right)\right|$ (Piola transformation).

- For tensors: $\underline{\underline{\tau}}=\mathbb{F}_{K}^{\text {div }} \underline{\underline{\hat{\tau}}}$ is the Piola transformation applied to each row of $\underline{\underline{\underline{\tau}}}$.

In $\hat{K}$, scalar polynomials are usually of the form: $\mathbb{P}_{k}(\hat{K})$, of total degree at most $k$, for the triangle; $\mathbb{Q}_{k, t}(\hat{K})$, of maximum degree $k$ in $x$ and $t$ in $y$, for the square.

\section{Poisson-compatible FE pairs}

Usually, the local FE spaces $V\left(K, \mathbb{R}^{2}\right)=\mathbb{F}_{K}^{\text {div }} \hat{\mathbf{V}}$ and $P(K)=\mathbb{F}_{K} \hat{P}$ are constructed by mapping polynomial spaces $\hat{\mathbf{V}}$ and $\hat{P}$ defined on a reference element $\hat{K}$. The stability (inf-sup) condition requires the divergenceconsistency condition $\nabla \cdot \hat{\mathbf{V}}=\hat{P}$. We consider that $\hat{\mathbf{V}}$ is spanned by a hierarchy of vector shape functions of two classes: functions of interior type in $\hat{\mathbf{V}}$, with vanishing normal traces over $\partial \hat{K}$, and functions associated to the element edges in $\hat{\mathbf{V}}^{\partial}$. Thus, the decomposition $\hat{\mathbf{V}}=\hat{\mathbf{V}}^{\partial} \oplus \stackrel{\hat{\mathbf{V}}}{\text { naturally holds. }}$

Divergence-consistency can be extended to the spaces $V\left(K, \mathbb{R}^{2}\right)$ and $P(K)$ by means of uniformly bounded interpolants $\boldsymbol{\pi}_{\gamma}^{D}: H^{1}\left(K, \mathbb{R}^{2}\right) \rightarrow V\left(K, \mathbb{R}^{2}\right)$ such that $\left(\nabla \cdot\left(\underline{\eta}-\boldsymbol{\pi}_{\gamma}^{D} \underline{\eta}\right), \psi\right)=0, \quad \forall \psi \in P(K)$. A general form to define $\boldsymbol{\pi}_{\gamma}^{D}$ is by the so called projection-based operators (see [16]). It is firstly defined in $\hat{K}$ using the representation $\boldsymbol{\pi}_{\gamma}^{D} \underline{\eta}=\boldsymbol{\pi}_{\gamma}^{D, \partial} \underline{\eta}+\stackrel{\circ}{\boldsymbol{\pi}}_{\gamma}^{D}\left(\underline{\eta}-\boldsymbol{\pi}_{\gamma}^{D, \partial} \underline{\eta}\right)$ in terms of edge and internal operators. Let $P(\partial \hat{K})$ be the space of normal traces of vector functions in $\hat{\mathbf{V}}$. For $\underline{\eta} \in H^{1}\left(\hat{K}, \mathbb{R}^{2}\right)$ and $\underline{\eta} \in H_{0}^{1}\left(\hat{K}, \mathbb{R}^{2}\right)$, the interpolants verify:

$$
\begin{aligned}
& \left\langle\boldsymbol{\pi}_{\gamma}^{D, \partial} \underline{\eta} \cdot \underline{n}^{\hat{K}}, \phi\right\rangle_{\partial \hat{K}}=\left\langle\underline{\eta} \cdot \underline{n}^{\hat{K}}, \phi\right\rangle_{\partial \hat{K}} ; \forall \phi \in P(\partial \hat{K}), \\
& \left(\nabla \cdot \stackrel{\circ}{\boldsymbol{\pi}}_{\gamma}^{D} \underline{\grave{\eta}}, \nabla \cdot \underline{w}\right)_{\hat{K}}=(\nabla \cdot \underline{i}, \nabla \cdot \underline{w})_{\hat{K}}, \quad \forall \underline{w} \in \dot{\hat{\mathbf{V}}}, \\
& \left(\stackrel{\circ}{\boldsymbol{\pi}}_{\gamma}^{D} \underline{\stackrel{\imath}{\eta}}, \underline{w}\right)_{\hat{K}}=(\underline{\circ}, \underline{w})_{\hat{K}}, \quad \forall \underline{w} \in \dot{\hat{\mathbf{V}}}, \nabla \cdot \underline{w}=0 .
\end{aligned}
$$

Then $\boldsymbol{\pi}_{\gamma}^{D}$ is extended to the computational elements $K$, and assembled to the whole domain $\Omega$.

In the current work, we shall deal with the following divergence-consistent FE pairs:

- For triangular elements: (Brezzi-Douglas-Marini FE pair and enriched versions):

- $\mathcal{B D} \mathcal{M}_{k}, k \geq 1[10]: \hat{V}_{\mathcal{B D} \mathcal{M}_{k}}=\mathbb{P}_{k}\left(\hat{K}, \mathbb{R}^{2}\right)$ and $P_{\mathcal{B D} \mathcal{M}_{k}}=\mathbb{P}_{k-1}(\hat{K})$.

- $\mathcal{B D} \mathcal{M}_{k}^{+}, k \geq 1[7]: \hat{V}_{\mathcal{B D M}_{k}^{+}}=\mathbb{P}_{k}^{\partial}\left(\hat{K}, \mathbb{R}^{2}\right) \oplus \stackrel{\stackrel{P}{P}}{k+1}_{k}\left(\hat{K}, \mathbb{R}^{2}\right), \hat{P}_{\mathcal{B D ~}_{k}^{+}}=\mathbb{P}_{k}(\hat{K})$ (known as BDF $\left.M_{k+1}\right)$.

- $\mathcal{B D}_{k}^{++}, k \geq 1[18]: \hat{V}_{\mathcal{B D} \mathcal{M}_{k}^{++}}^{+}=\mathbb{P}_{k}^{\partial}\left(\hat{K}, \mathbb{R}^{2}\right) \oplus \stackrel{\circ}{\mathbb{P}}_{k+2}\left(\hat{K}, \mathbb{R}^{2}\right), \hat{P}_{\mathcal{B D}_{k} \mathcal{M}_{k}^{++}}=\mathbb{P}_{k+1}(\hat{K})$.

- For quadrilateral elements (Raviart-Thomas FE pair and enriched version)

- $\mathcal{R} \mathcal{T}_{[k]}, k \geq 1[37]: \hat{V}_{\mathcal{R} \mathcal{T}_{[k]}}=\mathbb{Q}_{k+1, k}(\hat{K}) \times \mathbb{Q}_{k, k+1}(\hat{K})$ and $\hat{P}_{\mathcal{R} \mathcal{T}_{[k]}}=\mathbb{Q}_{k, k}(\hat{K})$.

- $\mathcal{R} \mathcal{T}_{[k]}^{+}, k \geq 1[18]: \hat{V}_{\mathcal{R} \mathcal{T}_{[k]}^{+}}=\hat{V}_{\mathcal{R} \mathcal{T}_{[k]}}^{\partial}(\hat{K}) \oplus \hat{\hat{V}}_{\mathcal{R} \mathcal{T}_{[k+1]}}(\hat{K})$ and $\hat{P}_{\mathcal{R} \mathcal{T}_{[k]}^{+}}=\mathbb{Q}_{k+1, k+1}(\hat{K})$.

Stokes-compatible FE pairs

The FE pairs used for velocity and pressure approximations in mixed Stokes formulations are generally defined by local finite element pairs $\left\{W\left(K, \mathbb{R}^{2}\right), Q(K)\right\}$. For stability, they should be compatible with the inf-sup condition. The following stable Stokes-compatible FE families shall be used in this paper:

- For triangular elements $K$ (Crouzeix-Raviart FE pair and enriched version):

- $\mathcal{C R}_{k}$, proposed in [14] for $k=2,3$, and extended to higher orders in [33]:

$W_{\mathcal{C R}_{k}}\left(K, \mathbb{R}^{2}\right)=\mathbb{P}_{k}\left(K, \mathbb{R}^{2}\right)+b_{K} \mathbb{P}_{k-2}\left(K, \mathbb{R}^{2}\right)$, where $b_{K}=\lambda_{1} \lambda_{2} \lambda_{3}$ are bubble functions defined by the barycentric coordinates $\lambda_{i}$ of $K$, and $Q_{\mathcal{C} \mathcal{R}_{k}}(K)=\mathbb{P}_{k-1}(K)$.

- $\mathcal{C} \mathcal{R}_{k}^{+}, k \geq 2[19]: W_{\mathcal{C R}_{k}^{+}}\left(K, \mathbb{R}^{2}\right)=W_{\mathcal{C R}_{k}}\left(K, \mathbb{R}^{2}\right)+b_{K} \nabla \mathbb{P}_{k}(K)$, where $b_{K}=\lambda_{1} \lambda_{2} \lambda_{3}$ are the bubble functions defined by the barycentric coordinates $\lambda_{i}$ of the triangle $K$, and $Q_{\mathcal{C R}_{k}^{+}}(K)=\mathbb{P}_{k}(K)$. 
TABLE 1. Some known methods for triangular (T) and quadrilateral (Q) reference elements $\hat{K}$ : FE spaces $S(\hat{K}, \mathbb{M}), U\left(\hat{K}, \mathbb{R}^{2}\right)$, and $Q(\hat{K})$ for tensor, displacement and rotation, the associated Poisson-compatible and Stokes-compatible FE pairs used in their construction and stability analyses, and their accuracy parameters $(t, r)$ verify (4.6).

\begin{tabular}{|c|c|c|c|c|c|c|c|}
\hline$\hat{K}$ & Poisson & Stokes & $S(\hat{K}, \mathbb{M})$ & $U\left(\hat{K}, \mathbb{R}^{2}\right)$ & $Q(\hat{K})$ & $(t, r)$ & Ref. \\
\hline \multirow{4}{*}{$\mathrm{T}$} & $\mathcal{B D} \mathcal{M}_{k}$ & - & $\mathbb{P}_{k}^{\partial}(\hat{K}, \mathbb{M}) \oplus \stackrel{\circ}{\mathbb{P}}_{k}(\hat{K}, \mathbb{M})$ & $\mathbb{P}_{k-1}\left(\hat{K}, \mathbb{R}^{2}\right)$ & $\mathbb{P}_{k-1}(\hat{K})$ & $(-1,-1)$ & {$[3]$} \\
\hline & $\mathcal{B D}_{\mathcal{M}}^{+}$ & $\mathcal{C R}_{k+1}$ & $\mathbb{P}_{k}^{\partial}(\hat{K}, \mathbb{M}) \oplus \dot{\mathbb{P}}_{k+1}(\hat{K}, \mathbb{M})$ & $\mathbb{P}_{k}\left(\hat{K}, \mathbb{R}^{2}\right)$ & $\mathbb{P}_{k}(\hat{K})$ & $(0,0)$ & [19] \\
\hline & ${\mathcal{B D} \mathcal{M}_{k}^{++}}^{++}$ & $\mathcal{C R}_{k+1}^{+}$ & $\mathbb{P}_{k}^{\partial}(\hat{K}, \mathbb{M}) \oplus \overrightarrow{\mathbb{P}}_{k+2}(\hat{K}, \mathbb{M})$ & $\mathbb{P}_{k+1}\left(\hat{K}, \mathbb{R}^{2}\right)$ & $\mathbb{P}_{k+1}(\hat{K})$ & $(1,1)$ & [19] \\
\hline & $\mathcal{R} \mathcal{T}_{[k]}$ & $\mathcal{G R}_{[k+1]}$ & $S_{\mathcal{R} \mathcal{T}_{[k]}}(\hat{K}, \mathbb{M})=\mathbb{Q}_{k+1, k}(\hat{K}, \mathbb{M}) \times \mathbb{Q}_{k, k+1}(\hat{K}, \mathbb{M})$ & $\mathbb{Q}_{k, k}\left(\hat{K}, \mathbb{R}^{2}\right)$ & $\mathbb{P}_{k}(\hat{K})$ & $(0,0)$ & {$[5]$} \\
\hline Q & $\overline{\mathcal{R} \mathcal{T}_{[k]}^{+}}$ & $\mathcal{G R}_{[k+1]}^{+}$ & $S_{\mathcal{R} \mathcal{T}_{[k]}}^{\delta}(\hat{K}, \mathbb{M}) \oplus \dot{S}_{\mathcal{R} \mathcal{T}_{[k+1]}}(\hat{K}, \mathbb{M})$ & $\mathbb{Q}_{k+1, k+1}\left(K, \mathbb{R}^{2}\right)$ & $\mathbb{P}_{k+1}(\hat{K})$ & $(1,1)$ & [19] \\
\hline
\end{tabular}

TABLE 2. Two-scale Stokes-compatible FE pairs $\left\{\mathscr{W}_{\gamma}\left(\Omega_{i}\right), \mathscr{Q}_{\gamma_{i n}}\left(\Omega_{i}\right)\right\}$ : local spaces in $\bar{K} \in \mathcal{T}_{h_{s k}}^{\Omega_{i}}$ for triangular $(\mathrm{T})$ and affine quadrilateral $(\mathrm{Q})$ elements.

\begin{tabular}{llll}
\hline \hline $\bar{K}$ & Spaces & $\mathcal{Q}_{\gamma_{i n}}(\bar{K})$ & $W_{\gamma}\left(\bar{K}, \mathbb{R}^{2}\right)$ \\
\hline $\mathrm{T}$ & $\mathcal{C R}_{\gamma}$ & $\mathbb{P}_{k_{i n}-1}\left(\mathcal{T}_{h_{i n}}^{\bar{K}}\right)$ & $W_{\mathcal{C R}_{k_{s k}+1}}\left(\bar{K}, \mathbb{R}^{2}\right)+\stackrel{\circ}{B}_{\mathcal{C R}_{k_{i n}}}\left(\mathcal{T}_{h_{i n}}^{K}, \mathbb{R}^{2}\right)$ \\
$\mathrm{Q}$ & $\mathcal{G R}_{[\gamma]}$ & $\mathbb{P}_{k_{i n}}\left(\mathcal{T}_{h_{i n}}^{\bar{K}}\right)$ & $W_{\mathcal{G R}_{\left[k_{s k}+1\right]}}\left(\bar{K}, \mathbb{R}^{2}\right)+\stackrel{\circ}{B}_{\mathcal{G R}_{\left[k_{i n}\right]}}\left(\mathcal{T}_{h_{i n}}^{\bar{K}}, \mathbb{R}^{2}\right)$ \\
\hline
\end{tabular}

- For quadrilateral elements $K$ (Girault-Raviart FE pair and enriched version):

- $\mathcal{G} \mathcal{R}_{[k]}, k \geq 1[24]: W_{\mathcal{G} \mathcal{R}_{[k]}}\left(K, \mathbb{R}^{2}\right)=\mathbb{F}_{K}\left(\mathbb{Q}_{k, k}\left(\hat{K}, \mathbb{R}^{2}\right)\right.$ and $Q_{\mathcal{G R}_{[k]}}(K)=\mathbb{P}_{k-1}(K)$.

- $\mathcal{G R}_{[k]}^{+}, k \geq 1[19]: W_{\mathcal{G} \mathcal{R}_{[k]}^{+}}\left(K, \mathbb{R}^{2}\right)=W_{\mathcal{G} \mathcal{R}_{[k]}}\left(K, \mathbb{R}^{2}\right)+\stackrel{\circ}{B}_{k+1}\left(K, \mathbb{R}^{2}\right), \mathscr{Q}_{\mathcal{G R}_{[k]}^{+}}(K)=\mathbb{P}_{k}(K) . \stackrel{\circ}{B}_{k+1}\left(K, \mathbb{R}^{2}\right)$ is mapped from $\stackrel{\circ}{B}_{k+1}\left(\hat{K}, \mathbb{R}^{2}\right)=\left\{b_{\hat{K}} \underline{\hat{w}} ; \underline{\hat{w}} \in \mathbb{Q}_{k-1, k-1}\left(\hat{K}, \mathbb{R}^{2}\right)\right\} ; b_{\hat{K}} \in \mathbb{Q}_{2,2}(\hat{K})$ is a bubble function.

\subsection{Examples of stable MHM-WS $\left(\mathcal{E}_{\gamma}\right)$ methods}

Table 1 summarizes some FE spaces $S(\hat{K}, \mathbb{M}), U\left(\hat{K}, \mathbb{R}^{2}\right)$, and $Q(\hat{K})$ that shall be used to form stable $\mathrm{FE}$ spaces $\mathcal{E}_{\gamma}$ for the stress mixed formulation with reduced symmetry. It also shows the corresponding Poissoncompatible and Stokes-compatible FE spaces used in their construction and stability analysis. These methods are known to be stable in single-level settings, as proved in the indicated references. The following accuracy properties are valid for them with $k \geq 1$ and the indicated parameters $t, r \in\{-1,0,1\}$ :

$$
\mathbb{P}_{k}(\hat{K}, \mathbb{M}) \subset S(\hat{K}, \mathbb{M}), \quad \mathbb{P}_{k+t}\left(\hat{K}, \mathbb{R}^{2}\right) \subset U\left(\hat{K}, \mathbb{R}^{2}\right), \quad \mathbb{P}_{k+r}(\hat{K}) \subset Q(\hat{K}) .
$$

Three particular stable scenarios indicated in Table 1, recently analyzed in [19], are for single-level triangular or quadrilateral meshes (i.e. $h_{i n}=h_{s k}=h$ ) and for enriched internal polynomial degrees $k_{i n}=k_{s k}+1$ or $k_{i n}=k_{s k}+2$. We argue that similar methodology may be successfully applied to more general two-scale composite FE space settings $\mathcal{E}_{\gamma}$.

In fact, for the enforcement of the Stokes-constraint (4.2), one may build two-scale composite Stokescompatible FE pairs by adding bubble vector functions to the velocity spaces $W_{\mathcal{C R}_{k_{s k}+1}}\left(\bar{K}, \mathbb{R}^{2}\right)$ of the CrouzeixRaviart spaces for triangles, or of the Girault-Raviart family $W_{\mathcal{G R}_{\left[k_{s k}+1\right]}}\left(\bar{K}, \mathbb{R}^{2}\right)$ for affine quadrilaterals, defined in the coarsest elements $\bar{K} \in \mathcal{T}_{h_{s k}}^{\Omega_{i}}$ (see [9]). These extra terms are defined by the multiplication of appropriate vector spaces, containing the gradient of the enlarged pressure elements, by a fixed scalar bubble function defined for each $\bar{K} \in \mathcal{T}_{h_{s k}}^{\Omega_{i}}$. The next two sections describe the construction of these two-scale Stokes-compatible FE pairs. As far as we understand, they are new in the literature. 


\subsubsection{Stokes-constraint for FE spaces $\mathcal{E}_{\mathcal{B D} \mathcal{M}_{\gamma}}\left(k_{i n} \geq 2\right)$ for triangular meshes}

Since $k_{i n} \geq 2$, the property $\mathscr{U}_{r m} \subset \mathscr{U}_{\gamma_{i n}}$, required by the MHM-WS $\left(\mathcal{E}_{\mathcal{B D} \mathcal{M}_{\gamma}}\right)$ scheme, holds. The particular cases based on the conformal coarse partitions $\mathcal{T}_{h_{s k}}^{\Omega_{i}}\left(h_{i n}=h_{s k}\right)$, and polynomial increment $k_{i n}=k_{s k}+n$, for $n=1,2$, correspond to the FE spaces denoted by $\mathcal{E}_{\mathcal{B D} \mathcal{M}_{\gamma_{s k}}^{+}}$and $\mathcal{E}_{\mathcal{B D} \mathcal{M}_{\gamma_{s k}}^{++}}$considered in [19]. For these methods, the composite rotation space of piecewise polynomials $\mathscr{Q}_{\mathcal{B D} \mathcal{M}_{\gamma_{i n}}}\left(\Omega_{i}\right):=\mathbb{P}_{k_{i n}-1}\left(\mathcal{T}_{h_{s k}}^{\Omega_{i}}\right)$ is stable. This choice is guided by considering the Stokes-compatible Crouzeix-Raviart spaces $\mathcal{C} \mathcal{R}_{\gamma}\left(\Omega_{i}\right)$, with FE spaces

$$
\mathscr{W}_{\mathcal{C} \mathcal{R}_{\gamma}}\left(\Omega_{i}\right) \subset H^{1}\left(\Omega_{i}, \mathbb{R}^{2}\right) \quad \text { and } \quad \mathscr{Q}_{\mathcal{C} \mathcal{R}_{\gamma_{i n}}}\left(\Omega_{i}\right) \subset L^{2}\left(\Omega_{i}\right)
$$

and local FE spaces on each coarse element $\bar{K} \in \mathcal{T}_{h_{s k}}^{\Omega_{i}}$ defined as

$$
\mathscr{W}_{\mathcal{C} \mathcal{R}_{\gamma}}(\bar{K})=W_{\mathcal{C R}_{k_{i n}}}\left(\bar{K}, \mathbb{R}^{2}\right):=\mathbb{P}_{k_{i n}}\left(\bar{K}, \mathbb{R}^{2}\right)+b_{\bar{K}} \mathbb{P}_{k_{s k}-1}\left(\bar{K}, \mathbb{R}^{2}\right) \quad \text { and } \quad \mathscr{Q}_{\mathcal{C R}_{\gamma_{i n}}}(\bar{K}):=\mathbb{P}_{k_{i n}-1}(\bar{K})
$$

for velocity and pressure, respectively, where $b_{\bar{K}}$ is the basic bubble function on $\bar{K}$.

Let us consider now general two-scale scenarios $\gamma=\left(\gamma_{s k}, \gamma_{i n}\right)$, using internal polynomial degree increment $k_{i n}=k_{s k}+n, n \geq 1$ and internal refined partitions $\mathcal{T}_{h_{i n}}^{\Omega_{i}}$, with $h_{i n}=h_{s k} / 2^{\ell}, \ell \geq 0$. The stability of the newly proposed two-scale FE space settings $\mathcal{E}_{\mathcal{B D}} \mathcal{M}_{\gamma}$ also requires two-scale Stokes-compatible Crouzeix-Raviart spaces $\mathscr{W}_{\mathcal{C} \mathcal{R}_{\gamma}}\left(\Omega_{i}\right) \subset H^{1}\left(\Omega_{i}, \mathbb{R}^{2}\right)$ and $\mathscr{Q}_{\mathcal{C R}_{\gamma_{i n}}}\left(\Omega_{i}\right) \subset L^{2}\left(\Omega_{i}\right)$. They are defined on $\bar{K} \in \mathcal{T}_{h_{s k}}^{\Omega_{i}}$ by the composite space $\mathscr{Q}_{\mathcal{C R}_{\gamma_{i n}}}(\bar{K})=\mathbb{P}_{k_{i n}-1}\left(\mathcal{T}_{h_{i n}}^{\bar{K}}\right)$ for pressure, piecewise-defined over the refined partition $\mathcal{T}_{h_{i n}}^{\bar{K}}$ induced on $\bar{K}$, and the velocity space $W_{\mathcal{C R}_{k_{s k}+1}}\left(\bar{K}, \mathbb{R}^{2}\right)+\stackrel{\circ}{B}_{\mathcal{C R}_{k_{i n}}}\left(\mathcal{T}_{h_{i n}}^{\bar{K}}, \mathbb{R}^{2}\right)$, where the stabilizing bubble spaces are

$$
\stackrel{\circ}{B}_{\mathcal{C R}_{k_{i n}}}\left(\mathcal{T}_{h_{i n}}^{\bar{K}}, \mathbb{R}^{2}\right)=\left\{\underline{w} \in H^{1}\left(\bar{K}, \mathbb{R}^{2}\right) ;\left.\underline{w}\right|_{K}=b_{K} \nabla \mathbb{P}_{k_{i n}-1}(K), K \in \mathcal{T}_{h_{i n}}^{\bar{K}}\right\} .
$$

Using these local FE pairs, the requirements of the corollary of Theorem 2 in [9] are fulfilled, and the Stokescompatibility of the resulting two-scale space configuration holds. Furthermore, $\underline{\underline{\nabla}} \times \stackrel{\circ}{B}_{\mathcal{C R}_{k_{i n}}}\left(\mathcal{T}_{h_{i n}}^{\bar{K}}, \mathbb{R}^{2}\right)$ are divergence-free bubble functions in $\bar{K} \in \mathcal{T}_{h_{s k}}^{\Omega_{j}}$, with degree $k_{i n}$ and,

therefore, the required property holds

$$
\underline{\underline{\nabla}} \times \mathscr{W}_{\mathcal{C} \mathcal{R}_{\gamma}}\left(\Omega_{i}\right) \subset \mathscr{S}_{B D M k \gamma}\left(\Omega_{i}\right)=\mathscr{S}_{\mathcal{B D} \mathcal{M}_{\gamma}}^{\partial}\left(\Omega_{i}\right) \oplus \dot{\mathscr{S}}_{\mathcal{B D} \mathcal{M}_{\gamma_{i n}}}\left(\Omega_{i}\right),
$$

which implies that the composite rotation space $\mathscr{Q}_{\mathcal{B D} \mathcal{M}_{\gamma_{i n}}}\left(\Omega_{i}\right):=\mathbb{P}_{k_{i n}-1}\left(\mathcal{T}_{h_{i n}}^{\Omega_{i}}\right)$ is a stable choice for $\mathcal{E}_{\mathcal{B D} \mathcal{M}_{\gamma}}\left(\Omega_{i}\right)$.

\subsubsection{Stokes-constraint for two-scale FE spaces $\mathcal{E}_{\mathcal{R} \mathcal{T}_{[\gamma]}}$ for affine quadrilateral meshes}

Firstly, let us recall the specific FE space setting for $\gamma=\left(\gamma_{s k}, \gamma_{i n}\right)$ based on conformal quadrilateral partitions $\mathcal{T}_{h_{s k}}^{\Omega_{i}}\left(h_{i n}=h_{s k}\right)$ and polynomial increment $k_{i n}=k_{s k}+1$. It corresponds to the case $\mathcal{E}_{\mathcal{R} \mathcal{T}_{\left[\gamma_{s k}\right]}^{+}}$ considered in [19]. For them, we obtain stable rotation spaces $\mathscr{Q}_{\mathcal{R} \mathcal{T}_{\left[\gamma_{i n}\right]}}\left(\Omega_{i}\right)=\mathbb{P}_{\gamma_{i n}}\left(\mathcal{T}_{h_{s k}}^{\Omega_{i}}\right)$ by considering enriched Stokes-compatible Girault-Raviart spaces $\mathcal{G} \mathcal{R}_{\left[k_{s k}+1\right]}^{+}\left(\Omega_{i}\right)$, with local FE spaces $W_{\mathcal{G R}_{\left[k_{s k}+1\right]}^{+}}\left(\bar{K}, \mathbb{R}^{2}\right)=$ $\mathbb{Q}_{k_{s k}+1, k_{s k}+1}\left(\bar{K}, \mathbb{R}^{2}\right)+\stackrel{\circ}{B}_{\mathcal{G R}_{\left[k_{s k}+1\right]}}\left(\bar{K}, \mathbb{R}^{2}\right)$ for velocity, and $\mathbb{P}_{k_{s k}+1}(\bar{K})$ for pressure, in $\bar{K} \in \mathcal{T}_{h_{s k}}^{\Omega_{i}}$. Functions $\underline{w}$ in the bubble spaces ${\stackrel{\circ}{B} \mathcal{G R}_{\left[k_{s k}+1\right]}}\left(\bar{K}, \mathbb{R}^{2}\right)$ are written as $\underline{w}=b_{\bar{K}} \mathbb{Q}_{k_{s k}, k_{s k}}(\bar{K})$, where $b_{\bar{K}}$ is the basic bubble function on $\bar{K}$.

This enrichment methodology can also be extended to prove stability for general local FE spaces $\mathcal{E}_{\mathcal{R}} \mathcal{T}_{[\gamma]}$, for $\gamma=\left(\gamma_{s k}, \gamma_{i n}\right)$, using both non-trivial internal polynomial degree increment $k_{i n}=k_{s k}+n, n \geq 0$, and partition refinement $h_{i n}=h_{s k} / 2^{\ell}, \ell \geq 0$. We obtain stable rotation spaces $\mathscr{Q}_{\mathcal{R} \mathcal{T}_{\left[\gamma_{i n}\right]}}\left(\Omega_{i}\right):=\mathbb{P}_{k_{i n}}\left(\mathcal{T}_{h_{i n}}^{\Omega_{i}}\right)$ using the FE Stokes pair $\left\{\mathscr{W}_{\mathcal{G} \mathcal{R}_{[\gamma]}}\left(\Omega_{i}\right), \mathscr{Q}_{\mathcal{G} \mathcal{R}_{\left[\gamma_{i n}\right]}}\left(\Omega_{i}\right)\right\} \subset H^{1}\left(\Omega_{i}, \mathbb{R}^{2}\right) \times L^{2}\left(\Omega_{i}\right)$, with local pressure space $\tilde{\mathscr{Q}}_{\mathcal{G} \mathcal{R}_{\left[\gamma_{i n}\right]}}(\bar{K})=\mathbb{P}_{k_{i n}}\left(\mathcal{T}_{h_{i n}}^{\bar{K}}\right)$, on all $\bar{K} \in \mathcal{T}_{h_{s k}}^{\Omega_{i}}$, and local velocity space $W_{\mathcal{G R}_{\left[k_{s k}+1\right]}}\left(\bar{K}, \mathbb{R}^{2}\right)+\stackrel{\circ}{B}_{\mathcal{G R}_{\left[k_{i n}\right]}}\left(\mathcal{T}_{h_{i n}}^{\bar{K}}, \mathbb{R}^{2}\right)$, where the bubble spaces 
$\stackrel{\circ}{B}_{\mathcal{G R}_{\left[k_{i n}\right]}}\left(\mathcal{T}_{h_{i n}}^{\bar{K}}, \mathbb{R}^{2}\right) \subset H^{1}\left(\bar{K}, \mathbb{R}^{2}\right)$ are composed by functions $\underline{w}$ such that $\left.\underline{w}\right|_{K}=b_{K} \mathbb{Q}_{k_{i n}-1, k_{i n}-1}(K), K \in \mathcal{T}_{h_{i n}}^{\bar{K}}$. Since $\mathbb{Q}_{k_{i n}-1, k_{i n}-1}(K)$ contains $\mathbb{P}_{k_{i n}-1}\left(K, \mathbb{R}^{2}\right)=\nabla \mathbb{P}_{k_{i n}}(K)$, and accordingly to the corollary of Theorem 2 in [9], the stability of the resulting enriched Stokes-compatible space configuration holds. Furthermore, the tensors in $\underline{\underline{\nabla}} \times \stackrel{\circ}{B}_{\mathcal{G R}_{\left[k_{i n}\right]}}\left(\mathcal{T}_{h_{i n}}^{\bar{K}}, \mathbb{R}^{2}\right)$ are divergence-free bubble functions piecewise defined in $\bar{K} \in \mathcal{T}_{h_{s k}}^{\Omega_{j}}$, with degree $k_{i n}$. The required property $\underline{\underline{\nabla}} \times \mathscr{W}_{\mathcal{R} \mathcal{T}_{[\gamma]}} \subset \mathscr{S}_{\mathcal{R} \mathcal{T}_{[\gamma]}}$ holds, concluding that $\mathscr{Q}_{\mathcal{R} \mathcal{T}_{\left[\gamma_{i n}\right]}}$ is a stable choice for the rotation space in $\mathcal{E}_{\mathcal{R} \mathcal{T}_{[\gamma]} \text {. }}$

We summarize the results of Section 4 in the following theorem.

Theorem 4.1. The MFEM-WS(E $\left.\mathcal{E}_{\gamma}\right)$ formulation (3.16)-(3.18) is well-posed for any of the two-scale FE spaces

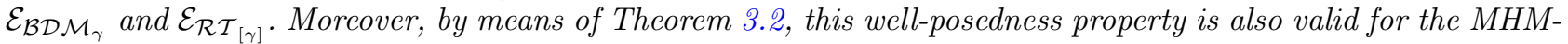
$W S\left(\mathcal{E}_{\gamma}\right)$ scheme defined by (3.6)-(3.15) and based on the respective FE space setting.

\section{Remarks}

- $\mathcal{E}_{\mathcal{B D M}_{\gamma}^{+}}$: for $k_{s k} \geq 1$, these two-scale FE spaces can also be interpreted as two-scale FE spaces $\mathcal{E}_{\mathcal{B D M}_{\gamma}+}$ for $\gamma^{+}=\left(\gamma_{s k}^{+}, \gamma_{i n}^{+}\right)$, with $\gamma_{i n}^{+}=\left(h_{i n}, k_{i n}+1\right)$. Thus, the stability proved to be valid for the later case also holds for the family $\mathcal{E}_{\mathcal{B D} \mathcal{M}_{\gamma}^{+}}$.

$-\mathcal{E}_{\mathcal{R} \mathcal{T}_{[\gamma]}^{+}}$: these two-scale $\mathrm{FE}$ spaces for affine quadrilateral meshes can also be interpreted as two-scale $\mathrm{FE}$ spaces $\mathcal{E}_{\mathcal{R} \mathcal{T}_{\left[\gamma^{+}\right]}}$, for $\gamma^{+}=\left(\gamma_{s k}, \gamma_{i n}^{+}\right)$, with $\gamma_{i n}^{+}=\left(h_{i n}, k_{i n}+1\right)$. Thus, the stability property valid for the latter cases also holds for the family $\mathcal{E}_{\mathcal{R} \mathcal{T}_{[\gamma]}^{+}}$.

\section{UNIFIED ERROR ANALYSIS FOR THE MHM-WS $\left(\mathcal{E}_{\gamma}\right)$ METHOD}

In this section, we present a unified error analysis for stable MHM-WS( $\left.\mathcal{E}_{\gamma}\right)$ methods for two-scale FE spaces $\mathcal{E}_{\gamma}$ via the equivalent MFEM-WS $\left(\mathcal{E}_{\gamma}\right)$ formulations, by means of Theorem 3.2. The analysis is general enough to be applied to the stable families $\mathcal{E}_{\mathcal{B D} \mathcal{M}_{\gamma}}$ and $\mathcal{E}_{\mathcal{R}} \mathcal{T}_{[\gamma]}$, but also to other stable two-scale FE spaces eventually constructed under similar circumstances, in association to other kinds of Poisson-compatible FE pairs.

A well-known methodology for error analysis of MFEM-WS $\left(\mathcal{E}_{\gamma}\right)$ formulations requires the construction of appropriate interpolants, as proposed in [6]. The error estimates are then bounded in terms of the interpolation errors. The particular two-scale interpolants of interest are discussed in the next theorem.

Theorem 5.1. Let $\mathcal{E}_{\gamma}=\mathscr{S}_{\gamma} \times \mathscr{U}_{\gamma_{i n}} \times \mathscr{Q}_{\gamma_{i n}}$ be FE spaces verifying the stability constraints (4.1) and (4.2).

(1) There exists an interpolant $\Pi \frac{\sigma}{\bar{\gamma}}: H^{1}(\Omega, \mathbb{M}) \rightarrow \mathscr{S}_{\gamma}$, satisfying

$$
\begin{aligned}
& (\underline{\nabla} \cdot(\underline{\underline{\tau}}-\Pi \underline{\underline{\bar{\gamma}}} \underline{\underline{\tau}}), \underline{v})+(\operatorname{asym}(\underline{\underline{\tau}}-\Pi \underline{\underline{\underline{\gamma}}} \underline{\underline{\tau}}), \varphi)=0, \forall \underline{v} \in \mathscr{U}_{\gamma_{i n}}, \forall \varphi \in \mathscr{Q}_{\gamma_{i n}}, \\
& \|\Pi \underline{\bar{\gamma}} \underline{\underline{\underline{\tau}}}\|_{H(\operatorname{div}, \Omega, \mathbb{M})} \lesssim\|\underline{\underline{\underline{\tau}}}\|_{H(\operatorname{div}, \Omega, \mathbb{M})} .
\end{aligned}
$$

(2) For a sufficiently smooth tensor $\underline{\underline{\tau}}$, the interpolation error estimate reads

$$
\|\underline{\underline{\tau}}-\Pi \underline{\underline{\underline{\sigma}}} \underline{\underline{\underline{\tau}}}\|_{\mathbf{L}^{2}(\Omega, \mathbb{M})} \lesssim h_{s k}^{k_{s k}+1}\|\underline{\underline{\tau}}\|_{H^{k_{s k}+1}(\Omega, \mathbb{M})} .
$$

The leading constants appearing on the right sides of estimates (5.2) and (5.3) are independent of $\gamma$.

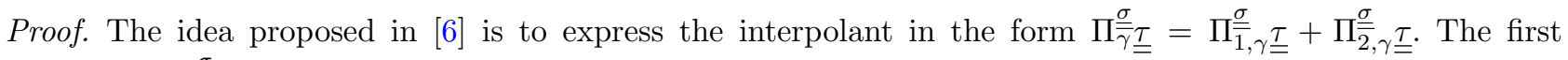
component $\Pi_{1, \gamma}^{\underline{\underline{\sigma}}} \underline{\underline{\tau}}$ verifies the divergence commutative property expressed by $(5.1)$ when taking $\varphi=0$. It is row-wisely defined in the spirit of standard projection-based interpolants $\boldsymbol{\pi}_{\gamma}^{D}: H^{s}\left(\Omega, \mathbb{R}^{2}\right) \rightarrow \mathscr{V}_{\gamma}$ adopted for two-scale Poisson-compatible pairs $\left\{\mathscr{V}_{\gamma}, \mathscr{P}_{\gamma_{i n}}\right\}$, with enhanced bubble flux components (see $\left.[19,20]\right)$. Recalling 
the definition of $\boldsymbol{\pi}_{\gamma}^{D}$ for the single-level case (4.3)-(4.5), the two-scale version becomes $\boldsymbol{\pi}_{\gamma}^{D} \underline{\eta}=\boldsymbol{\pi}_{\gamma}^{D, \partial} \underline{\eta}+\dot{\boldsymbol{\pi}}_{\gamma_{i n}}^{D}(\underline{\eta}-$ $\left.\boldsymbol{\pi}_{\gamma}^{D, \partial} \underline{\eta}\right)$, where only the internal interpolant $\stackrel{\circ}{\boldsymbol{\pi}}_{\gamma_{i n}}^{D}$ has to be updated. A uniform bound for $\left\|\Pi_{1, \gamma}^{\underline{\underline{\sigma}}} \underline{\underline{\tau}}\right\|_{H(\operatorname{div}, \Omega, \mathbb{M})}$, independent of $\gamma$, follows from the same property valid for $\left\|\boldsymbol{\pi}_{\gamma}^{D}\right\|_{H\left(\operatorname{div}, \Omega, \mathbb{R}^{2}\right)}$.

The second interpolant $\Pi_{2}^{\underline{\underline{\sigma}}}, \underline{\underline{\tau}}$ should verify the commutative property (5.2) when $\underline{v}=0$. It is defined following similar arguments applied in [6] for the single-level cases. Namely, consider the Stokes-compatible pair $\left\{\mathscr{W}_{\gamma}, \mathscr{Q}_{\gamma_{i n}}\right\}$. This pair exists since the stability Stokes-constraint is satisfied. Let $\phi=\left[\begin{array}{ll}\phi_{1} & \phi_{2}\end{array}\right] \in \mathscr{W}_{\gamma}$ be the solution of the Stokes problem with divergence constraint $-(\nabla \cdot \underline{\phi}, \varphi)=\left(\operatorname{asym}\left(\Pi_{1}^{\underline{\underline{\sigma}}}, \underline{\underline{\tau}}-\underline{\underline{\tau}}\right), \varphi\right), \forall \varphi \in \mathscr{Q}_{\gamma_{i n}}$, and define $\Pi_{2, \gamma}^{\underline{\underline{\sigma}}} \underline{\underline{\tau}}=\underline{\underline{\nabla}} \times \underline{\phi}=\left[\begin{array}{c}\partial_{2} \phi_{1}-\partial_{1} \phi_{1} \\ \partial_{2} \phi_{2}-\partial_{1} \phi_{2}\end{array}\right] \in \mathscr{S}_{\gamma}$. Therefore, $\Pi \underline{\overline{\underline{\sigma}}, \gamma} \underline{\underline{\underline{\tau}}}$ is divergence free and

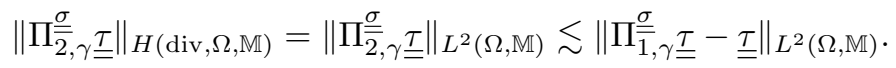

Since asym $\Pi_{2}^{\underline{\underline{\sigma}}}, \underline{\underline{\tau}}=-\partial_{1} \phi_{1}-\partial_{2} \phi_{2}=-\nabla \cdot \underline{\phi}$, so that the required commutative property holds.

Concerning the error estimate (5.2), we first observe that it holds for $\Pi_{1, \gamma}^{\underline{\sigma}}$. In fact, this is a consequence of similar error estimate valid for $\boldsymbol{\pi}_{\gamma}^{D}$ of the associated two-scale FE Poisson-compatible space, proved in [20], the leading constant appearing on the right side only depending on the shape-regularity factors of the meshes $\mathcal{T}_{h_{i n}}^{\Omega_{i}}$, which are supposed to be independent of the mesh-widths, and on the bound for the projection $\hat{\boldsymbol{\pi}}^{D}$ on the corresponding reference element $\hat{K}$ (see also Thm. 4.1 in [2]).

We conclude the proof after using the triangular inequality and the estimate (5.4).

Error estimates for the $\operatorname{MHM}-\mathrm{WS}\left(\mathcal{E}_{\gamma}\right)$ method use both discretization parameters $\gamma_{s k}$ and $\gamma_{i n}$ set in Section 3.1. Moreover, the elliptic regularity property, which is known to hold in a variety of circumstances of planar elasticity on convex domains $\Omega$ [13], is used for the error estimate in $\underline{u}$. Namely, if $\underline{\underline{v}}=\underline{\underline{A}}^{-1} \underline{\underline{\varepsilon}}(\underline{w}) \in H(\operatorname{div}, \Omega, \mathbb{S})$ is the solution of the elasticity problem $\underline{\nabla} \cdot \underline{\underline{v}}=\underline{\theta}$, with boundary condition $\underline{w}=\underline{0}$ on $\partial \bar{\Omega}$, we assume that

$$
\|\underline{\underline{v}}\|_{H^{1}(\Omega, \mathbb{M})}+\|\underline{w}\|_{H^{2}\left(\Omega, \mathbb{R}^{2}\right)} \lesssim\|\underline{\theta}\|_{L^{2}\left(\Omega, \mathbb{R}^{2}\right)} .
$$

Theorem 5.2. Suppose $\mathcal{E}_{\gamma}=\mathscr{S}_{\gamma} \times \mathscr{U}_{\gamma_{i n}} \times \mathscr{Q}_{\gamma_{i n}}$ is a two-scale FE space setting verifying the stability constraints (4.1) and (4.2) and the accuracy properties (4.6), and let $(\underline{\underline{\tilde{\sigma}}}, \underline{\underline{\tilde{u}}}, \tilde{q}) \in \mathscr{S}_{\gamma} \times \mathscr{U}_{\gamma_{i n}} \times \in \mathscr{Q}_{\gamma_{i n}}$ be the approximate solution recovered from the output of the $M H M-W S\left(\mathcal{E}_{\gamma}\right)$ method, as in (3.5). Assume the regularity property (5.5) holds.

(1) Then, the next estimates are valid:

$$
\begin{aligned}
&\|\underline{\underline{\sigma}}-\underline{\underline{\sigma}}\|_{\mathbf{L}^{2}(\Omega, \mathbb{M})}+\|q-\tilde{q}\|_{L^{2}(\Omega)} \lesssim h_{s k}^{k_{s k}+1}\|\underline{\underline{\sigma}}\|_{H^{k_{s k}+1}(\Omega, \mathbb{M})}+h_{i n}^{k_{i n}+r+1}\|q\|_{H^{k_{i n}+r+1}(\Omega)}, \\
&\|\underline{\nabla} \cdot(\underline{\underline{\sigma}}-\underline{\underline{\sigma}})\|_{L^{2}\left(\Omega, \mathbb{R}^{2}\right)} \lesssim h_{i n}^{k_{i n}+t+1}\|\underline{\nabla} \cdot \underline{\underline{\sigma}}\|_{H^{k_{i n}+t+1}\left(\Omega, \mathbb{R}^{2}\right)}, \\
&\|\underline{u}-\underline{\tilde{u}}\|_{L^{2}\left(\Omega, \mathbb{R}^{2}\right)} \lesssim h_{s k}^{k_{s k}+2}\|\underline{\underline{\sigma}}\|_{H^{k_{i n}+t+1}(\Omega, \mathbb{M})}+h_{i n}^{k_{i n}+t+1}\|\underline{u}\|_{H^{k_{i n}+t+1}\left(\Omega, \mathbb{R}^{2}\right)} \\
&+h_{s k} h_{i n}^{k_{i n}+r+1}\|q\|_{H^{k_{i n}+r+1}(\Omega)}
\end{aligned}
$$

where the exact fields $\underline{\sigma}, \underline{u}$ and $q$ are regular enough for the norms to make sense.

(2) The above estimates hold for the $M H M-W S\left(\mathcal{E}_{\gamma}\right)$ formulations using $\mathcal{E}_{\mathcal{R} \mathcal{T}_{[\gamma]}}$ and $\mathcal{E}_{\mathcal{B D} \mathcal{M}_{\gamma}^{+}} F E$ spaces, with $t=r=0$, and using $\mathcal{E}_{\mathcal{B D M}_{\gamma}} F E$ spaces for $k_{i n}>1$, with $t=r=-1$.

Proof. By means of Theorem 3.2, we derive the error estimates for the equivalent $\operatorname{MFEM-WS}\left(\mathcal{E}_{\gamma}\right)$ formulation, for which the following estimates in terms of interpolation errors hold (see [19] or the references therein):

$$
\begin{aligned}
\|\underline{\underline{\sigma}}-\underline{\underline{\sigma}}\|_{L^{2}(\Omega, \mathbb{M})}+\|q-\tilde{q}\|_{L^{2}(\Omega)} & \lesssim\|\underline{\underline{\sigma}}-\Pi \underline{\underline{\bar{\gamma}}} \underline{\underline{\sigma}}\|_{L^{2}(\Omega, \mathbb{M})}+\left\|q-\Pi_{\gamma_{i n}}^{q} q\right\|_{L^{2}(\Omega)}, \\
\|\underline{\nabla} \cdot(\underline{\underline{\sigma}}-\underline{\underline{\sigma}})\|_{L^{2}\left(\Omega, \mathbb{R}^{2}\right)} & \lesssim\|\underline{\underline{\nabla}} \cdot(\underline{\underline{\sigma}}-\Pi \underline{\bar{\gamma}} \underline{\underline{\sigma}})\|_{L^{2}\left(\Omega, \mathbb{R}^{2}\right)}, \\
\left\|\Pi \overline{\underline{\gamma}}_{i n} \underline{u}-\underline{\underline{u}}\right\|_{L^{2}\left(\Omega, \mathbb{R}^{2}\right)}^{2} & =(\underline{\underline{A}}(\underline{\underline{\sigma}}-\underline{\underline{\tilde{\sigma}}}), \underline{\underline{v}}-\Pi \underline{\bar{\gamma}} \underline{\underline{v}})+\left(\Pi_{\gamma_{i n}}^{q} q-q, \operatorname{asym}\left(\underline{\underline{v}}-\Pi \frac{\overline{\underline{\gamma}}}{\underline{\underline{v}}}\right)\right),
\end{aligned}
$$


where $\Pi \frac{\bar{\sigma}}{\bar{\gamma}}: H^{1}(\Omega, \mathbb{M}) \rightarrow \mathscr{S}_{\gamma}$ is the interpolant defined in Section $5.1, \Pi_{\gamma_{i n}}^{\underline{u}}: L^{2}\left(\Omega, \mathbb{R}^{2}\right) \rightarrow \mathscr{U}_{\gamma_{i n}}$ and $\Pi_{\gamma_{i n}}^{q}:$ $L^{2}\left(\Omega, \mathbb{R}^{2}\right) \rightarrow \mathscr{Q}_{\gamma_{i n}}$ are $L^{2}$-orthogonal-projections, and $\underline{\underline{v}}=\underline{\underline{A}}^{-1} \underline{\underline{\varepsilon}}(\underline{w}) \in H(\operatorname{div}, \Omega, \mathbb{S})$ is the solution of the elasticity problem $\underline{\nabla} \cdot \underline{\underline{A}}^{-1} \underline{\underline{\varepsilon}}(\underline{w})=\Pi \frac{u}{\gamma_{i n}} \underline{u}-\underline{\tilde{u}}$ with homogeneous boundary condition $\underline{w}=\underline{0}$ on $\partial \Omega$. Being a $L^{2}$-projections, $\Pi_{\gamma_{i n}}^{u}$ and $\Pi_{\gamma_{\text {in }}}^{q}$ have unitary norm, and the following error estimates hold

$$
\begin{aligned}
&\left\|\underline{v}-\Pi \frac{u}{\gamma_{i n}} \underline{v}\right\|_{L^{2}\left(\Omega, \mathbb{R}^{2}\right)} \lesssim h_{i n}^{k_{i n}+t+1}\|\underline{v}\|_{H^{k_{i n}+t+1}\left(\Omega, \mathbb{R}^{2}\right)}, \\
&\left\|\varphi-\Pi_{\gamma_{i n}}^{q} \varphi\right\|_{L^{2}(\Omega)} \lesssim h_{i n}^{k_{i n}+r+1}\|\varphi\|_{H^{k_{i n}+r+1}(\Omega)} .
\end{aligned}
$$

Due to the divergence-consistency property, meaning that $\underline{\nabla} \cdot \Pi \stackrel{\overline{\bar{\gamma}}}{\underline{\underline{\tau}}}$ is the $L^{2}$-projection of $\underline{\nabla} \cdot \underline{\underline{\tau}}$ over $\mathscr{U}_{\gamma_{i n}}$, then

$$
\|\underline{\nabla} \cdot(\underline{\underline{\tau}}-\Pi \underline{\underline{\underline{\gamma}}} \underline{\underline{\tau}})\|_{L^{2}\left(\Omega, \mathbb{R}^{2}\right)} \lesssim h_{i n}^{k_{i n}+t+1}\|\underline{\nabla} \cdot \underline{\underline{\tau}}\|_{H^{k_{i n}+t+1}\left(\Omega, \mathbb{R}^{2}\right)} \cdot
$$

Consequently, estimates (5.6) and (5.7) follow directly by inserting the interpolation errors (5.3), (5.13), and (5.14) in (5.9) and (5.10). Using Cauchy-Schwartz inequality in (5.11), we obtain

$$
\left\|\Pi \frac{u}{\gamma_{i n}} \underline{u}-\underline{\underline{u}}\right\|_{L^{2}\left(\Omega, \mathbb{R}^{2}\right)}^{2} \leq\|\underline{\underline{A}}(\underline{\underline{\sigma}}-\underline{\underline{\tilde{\sigma}}})\|_{L^{2}(\Omega, \mathbb{M})}\|\underline{\underline{\underline{v}}}-\Pi \underline{\underline{\underline{\gamma}}} \underline{\underline{\underline{v}}}\|_{L^{2}(\Omega, \mathbb{M})}+\left\|\Pi_{\gamma_{i n}}^{q} q-q\right\|_{L^{2}(\Omega)}\|\operatorname{asym}(\underline{\underline{\underline{v}}}-\Pi \underline{\underline{\underline{\gamma}}} \underline{\underline{\underline{v}}})\|_{L^{2}(\Omega)} .
$$

Therefore, we use $\|\underline{\underline{v}}-\Pi \underline{\underline{\underline{\gamma}}} \underline{\underline{\underline{v}}}\|_{L^{2}(\Omega, \mathbb{M})} \lesssim h_{s k}\|\underline{\underline{v}}\|_{H^{1}(\Omega, \mathbb{M})}, \|$ asym $\left(\underline{\underline{v}}-\Pi \frac{\underline{\underline{\gamma}}}{\underline{\underline{v}}}\right)\left\|_{L^{2}(\Omega)} \lesssim h_{s k}\right\| \underline{\underline{v}} \|_{H^{1}(\Omega, \mathbb{M})}$, and the estimate $\|\underline{\underline{v}}\|_{H^{1}(\Omega, \mathbb{M})} \lesssim\left\|\Pi \frac{u}{\gamma_{i n}} \underline{u}-\underline{\tilde{u}}\right\|_{L^{2}\left(\Omega, \mathbb{R}^{2}\right)}$, given by elliptic regularity property (5.5), to obtain

$$
\left\|\Pi \frac{u}{\gamma_{i n}} \underline{u}-\underline{\tilde{u}}\right\|_{L^{2}\left(\Omega, \mathbb{R}^{2}\right)} \lesssim h_{s k}\left(\|\underline{\underline{\sigma}}-\underline{\underline{\tilde{\sigma}}}\|_{L^{2}(\Omega, \mathbb{M})}+\left\|\Pi_{\gamma_{i n}}^{q} q-q\right\|_{L^{2}(\Omega)}\right) .
$$

Then, we insert the estimate above in the triangle inequality to obtain $\|\underline{u}-\underline{\tilde{u}}\|_{L^{2}\left(\Omega, \mathbb{R}^{2}\right)} \leq\|\underline{u}-\Pi \underline{\underline{u}} \underline{\underline{u}} \underline{u}\|_{L^{2}\left(\Omega, \mathbb{R}^{2}\right)}+$ $\left\|\Pi \underline{\underline{u}} \underline{\gamma}_{i n} \underline{u}-\underline{\tilde{u}}\right\|_{L^{2}\left(\Omega, \mathbb{R}^{2}\right)}$. The estimate (5.8) follows from this last inequality and using the interpolation errors (5.12) and (5.13), and the estimate (5.6). Finally, the second statement follows from the first one, for all hypotheses are satisfied.

\section{Remarks}

(i) The stress error is limited to the order $O\left(h_{s k}^{k_{s k}+1}\right)$, independently of internal enrichment, because the edge terms live in the coarsest scale level $\gamma_{s k}=\left(h_{s k}, k_{s k}\right)$ of the normal traces over the skeleton interfaces.

(ii) Divergence of the stress can reach arbitrary high accuracy orders, profiting from finer meshes and higher polynomial degrees used for the approximations in $\mathscr{U}_{\gamma_{i n}}$.

(iii) Despite the fact that finer meshes and higher polynomial degrees are also used for the approximations in $\mathscr{Q}_{\gamma_{i n}}$, the accuracy of the rotation is limited by the stress accuracy order $O\left(h_{s k}^{k_{s k}+1}\right)$.

(iv) The constants in the above error estimates are independent of the Poisson ratio, a fact allowing to work with materials near the incompressible limit, avoiding the locking phenomena, which is one of main advantages of using stress mixed methods to solve linear elasticity.

(v) Since $\|\underline{\underline{\tau}} \cdot \underline{n}\|_{H^{-\frac{1}{2}}(\Gamma)} \leq\|\underline{\underline{\tau}}\|_{H(\operatorname{div}, \Omega, \mathbb{M})}$, for $\underline{\underline{\tau}} \in \mathscr{S}$, convergence rate for $\|\lambda-\tilde{\lambda}\|_{H^{-\frac{1}{2}(\Gamma)}}$ can be obtained directly from the estimations (5.6), and (5.7) as

$$
\|\lambda-\tilde{\lambda}\|_{H^{-\frac{1}{2}}(\Gamma)} \lesssim h_{s k}^{k_{s k}+1}\|\underline{\underline{\sigma}}\|_{H^{k_{s k}+1}(\Omega, \mathbb{M})}+h_{i n}^{k_{i n}+t+1}\|\underline{\nabla} \cdot \underline{\underline{\sigma}}\|_{H^{k_{i n}+t+1}\left(\Omega, \mathbb{R}^{2}\right)}+h_{i n}^{k_{i n}+r+1}\|q\|_{H^{k_{i n}+r+1}(\Omega)} .
$$

(vi) Due to the $L^{2}$-orthogonality of $\mathscr{U}_{r m}$ and $\mathscr{U}^{\perp}$, the convergence rate (5.8) valid for $\|\underline{u}-\underline{\tilde{u}}\|_{L^{2}\left(\Omega, \mathbb{R}^{2}\right)}$ also holds for $\left\|\underline{u}_{r m}-\underline{\tilde{u}}_{0}\right\|_{L^{2}\left(\Omega, \mathbb{R}^{2}\right)}^{2}$ and $\left\|\underline{u}^{\perp}-\underline{\tilde{u}}^{\perp}\right\|_{L^{2}\left(\Omega, \mathbb{R}^{2}\right)}^{2}$, for

$$
\|\underline{u}-\underline{\tilde{u}}\|_{L^{2}\left(\Omega, \mathbb{R}^{2}\right)}^{2}=\left\|\left(\underline{u}_{r m}-\underline{\tilde{u}}_{r m}\right)+\left(\underline{u}^{\perp}-\underline{\tilde{u}}^{\perp}\right)\right\|_{L^{2}\left(\Omega, \mathbb{R}^{2}\right)}^{2}=\left\|\underline{u}_{r m}-\underline{\tilde{u}}_{r m}\right\|_{L^{2}\left(\Omega, \mathbb{R}^{2}\right)}^{2}+\left\|\underline{u}^{\perp}-\underline{\tilde{u}}^{\perp}\right\|_{L^{2}\left(\Omega, \mathbb{R}^{2}\right)}^{2} .
$$



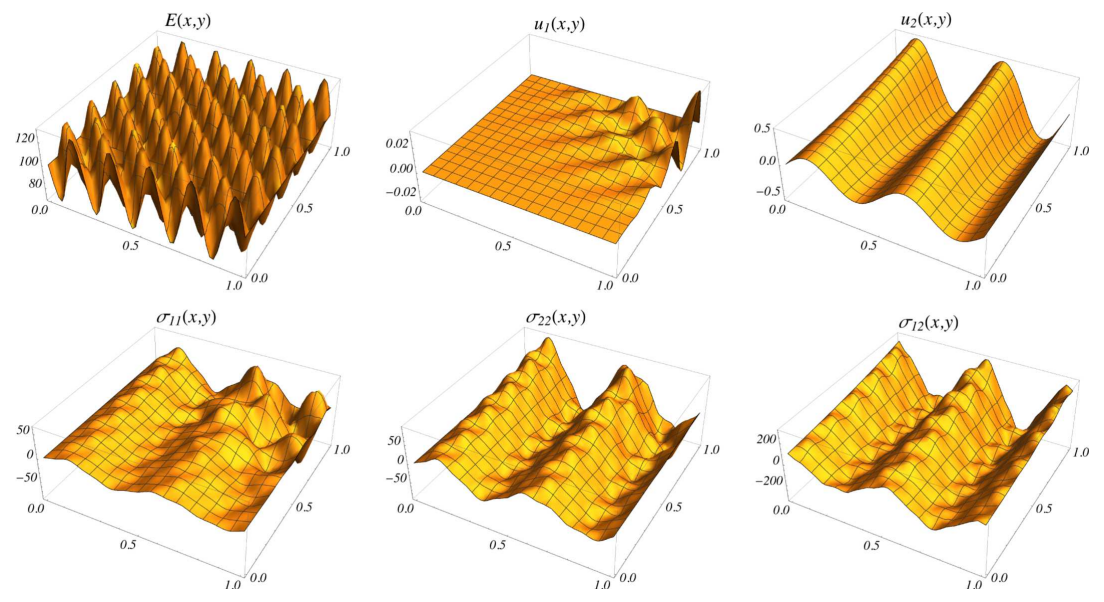

Figure 2. Oscillatory Young's modulus $E$, the components of the analytic displacement $\underline{u}$ and stress tensor $\underline{\underline{\sigma}}$.

\section{NumericAl VERIFICATION TESTS}

In this section, we present and discuss some verification tests for the $\operatorname{MHM}-\mathrm{WS}\left(\mathcal{E}_{\gamma}\right)$ formulation analyzed in the previous sections. The results are compared with the ones given by the coarse single-level MFEM$\mathrm{WS}\left(\mathcal{E}_{\gamma_{s k}}\right)$ formulation, and by the MHM- $H^{1}$ formulation [28,35], that use $H^{1}$-conforming $\mathrm{FE}$ displacement spaces of corresponding two-scale resolution, using the hierarchical shape functions described in [17]. We refer to $[18,39]$ for the implementation of $H(\mathrm{div})$-conforming shape functions of edge and internal types, required in the construction of tensor FE spaces.

For the current simulations, we implemented the methods in the computational framework NeoPZ ${ }^{6}$, where tools for the construction of the required constrained $H$ (div)-conforming spaces are available (e.g., a hierarchy of shape functions of high degree for a variety of element geometry, data structure allowing the identification of face and internal shape functions of different degrees, and procedures for shape function restraints, as the ones usually adopted in adaptive hp-strategies). The upscaling-downscaling stages are crucial for the construction of efficient computational algorithms, mainly because they decompose the resolution of the problem in terms of local expensive (but independent local solvers) and cheaper coupled global systems. We refer to [20,35] for a discussion of different ways to implement MHM methods.

\subsection{Problem 1: An oscillatory Young's modulus case}

Let $\Omega=(0,1) \times(0,1)$ be a isotropic elastic body with Lamé parameters $\lambda=\frac{E(x, y) \nu}{(1+\nu)(1-2 \nu)}$ and $\mu=\frac{E(x, y)}{2(1+\nu)}$ expressed in terms of the oscillatory Young's modulus $E(x, y)=100(1+0.3 \sin (10 \pi(x-0.5)) \cos (10 \pi y)$ and Poisson ratio $\nu=0.3$. The exact displacement $\underline{u}$, vanishing on $\partial \Omega$, and is given by the expression

$$
\underline{u}(x, y)=\left(\begin{array}{c}
\frac{1}{3}\left(\frac{x}{3}\right)^{2} \\
y^{2} \cos (6 \pi x) \sin (7 \pi y) \\
\frac{1}{5} e^{y} \sin (4 \pi x)
\end{array}\right),
$$

from which the body force $\underline{f}$ is derived. Plots for $E$, components $\underline{u}_{i}$, and $\underline{\underline{\sigma}}_{i, j}$ are shown in Figure 2 .

\footnotetext{
${ }^{6} \mathrm{NeoPZ}$ open-source platform: http://github.com/labmec/neopz.
} 

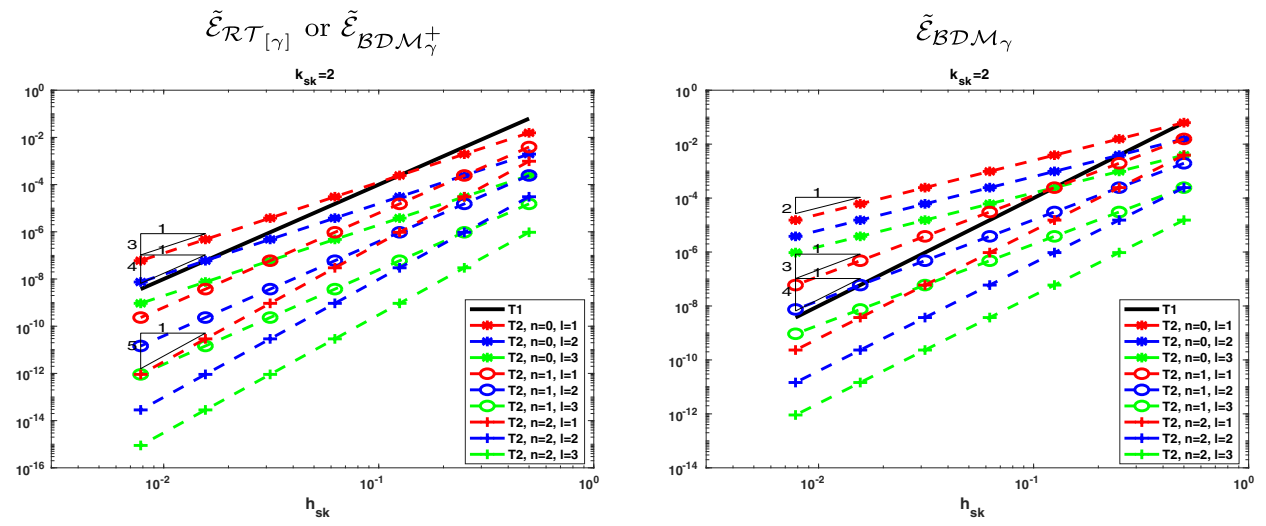

FiguRE 3. Effects of $h_{s k}^{k_{s k}+2}$ in Term 1 (T1), and $h_{i n}^{t+1}$ in Term 2 (T2) in the displacement error estimate: $k_{s k}=2, k_{i n}=k_{s k}+n, n=0,1$, and 2 , for $h_{i n}=h_{s k} / 2^{\ell}, \ell=1,2$, and 3 .

The results shown in this section are for two-scale FE spaces $\mathcal{E}_{\mathcal{R}} \mathcal{T}_{[\gamma]}$, for square meshes, $\mathcal{E}_{\mathcal{B D} \mathcal{M}_{\gamma}}$, and $\mathcal{E}_{\mathcal{B D}^{\prime} \mathcal{M}_{\gamma}^{+}}$, for triangular elements. Two types of curves are shown: mesh-based and space-based convergence histories.

In the mesh-based scenario, convergence rate is based on the usual $H$-refinement of the macro-partition, and use $h_{s k}=H$ (no mesh-skeleton refinement). The goal is to verify the error estimates predicted in Theorem 5.2. Concerning displacement errors, the three terms in the right hand side of (5.8) may have different influence on the results. For instance, for the two-scale families of FE spaces $\mathcal{E}_{\mathcal{R}} \mathcal{T}_{[\gamma]}$ and $\mathcal{E}_{\mathcal{B D} \mathcal{M}_{\gamma}^{+}}$the last term $h_{s k} h_{i n}^{k_{i n}+1}$ is always dominated by $h_{s k}^{k_{s k}+2}$ (Term 1) and $h_{i n}^{k_{s k}+n+1}$ (Term 2) appearing in the first and second terms. Different regimes may be observed for Term 1 and Term 2, depending on parameter configurations. For instance, when internal polynomial degree increment $k_{i n}=k_{s k}+n$ is applied, with $n \geq 1$, the influence of Term 1 dominates Term 2 in the range for $h_{s k}$ illustrated in Figure 3 (left-plots), independently of the internal mesh refinement $h_{i n}=h_{s k} / 2^{\ell}, \ell \geq 1$. For the two-scale FE spaces $\mathcal{E}_{\mathcal{B D} \mathcal{M}_{\gamma}}$, Term 1 dominates Term 2 when $n \geq 2$, as shown in Figure 3 (right-plots). These effects shall be verified in the simulations presented in this section.

Note also that the macro mesh-size $H$ does not appear explicitly in the error estimates of Theorem 5.2. This means that convergence is achieved by making $h_{s k} \rightarrow 0$, even if $\mathcal{T}_{H}$ stays unchanged. This second type of convergence history is called space-based convergence, based on the refinement of the skeleton partitions (as well as the internal ones) while keeping fixed the macro-partition. The purpose is to verify if an extra convergence rate of order $h_{s k}^{1 / 2}$ occurs, as observed in the numerical tests of $[27,35]$ using the MHM- $H^{1}$ method.

\subsubsection{Mesh-based convergence with square elements}

In this part, all verification tests for the oscillatory Young's modulus case are for FE spaces $\mathcal{E}_{\mathcal{R} \mathcal{T}_{[\gamma]}}$ based on square local partitions.

The results for the stress component $\sigma_{11}$ obtained by the application of the $\operatorname{MHM}-\mathrm{WS}\left(\mathcal{E}_{\left.\mathcal{R} \mathcal{T}_{[\gamma]}\right)}\right)$ scheme are displayed in Figure 4, for different configurations of $\gamma$. Precisely, we show plots for: (a) $8 \times 8$ subregions, $H=2^{-3}$, $h_{s k}=H, k_{s k}=2$; (b) $8 \times 8$ subregions, $h_{s k}=H / 4, k_{s k}=0$; and (c) $32 \times 32$ subregions, $H=2^{-5}, h_{s k}=H$, $k_{s k}=2$. In all these cases, $h_{i n}=2^{-7}$, and $k_{i n}=k_{s k}+1$. It is clear that the FE space of the case (a) is not sufficiently refined to capture the essential features of the solution. The other two FE spaces, which are equivalent in terms of element sizes on the edges, show similar approximations, but the errors for the FE space of case (c) are the smallest ones.

Figures 5 and 6 show $L^{2}$-error curves for $\underline{u}, \underline{\underline{\sigma}}, \underline{\nabla} \cdot \underline{\underline{\sigma}}, q$, and energy norm $(\underline{\underline{A}} \underline{\underline{\varepsilon}}(\underline{u}), \underline{\underline{\varepsilon}}(\underline{u}))^{\frac{1}{2}}$, in terms of the macro mesh size $H=2^{-j}, j=1,2, \cdots, 6$, for the MHM-WS( $\left.\mathcal{E}_{\mathcal{R} \mathcal{T}_{[\gamma]}}\right)$ scheme, using $h_{s k}=H, k_{s k}=1$ or 2 , and 

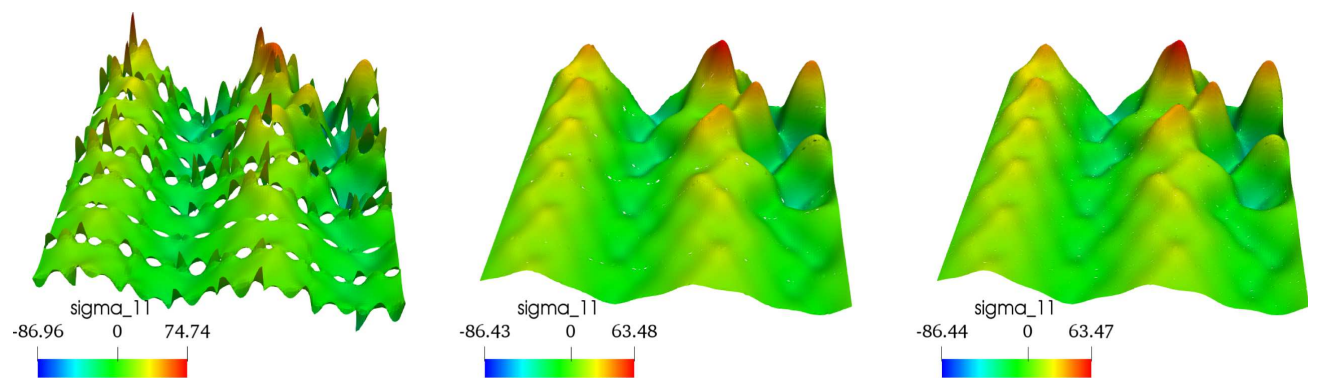

Figure 4. Problem 1 - Tensor component $\sigma_{11}$ solved by the MHM-WS $\left(\mathcal{E}_{\left.\mathcal{R} \mathcal{T}_{[\gamma]}\right)}\right)$ scheme for different square partitions: $8 \times 8$ subregions, $h_{s k}=H, k_{s k}=2$ (left-image); $8 \times 8$ subregions, $h_{s k}=H / 4, k_{s k}=0$ (middle-image); and $32 \times 32$ subregions, $h_{s k}=H$, and $k_{s k}=2$ (right-image). In all the cases $k_{i n}=k_{s k}+1$, and $h_{i n}=2^{-7}$.
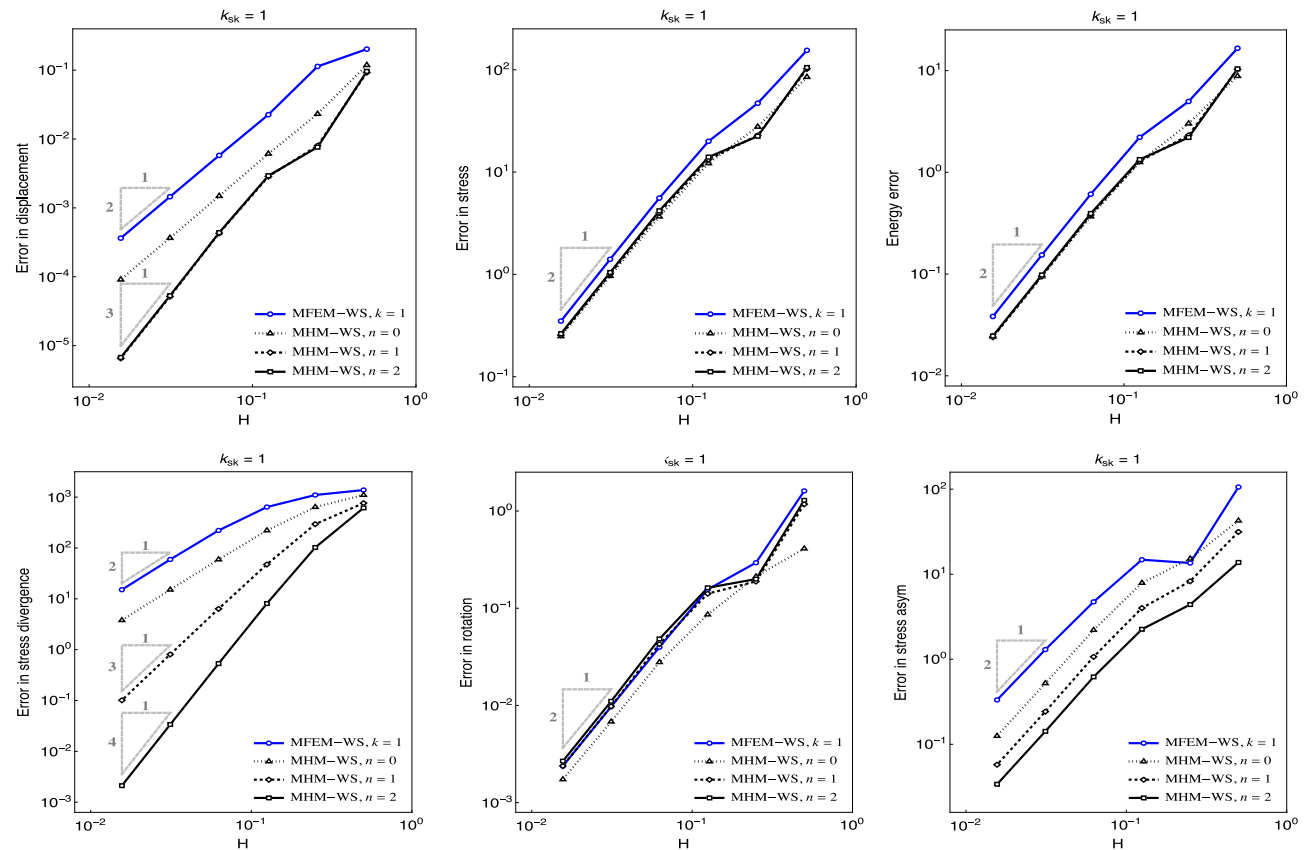

FiguRE 5. Problem 1 - Effect of increasing $k_{i n}$ : MHM-WS $\left(\mathcal{E}_{\mathcal{R}} \mathcal{T}_{[\gamma]}\right)$ scheme with $h_{s k}=H=2^{-j}$, $j=1,2, \cdots 6, h_{i n}=h_{s k} / 2, k_{s k}=1$, and $k_{i n}=k_{s k}+n, n=0,1,2$; single-level MFEM$\mathrm{WS}\left(\mathcal{E}_{\mathcal{R} \mathcal{T}_{\left[\gamma_{s k}\right]}}\right)$.

different fine scale parameters $\gamma_{i n}$. We compare the results with the respective single-level MFEM-WS $\left(\mathcal{E}_{\mathcal{R}} \mathcal{T}_{\left[\gamma_{s k}\right]}\right)$ methods, and show that the two-scale FE settings overcome the single-level one in all scenarios.

Assessing the effect of polynomial degree increment on errors

Figure 5 shows the cases for $k_{s k}=1$, with $h_{i n}=h_{s k} / 2$. The purpose is to analyze the effect of increasing $k_{i n}=k_{s k}+n, n=0,1$ and 2 .

As predicted in (5.6), the errors in $\underline{\underline{\sigma}}$ and $q$ are of order $k_{s k}+1$, independently of $n$, and these errors are about the same magnitude, in all the cases. $\overline{\bar{T}}$ he stress symmetry errors, which are proportional to the stress errors, also 

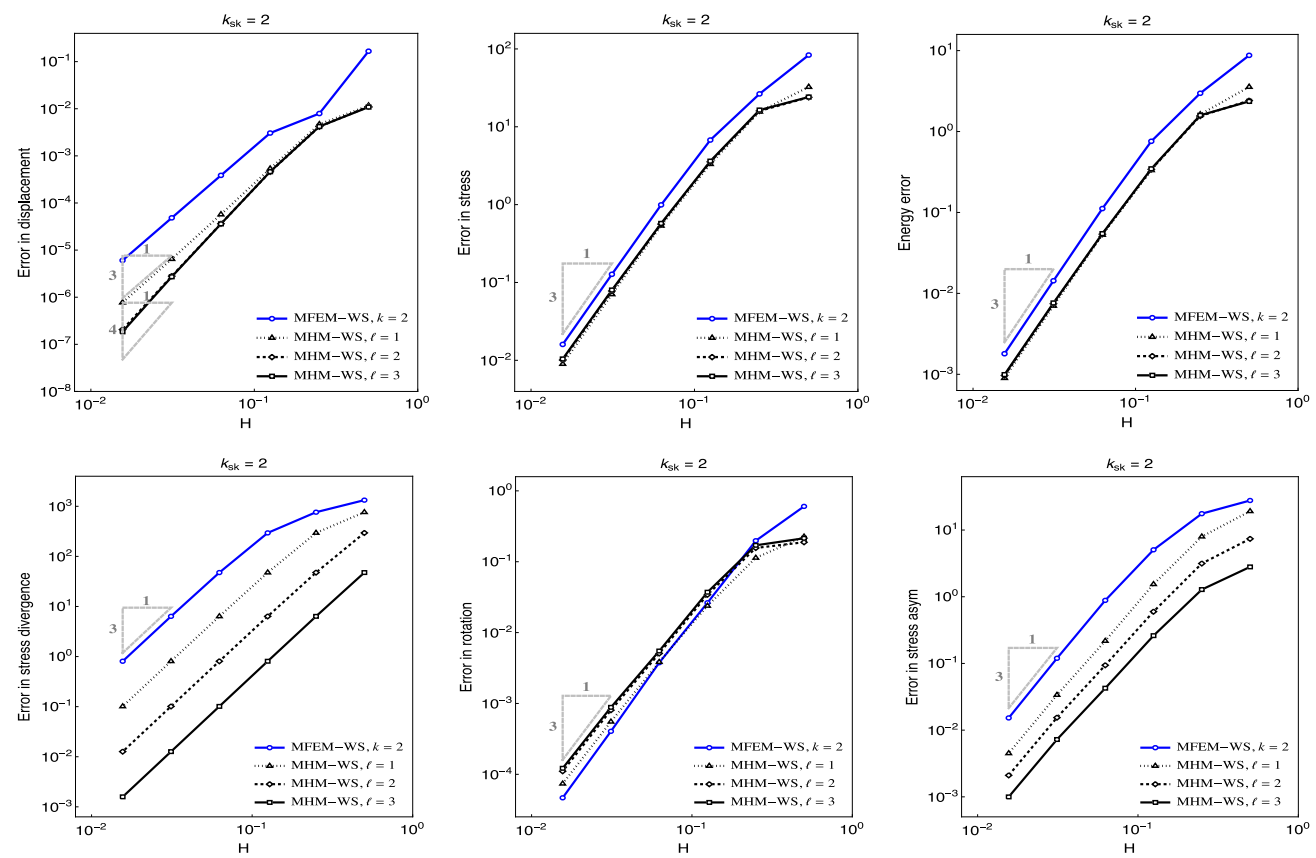

Figure 6. Problem 1 - Effect of decreasing $h_{i n}$ : MHM-WS $\left(\mathcal{E}_{\mathcal{R}} \mathcal{T}_{[\gamma]}\right)$ scheme with $k_{i n}=k_{s k}=2$, $h_{s k}=H=2^{-j}, j=1,2, \cdots 5, h_{i n}=h_{s k} / 2^{\ell}, \ell=1,2$, and 3; single-level MFEM-WS $\left(\mathcal{E}_{\left.\mathcal{R} \mathcal{T}_{\left[\gamma_{s k}\right]}\right)}\right.$.

have convergence rates of order $k_{s k}+1$, but the increment of the polynomial degrees inside the subregions reduces their magnitudes significantly. As expected, the divergence of the stress systematically improves accuracy to

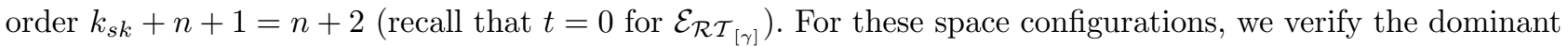
effect of the first term in the displacement error estimate (5.8) of order $k_{s k}+2$ when $n=1,2$. For $n=0$, i.e., when $k_{i n}=k_{s k}$, the second term of (5.8) takes place, and convergence rate of order $k_{s k}+1$ occurs, in accordance with the illustration of Figure 3 (left-plots).

\section{Assessing the effect of internal mesh refinement on errors}

Now we take $k_{i n}=k_{s k}=2$, and analyze the effect of refining the internal meshes, for $h_{\text {in }}=h_{s k} / 2^{\ell}, \ell=1,2$ and 3, comparing the cases in Figure 6. Again, for coarser levels $(\ell=1,2)$ the second term in the displacement error is the most significant, of order $k_{s k}+1$, in accordance with the left-plots of Figure 3. By further refining the internal grids $(\ell=3)$, the convergence rate tends to the order $k_{s k}+2$ related to the first term in (5.8). We highlight the different regimes for the divergence of the stress, which is now always of fixed order $k_{s k}+1=3$, but with reducing magnitude as $\ell$ increases. The behavior of the other variables are not significantly affected by using these FE space settings.

Comparison between $M H M-W S\left(\mathcal{E}_{\gamma}\right)$ and $M H M-H^{1}$ methods

We compare the convergence histories of the MHM-WS( $\left.\mathcal{E}_{\mathcal{R}} \mathcal{T}_{[\gamma]}\right)$ and MHM- $H^{1}$ methods in the plots of Figure 7 . The FE spaces have polynomial degree $k_{s k}=1$, without skeleton subdivision $\left(h_{s k}=H\right)$ and no internal polynomial degree enrichment $(n=0)$, but using $\ell=1,2$, and 3 to form the micro meshes inside the macro-elements $\left(h_{\text {in }}=H / 2^{\ell}\right)$.

For the MHM- $H^{1}$ method, using scalar polynomials obtained from $\mathbb{Q}_{k, k}(\hat{K}), L^{2}$-stress and energy errors maintain of order $k_{s k}$, but as the internal mesh refinement increases, the magnitude of the errors decrease. Concerning the displacement variable, both methods show similar behavior, starting with rates of order $k_{s k}+1$ 

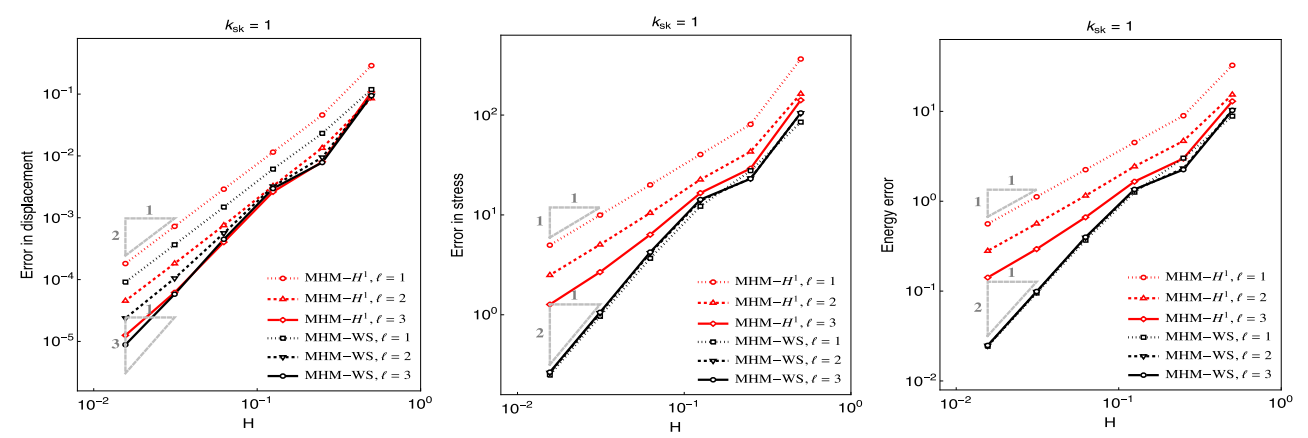

Figure 7. Problem 1 - Comparison of MHM-WS $\left(\mathcal{E}_{\mathcal{R} \mathcal{T}_{[\gamma]}}\right)$ and MHM- $H^{1}$ methods: $k_{i n}=k_{s k}=$ $1, h_{s k}=H=2^{-j}, j=1,2, \cdots, h_{\text {in }}=h_{s k} / 2^{\ell}, \ell=1,2$, and 3 .
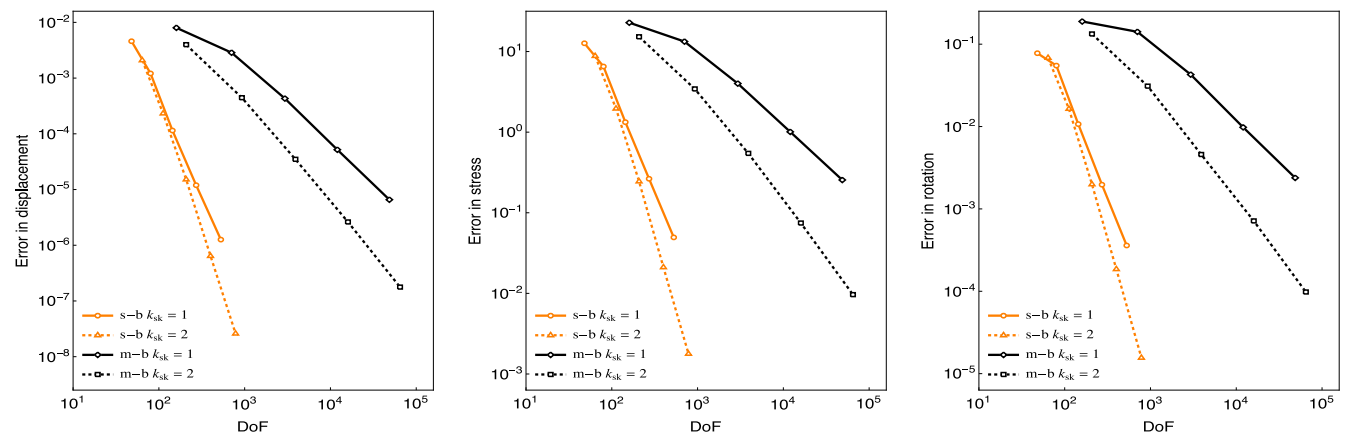

FiguRE 8. Problem 1 - Space-based convergence (s-b) for the MHM-WS $\left(\mathcal{E}_{\mathcal{R}} \mathcal{T}_{[\gamma]}\right)$ scheme: fixed macro-partition with 16 uniform square subregions, $h_{s k}=2^{-j}, j=2, \cdots, 6$, for $k_{s k}=1$ or 2 , $h_{i n}=h_{s k} / 2$, and $k_{i n}=k_{s k}+1$; mesh-based convergence $(\mathrm{m}-\mathrm{b})$ is for macro-partitions with $H=h_{s k}$.

at low internal refinement levels, typical of single-level schemes, as predicted by the error estimate in (5.8) for this kind of space configuration $\left(n=0, t=0\right.$, and $\left.h_{i n} \sim h\right)$, whose first term on the right side of $(5.8)$ is dominating.

However, as $\ell$ increases, with $h_{i n} \ll h_{s k}$, the error magnitudes decrease. The enhanced rate of order $k_{s k}+2$ is observed at $\ell=3$, illustrating the domination of the first term on the right side of (5.8).

Notice that the observed rates of convergence for the MHM- $H^{1}$ method are in accordance with the predicted ones in $[28,35]$, the errors from the local level solver polluting the global convergence when $k_{i n}=k_{s k}$. One can recover the higher convergence order $O\left(h_{s k}^{k_{s k}+1}\right)$ for $L^{2}$-stress and energy norms by using $k_{i n}=k_{s k}+1$ and, provided some smoothing properties hold, recover the super-convergence order $O\left(h_{s k}^{k_{s k}+2}\right)$ by using $k_{i n}=k_{s k}+2$ in the MHM- $H^{1}$ method [35].

\subsubsection{A space-based convergence study}

Consider now fixed macro-partitions with 16 uniform squares or 32 triangles, respectively (mesh-size $H=\frac{1}{4}$ ), and skeleton partitions taking $h_{s k}=2^{-j} H, j=0,1,2, \cdots$. Inside the subregions, we take uniform partitions $\mathcal{T}_{h_{i n}}^{\Omega_{i}}$ with $h_{i n}=h_{s k} / 2$. The polynomial degrees used for the trace spaces are $k_{s k}=1$ or 2 , and for the local FE spaces are $k_{i n}=k_{s k}+1$ (i.e., $n=1$ ). 
TABLE 3. Problem 1 - Space-based convergence for the MHM-WS $\left(\mathcal{E}_{\gamma}\right)$ method: $L^{2}$-errors and convergence rates using fixed macro-partition of 32 triangles for two-scale FE spaces $\mathcal{E}_{\mathcal{B D}} \mathcal{M}_{\gamma}$ and $\mathcal{E}_{\mathcal{B D M}_{\gamma}^{+}} ; h_{s k}=2^{-j}, k_{s k}=1$ or $2, h_{i n}=h_{s k} / 2$, and $k_{i n}=k_{s k}+1$.

\begin{tabular}{|c|c|c|c|c|c|c|c|c|c|c|c|c|}
\hline \multicolumn{13}{|c|}{$\begin{array}{l}\text { Triangular local partitions } \\
\mathcal{E}_{\mathcal{B D}^{\prime} \mathcal{M}_{\gamma}}\end{array}$} \\
\hline & \multicolumn{12}{|c|}{$k_{s k}=1$} \\
\hline \multirow[b]{2}{*}{$j$} & \multicolumn{2}{|c|}{ Stress } & \multicolumn{2}{|c|}{ Displacement } & \multicolumn{2}{|c|}{ Divergence } & \multicolumn{2}{|c|}{ Rotation } & \multicolumn{2}{|c|}{ Asymmetry } & \multicolumn{2}{|c|}{ Energy } \\
\hline & Error & Rate & Error & Rate & Error & Rate & Error & Rate & Error & Rate & Error & Rate \\
\hline 2 & $1.5298 \mathrm{e}+1$ & - & $6.1570 \mathrm{e}-3$ & - & $2.3282 \mathrm{e}+2$ & - & $1.5827 \mathrm{e}+0$ & - & $5.0094 \mathrm{e}+0$ & - & $1.5277 \mathrm{e}+0$ & - \\
\hline 3 & $3.8547 \mathrm{e}+0$ & 1.99 & $1.2550 \mathrm{e}-3$ & 2.29 & $6.6334 \mathrm{e}+1$ & 1.81 & $3.8729 \mathrm{e}-2$ & 2.03 & $1.3566 \mathrm{e}+0$ & 1.88 & $3.7603 \mathrm{e}-1$ & 2.02 \\
\hline 4 & $7.9571 \mathrm{e}-1$ & 2.28 & $2.8845 \mathrm{e}-4$ & 2.12 & $1.7167 \mathrm{e}+1$ & 1.95 & $7.2450 \mathrm{e}-3$ & 2.42 & $2.6268 \mathrm{e}-1$ & 2.37 & $7.6726 \mathrm{e}-2$ & 2.29 \\
\hline 5 & $1.6220 \mathrm{e}-1$ & 2.30 & $7.1211 \mathrm{e}-5$ & 2.02 & $4.3292 \mathrm{e}+0$ & 1.99 & $1.4674 \mathrm{e}-3$ & 2.30 & $4.8063 \mathrm{e}-2$ & 2.45 & $1.5543 \mathrm{e}-2$ & 2.30 \\
\hline & \multicolumn{12}{|c|}{$k_{s k}=2$} \\
\hline \multirow[b]{2}{*}{$j$} & \multicolumn{2}{|c|}{ Stress } & \multicolumn{2}{|c|}{ Displacement } & \multicolumn{2}{|c|}{ Divergence } & \multicolumn{2}{|c|}{ Rotation } & \multicolumn{2}{|c|}{ Asymmetry } & \multicolumn{2}{|c|}{ Energy } \\
\hline & Error & Rate & Error & Rate & Error & Rate & Error & Rate & Error & Rate & Error & Rate \\
\hline 2 & $6.5982 \mathrm{e}+0$ & - & $1.4763 \mathrm{e}-3$ & - & $1.0815 \mathrm{e}+1$ & - & $6.8016 \mathrm{e}-2$ & - & $1.4044 \mathrm{e}+0$ & -- & $6.4350 \mathrm{e}-1$ & - \\
\hline 3 & $1.1056 \mathrm{e}-1$ & 2.58 & $1.2802 \mathrm{e}-4$ & 3.53 & $1.0815 \mathrm{e}+1$ & 2.79 & $1.0762 \mathrm{e}-2$ & 2.66 & $1.9111 \mathrm{e}-1$ & 2.88 & $1.0699 \mathrm{e}-1$ & 2.59 \\
\hline 4 & $1.1581 \mathrm{e}-1$ & 3.25 & $8.1727 \mathrm{e}-6$ & 3.97 & $1.4003 \mathrm{e}+0$ & 2.95 & $1.1113 \mathrm{e}-3$ & 3.28 & $2.3519 \mathrm{e}-2$ & 3.02 & $1.1212 \mathrm{e}-2$ & 3.20 \\
\hline 5 & $9.4750 \mathrm{e}-3$ & 3.61 & $6.4039 \mathrm{e}-7$ & 3.67 & $1.7659 \mathrm{e}-1$ & 2.99 & $8.5089 \mathrm{e}-5$ & 3.71 & $2.6922 \mathrm{e}-3$ & 3.13 & $9.2499 \mathrm{e}-4$ & 3.60 \\
\hline \multicolumn{13}{|c|}{$\mathcal{E}_{\mathcal{B D} \mathcal{M}_{\gamma}^{+}}$} \\
\hline & \multicolumn{12}{|c|}{$k_{s k}=1$} \\
\hline \multirow[b]{2}{*}{$j$} & \multicolumn{2}{|c|}{ Stress } & \multicolumn{2}{|c|}{ Displacement } & \multicolumn{2}{|c|}{ Divergence } & \multicolumn{2}{|c|}{ Rotation } & \multicolumn{2}{|c|}{ Asymmetry } & \multicolumn{2}{|c|}{ Energy } \\
\hline & Error & Rate & Error & Rate & Error & Rate & Error & Rate & Error & Rate & Error & Rate \\
\hline 2 & $1.5909 \mathrm{e}+1$ & - & $4.7291 \mathrm{e}-3$ & - & $7.4825 \mathrm{e}+1$ & - & $1.9752 \mathrm{e}-1$ & - & $2.6632 \mathrm{e}+0$ & - & $1.5436 \mathrm{e}+0$ & - \\
\hline 3 & $4.0471 \mathrm{e}+0$ & 1.97 & $6.1283 e-4$ & 2.95 & $1.0815 \mathrm{e}+1$ & 2.79 & $4.8076 \mathrm{e}-2$ & 2.04 & $7.1458 \mathrm{e}-1$ & 1.90 & $3.8563 \mathrm{e}-1$ & 2.00 \\
\hline 4 & $8.2132 \mathrm{e}-1$ & 2.30 & $5.8335 \mathrm{e}-5$ & 3.39 & $1.4003 \mathrm{e}+0$ & 2.95 & $8.4784 \mathrm{e}-3$ & 2.50 & $1.2752 \mathrm{e}-1$ & 2.49 & $7.7762 \mathrm{e}-2$ & 2.31 \\
\hline 5 & $1.6348 \mathrm{e}-1$ & 2.33 & $6.3446 \mathrm{e}-6$ & 3.20 & $1.7659 \mathrm{e}+1$ & 2.99 & $1.5663 \mathrm{e}-3$ & 2.44 & $1.9175 \mathrm{e}-2$ & 2.73 & $1.5442 \mathrm{e}-2$ & 2.33 \\
\hline & \multicolumn{12}{|c|}{$k_{s k}=2$} \\
\hline \multirow{2}{*}{$j$} & Stres: & & Displacer & ent & Diverge & & Rotat & & Asymm & & Eners & \\
\hline & Error & Rate & Error & Rate & Error & Rate & Error & Rate & Error & Rate & Error & Rate \\
\hline 2 & $6.6848 \mathrm{e}+0$ & - & $1.4911 \mathrm{e}-3$ & - & $2.0206 \mathrm{e}+1$ & - & $7.3370 \mathrm{e}-2$ & - & $9.1499 \mathrm{e}+1$ & - & $6.4407 \mathrm{e}+1$ & - \\
\hline 3 & $1.1190 \mathrm{e}+0$ & 2.58 & $1.2639 \mathrm{e}-4$ & 3.56 & $1.4320 \mathrm{e}+0$ & 3.82 & $1.1316 \mathrm{e}-2$ & 2.70 & $1.1343 \mathrm{e}-1$ & 3.01 & $1.0775 \mathrm{e}-1$ & 2.58 \\
\hline 4 & $1.1724 \mathrm{e}-1$ & 3.25 & $6.8611 \mathrm{e}-6$ & 4.20 & $9.2555 \mathrm{e}-2$ & 3.95 & $1.1689 \mathrm{e}-3$ & 3.28 & $1.5131 \mathrm{e}-2$ & 2.91 & $1.1291 \mathrm{e}-2$ & 3.25 \\
\hline 5 & $9.6566 \mathrm{e}-3$ & 3.60 & $2.5853 \mathrm{e}-7$ & 4.73 & $5.8340 \mathrm{e}-3$ & 3.99 & $9.3046 \mathrm{e}-5$ & 3.65 & $1.7545 \mathrm{e}-3$ & 3.11 & $9.3336 \mathrm{e}-4$ & 3.60 \\
\hline
\end{tabular}

The space-based error curves in Figure 8 are for $\mathcal{E}_{\mathcal{R}} \mathcal{T}_{[\gamma]}$ spaces and are plotted versus the number of degrees of freedom (DoF) in the condensed systems of the upscaling stage. We also include mesh-based results for comparison, using the same grid size $H=h_{s k}$ for the macro and the skeleton partitions, while keeping the other parameters unchanged. These plots show that the desired accuracy can be obtained with about two orders of magnitude fewer DoFs when the space-based strategy is adopted instead of refining the global partition.

Table 3 contains the errors and convergence rates for triangular mesh scenarios. Recall the accuracy parameters $t=r=-1$ for the $\mathcal{E}_{\mathcal{B D} \mathcal{M}_{\gamma}}$ family, and $t=r=0$ for $\mathcal{E}_{\mathcal{B D} \mathcal{M}_{\gamma}^{+}}$case. Except for the divergence of the stress, all other variables experiment and enhanced accuracy, which is more evident for $k_{s k}=2$. Since $\underline{\nabla} \cdot \underline{\underline{\sigma}}$ is the $L^{2}$-orthogonal projection of $\underline{f}$ over $\mathscr{U}_{\gamma_{i n}}$, its accuracy is kept in the superconvergence rate of order $h_{i n}^{k_{s k}}{ }_{\overline{\overline{+}} t+2}$, as predicted in (5.7). As for the MHM- $H^{1}$ simulations shown in $[27,35]$, the $\mathcal{E}_{\mathcal{B D} \mathcal{M}_{\gamma}^{+}}$space family presents the same tendency to extrapolate the predicted values by an exponent $\approx 1 / 2$ in the space-based convergence rates. Once 

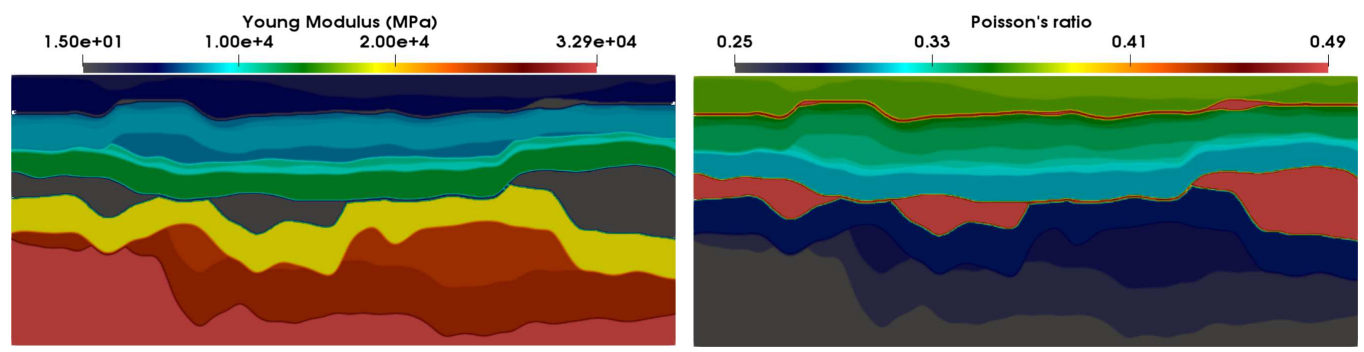

Figure 9. Problem 2 - Young's modulus and Poisson's ratio at the cross line $y=5000 \mathrm{~m}$.

stress convergence rate reaches the order $h_{s k}^{k_{s k}+3 / 2}$, this enhanced accuracy is translated to the displacement error estimate (5.8), improving the Term 1 to order $h_{s k}^{k_{s k}+5 / 2}$.

When this term is dominant, the extra $h_{s k}^{1 / 2}$ accuracy order appears, as observed for the $\mathcal{E}_{\mathcal{B D} \mathcal{M}_{\gamma}^{+}}$family using the mesh sizes and polynomial degree scenario of this test problem. However, this tendency is not confirmed by the displacement errors given by the simulations with $\mathcal{E}_{\mathcal{B D} \mathcal{M}_{\gamma}}$, a fact that can be justified by a closer look in the right-plots of Figure 3: using $n=1$ and $\ell=1$ the Term 2 (of order $h_{s k}^{k_{s k}+1}$ ) dominates the Term 1 as $h_{s k}$ diminishes.

\subsection{Problem 2: a heterogeneous media case}

In this example, we use the data from the HPC4e Test Suite [15], which defines an elastic domain with 16 layers with constant physical properties, covering an area of $10 \times 10 \times 5 \mathrm{~km}$. As suggested in [35], we replace the original layers 4 and 12 by the data of saturated clay $\rho=1760 \mathrm{~kg} / \mathrm{m}^{3}, \nu=0.49, E=15 \mathrm{MPa}$, and which adds more interesting behavior for the numerical experiments.

We consider a three-dimensional grid with $\Delta x=\Delta y=19.53125 \mathrm{~m}(10000 / 512)$ and $\Delta z=4500 / 256=$ $17.578125 \mathrm{~m}$, to sample the compressional velocity $V_{p}$, shear velocity $V_{s}$, and density $\rho$, and use the expressions $\nu=\frac{V_{p}^{2}-2 V_{s}^{2}}{2\left(V_{p}^{2}-V_{s}^{2}\right)}$ and $E=2 \rho V_{s}^{2}(1+\nu)$ to obtain the Poisson coefficient and Young's modulus, respectively. Figure 9 shows the plots of these parameters defined at the central cross line at $y=5000 \mathrm{~m}$, which corresponds to the domain $\Omega$ used in the simulations of the heterogeneous media case.

The top, left and right sides of the domain are stress-free, and the bottom side has zero displacement. The domain is loaded by gravity $\left(9.81 \mathrm{~m} / \mathrm{s}^{2}\right)$. We choose the evolution of $\underline{\underline{\sigma}}_{11}$ at the horizontal centerline of the domain $z=2250.25 \mathrm{~m}$ as a reference value.

We ran single-level MFEM-WS $\left(\mathcal{E}_{\mathcal{R} \mathcal{T}_{\left[\gamma_{r e f}\right]}}\right)$ simulations for $\gamma_{r e f}=(h, k), k=1,2$ and conclude that $k=2$ can be used as a reference solution.

The plots in Figure 10 show the reference values of $\underline{\sigma}_{11}$ along the horizontal center line of the domain $z=2250.25 \mathrm{~m}$ for four two-level configurations of the $\overline{\mathrm{M}} \mathrm{HM}-\mathrm{WS}\left(\mathcal{E}_{\mathcal{R}} \mathcal{T}_{[\gamma]}\right)$ using fixed $16 \times 8$ subregions as documented in the right-hand side of Figure 11. The interior meshes in each subregion are obtained after five uniform subdivisions. The approximations are obtained using FE spaces $\mathcal{E}_{\mathcal{R} \mathcal{T}_{[\gamma]}}$ for $k_{s k}=k_{i n}=1$, and $h_{s k}=H / 2^{\ell}, \ell=0,1,2$ and 3 divisions of the skeleton mesh. It can be observed that by refining the skeleton mesh, the MHM-WS $\left(\mathcal{E}_{\mathcal{R} \mathcal{T}_{[\gamma]}}\right)$ approximations become closer to the reference solution, with quite well matching for $\ell=3$.

Figure 11 shows contour plots of $\underline{\underline{\sigma}}_{11}$ comparing the re ference results (left-image) with the finest MHM$\mathrm{WS}\left(\mathcal{E}_{\mathcal{R} \mathcal{T}_{[\gamma]}}\right)$ approximate result (right-image) using $\ell=3$. 

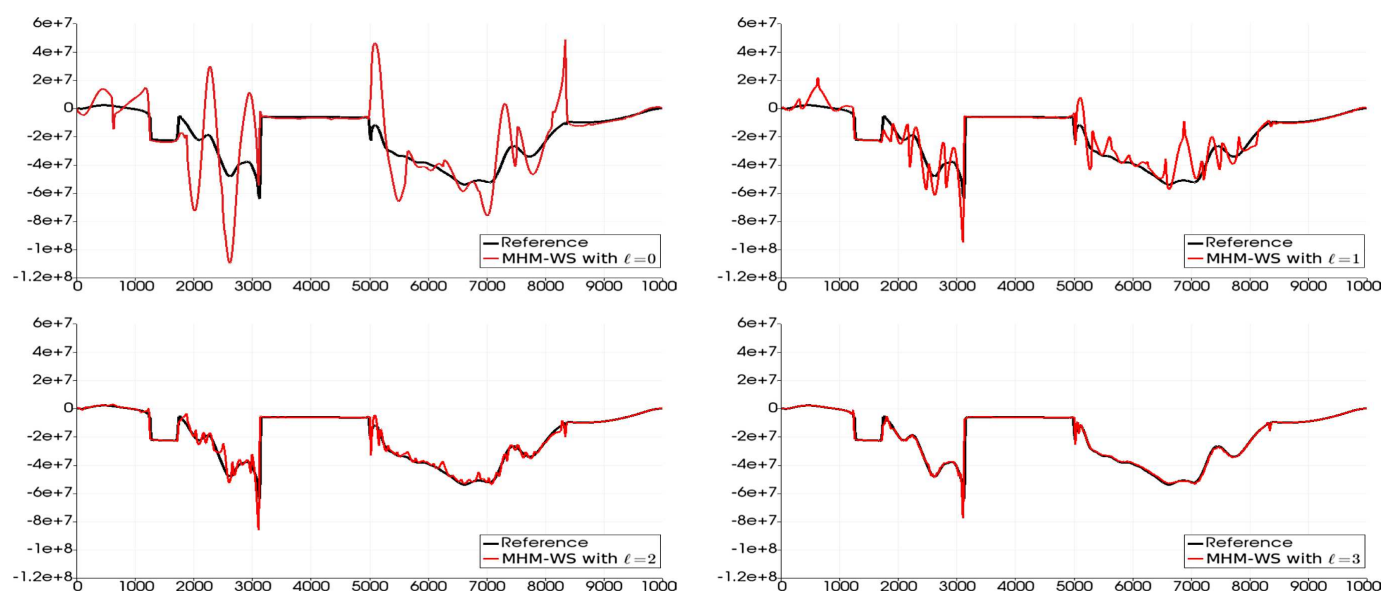

FIgURE 10. Problem 2 - Plots of $\underline{\underline{\sigma}}_{11}$ at the height $z=2250.25 \mathrm{~m}$. The reference approximations (black), and the $\operatorname{MHM}-\mathrm{WS}\left(\mathcal{E}_{\gamma}\right)$ solutions (red), are for two-scale FE spaces $\mathcal{E}_{\mathcal{R}} \mathcal{T}_{[\gamma]}$ based on $16 \times 8$ macro subregions, $k_{s k}=k_{i n}=1, h_{\text {in }}=10000 / 512 \approx 19.53 \mathrm{~m}, h_{s k}=H / 2^{\ell}$ with $\ell=0,1,2,3$.
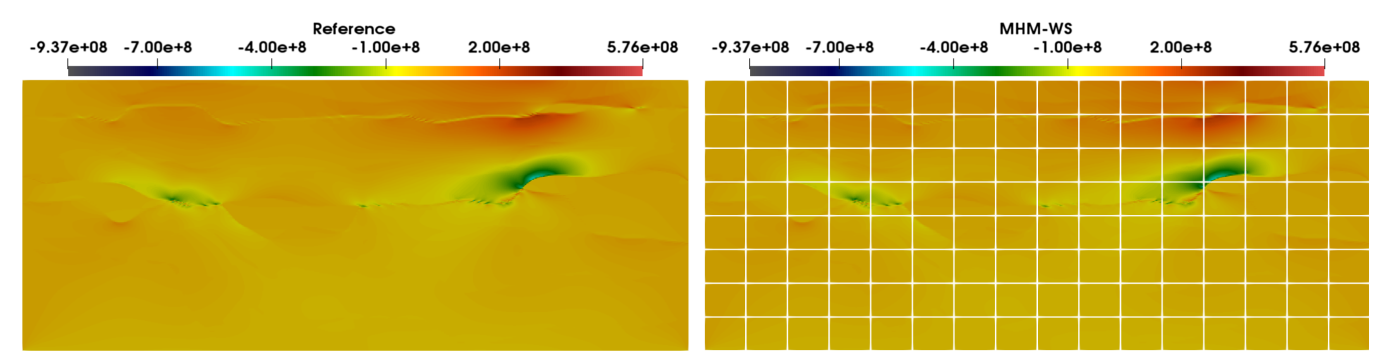

FiguRE 11. Problem 2 - 3 Component $\underline{\underline{\sigma}}_{11}$ obtained with single-level reference FE space

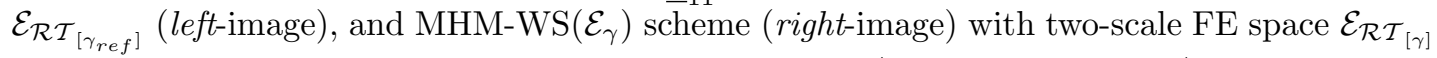
based on $16 \times 8$ macro-elements, $k_{s k}=k_{i n}=1, h_{s k}=H / 8$, and $h_{i n}=10000 / 512 \approx 19.53 \mathrm{~m}$.

\section{Conclusions}

We proposed a family of stable $H$ (div)-conforming multiscale mixed methods for elasticity problems that impose weakly stress symmetry on general polygonal meshes. Such a feature makes the methodology flexible to represent complex geometries while it yields a systematic way to build multiscale FE spaces with upscalingdownscaling stages. The multiscale nature of the methods provides a detailed representation of the solution (stress, displacement, and rotation multiplier). Such discrete solutions combine fine-scale computations within macro elements, which are entirely independent of one another and prompt to be parallelized, with coarse scales represented by constrained traction (Lagrange multiplier) on mesh skeleton. As a result, the methods achieve optimal and high-order convergence by refining the meshes' frame and local sub-meshes only. As an upshot, the convergence also holds with edge refinement only, i.e., keeping the first-level mesh fixed. Interestingly, we observed numerically super-convergence in this case. Also, local stress fields are in local equilibrium with external forces. Those properties are theoretically demonstrated and validated through numerical tests, which verified the robustness of the methods on a highly complex multilayer problem using meshes non-aligned with interface layers. 
We conclude with the highlight on the following topics deserving future research:

- The MHM methodology can provide an underlying algorithm with two levels of parallelism. The first one has a mathematical origin, based on the local-global splitting in (3.6)-(3.13) and (3.14), (3.15), respectively. The second one is computational, based on the choice of parallel algorithms to solve each problem (global and local ones), and the management of computational resources to deliver efficient code. A study on this direction demands expertise on the implementation of parallel algorithms, which is out of the scope of the present manuscript. We refer to $[25,35]$ as some seminal works considering the performance of the MHM- $\mathrm{H}^{1}$ method for elasticity.

- The construction of a two-scale MHM-WS characterization for three-dimension MFEM-WS methods is feasible using a similar methodology, as in the 2D case presented in Theorem 3.2. Recall that, stable singlelevel FE spaces for the MFEM-WS formulation are available in 3D only for tetrahedral geometry (e.g., in $[3,6,13,21,40])$. However, stability analyses for them should use a different methodology, for the application of the Stokes-compatibility constraint is less effective in 3D.

\section{Appendix A. Proof of theorems}

\section{A.1. Proof of Theorem 2.1}

Theorem 2.1 gives the characterization of the weak stress mixed formulation with reduced symmetry (2.2)(2.4) in terms of the local-global hybrid systems (2.7)-(2.10), (2.11)-(2.14), and (2.15), (2.16). Before going throughout its proof, let the mapping $R_{r m}: \Lambda \rightarrow \mathscr{U}_{r m}$ be defined by $\left(R_{r m}(\underline{\lambda}), \underline{v}\right)_{\Omega_{i}}=\left\langle\delta_{i} \underline{\lambda}, \underline{v}\right\rangle_{\partial \Omega_{i}}, \underline{\forall} \in \mathscr{U}_{r m}$. If $\left(\underline{u}_{r m}, \underline{\lambda}\right)$ solves the global system $(2.15)$ and $(2.16)$, then for (2.7) and (2.16) we obtain $-\underline{\nabla} \cdot T^{\underline{\sigma}}(\underline{\lambda})=R_{r m}(\lambda)=$ $\Pi^{r m}(\underline{f}), \Pi^{r m}(\underline{f})$ denoting the $L^{2}$-orthogonal projection of $\underline{f} \in \mathscr{U}$ onto $\mathscr{U}_{r m}$.

Lemma A.1. The mapping $R_{r m}$ is a surjective operator.

Proof. In fact, given $\underline{v}^{*} \in \mathscr{U}_{r m}$, let $\left.\underline{\lambda}^{*}\right|_{\partial \Omega_{i}}=\left.\underline{\underline{\sigma}}^{*} \underline{n}\right|_{\partial \Omega_{i}} \in \Lambda$, where $\underline{\underline{\sigma}}^{*} \in H^{1}(\Omega, \mathbb{S})$ satisfy $\underline{\nabla} \cdot \underline{\underline{\sigma}}^{*}=\underline{v}^{*}$. Thereby, $\left\|\underline{v}^{*}\right\|_{L^{2}\left(\Omega, \mathbb{R}^{2}\right)}^{2}=\sum_{\Omega_{i}}\left(\underline{v}^{*}, \underline{v}^{*}\right)_{\Omega_{i}}=\sum_{\Omega_{i}}\left\langle\underline{\underline{\sigma}}^{*} \underline{n}^{\Omega^{\Omega}}, \underline{v}^{*}\right\rangle_{\partial \Omega_{i}}=\sum_{\Omega_{i}}\left\langle\delta_{i} \underline{\lambda}^{*}, \underline{v}^{*}\right\rangle_{\partial \Omega_{i}}=\sum_{\Omega_{i}}\left\langle R_{r m}\left(\underline{\bar{\lambda}}^{*}\right), \underline{v}^{*}\right\rangle_{\partial \Omega_{i}}$. Then, the adjoint application of $R_{r m}$ is injective with closed range, which implies the result.

The results of Theorem 2.1 shall be proved by parts.

Part 1. Notice that the variables $T^{\underline{\sigma}}(\underline{\lambda}) \in H(\operatorname{div}, \Omega, \mathbb{M}), T^{\underline{u}}(\underline{\lambda}) \in \mathscr{U}^{\perp}$, and $T^{q}(\underline{\lambda}) \in L^{2}\left(\Omega_{i}\right)$, provided by the local mixed solvers (2.7)-(2.10), can be interpreted as solution, in the distributional sense, of the independent local boundary value problems for $\left.\underline{u}\right|_{\Omega_{i}}$ free of rigid body modes:

$$
-\underline{\nabla} \cdot \underline{\underline{\sigma}}=R_{r m}(\underline{\lambda}), \quad \underline{\underline{A}}^{-1} \underline{\underline{\sigma}}=\nabla \underline{u}-\underline{\underline{\gamma}}(\underline{u}), \quad \underline{\underline{\sigma}}-\underline{\underline{\sigma}}^{T}=0 \text { in } \Omega_{i},\left.\quad \underline{\underline{\sigma}} \underline{\underline{n}}\right|_{\partial \Omega_{i}}=\underline{\lambda},
$$

with balanced force $R_{r m}(\underline{\lambda})$ and Neumann boundary condition $\underline{\lambda}$. Thus, their corresponding solutions are unique. In fact, Neumann boundary value problems of linear elasticity are singular, with kernel formed by the rigid motions of the body. However, this ambiguity can be removed by enforcing the solution to be free of rigid body modes, and by requiring balanced force and Neumann boundary terms (e.g., see [31]).

Analogously, $\hat{T}^{\underline{\sigma}}(f) \in \mathscr{S}, \hat{T}^{\underline{u}}(f) \in \mathscr{U}^{\perp}$, and $\hat{T}^{q}(f) \in \mathscr{Q}$, are obtained from the unique weak solutions of the local problems

$$
-\underline{\nabla} \cdot \underline{\underline{\sigma}}=\underline{f}-\Pi^{r m}(\underline{f}), \quad \underline{\underline{A}}^{-1} \underline{\underline{\sigma}}=\nabla \underline{u}-\underline{\underline{\gamma}}(\underline{u}), \quad \underline{\underline{\sigma}}-\underline{\underline{\sigma}}^{T}=0 \text { in } \Omega_{i},\left.\quad \underline{\underline{\sigma}} \underline{\underline{n}}\right|_{\partial \Omega_{i}}=0 .
$$

To verify the uniqueness of the global system (2.15) and (2.16), take zero data $\underline{f}=0$ and $\underline{g}=0$. Then it becomes

$$
\begin{array}{rlrl}
\left(\underline{\underline{A}}^{-1} T^{\underline{\sigma}}(\underline{\lambda}), T^{\underline{\sigma}}(\underline{\mu})\right)+\left(\underline{u}_{r m}, \underline{\nabla} \cdot T^{\underline{\sigma}}(\underline{\mu})\right) & =0, & & \forall \underline{\mu} \in \Lambda, \\
\left(\underline{\nabla} \cdot T^{\underline{\sigma}}(\underline{\lambda}), \underline{v}\right) & =0, & \forall \underline{v} \in \mathscr{U}_{r m} .
\end{array}
$$


Taking test functions $\underline{\mu}=\underline{\lambda}$ and $\underline{v}=\underline{\nabla} \cdot T \underline{\underline{\sigma}}(\underline{\lambda}) \in \mathscr{U}_{r m}$, these equations turn into

$$
\begin{array}{r}
\left(\underline{\underline{A}}^{-1} T^{\underline{\sigma}}(\underline{\lambda}), T^{\underline{\sigma}}(\underline{\lambda})\right)+\left(\underline{u}_{r m}, \underline{\nabla} \cdot T^{\underline{\sigma}}(\underline{\lambda})\right)=0, \\
\left(\underline{\nabla} \cdot T^{\underline{\sigma}}(\underline{\lambda}), \underline{\nabla} \cdot T^{\underline{\sigma}}(\underline{\lambda})\right)=0,
\end{array}
$$

implying that $\underline{\nabla} \cdot T^{\underline{\sigma}}(\underline{\lambda})=0$, from which $\left.\left(\underline{\underline{A}}^{-1} T^{\underline{\sigma}}(\underline{\lambda}), T \stackrel{\underline{\sigma}}{(\underline{\lambda}}\right)\right)=0$ holds. The positive definiteness of the tensor $\underline{\underline{A}}$ implies that $T \underline{\underline{\sigma}}(\underline{\lambda})=0$ (meaning that $\underline{\lambda}=0, T^{\underline{u}}(\underline{\lambda})=0$, and $T^{q}(\underline{\lambda})=0$ as well). Finally, $\underline{u}_{r m}=0$ follows from the remaining relation $\left(\underline{u}_{r m}, \underline{\nabla} \cdot T_{\underline{\underline{\sigma}}}^{\underline{\sigma}}(\underline{\mu})\right)=0, \forall \underline{\mu} \in \Lambda$, recalling that $\underline{\nabla} \cdot T^{\underline{\sigma}}(\underline{\mu})=-R_{r m}(\underline{\mu})$, and that $R_{r m}(\mu)$ is a surjective operator over $\mathscr{U}_{r m}$.

Part 2. Suppose $\left(\underline{u}_{r m}, \underline{\lambda}\right) \in \mathscr{U}_{r m} \times \Lambda$ solves $(2.15),(2.16)$, and let $(\underline{\underline{\sigma}}, \underline{u}, q)$ be recovered as in (2.5). If $(\underline{\underline{s}}, \underline{w}, r)$ is the solution of $(2.2)-(2.4)$, define $\underline{\nu}=\left.\underline{s} \underline{n}\right|_{\Gamma}$ and set $\underline{w}=\underline{w}_{r m}+\underline{w}^{\perp}$.

By testing (2.3) with $\underline{v} \in \mathscr{U}^{\perp}$, and $(2.2)$ with $\underline{\underline{\tau}} \in \dot{\mathscr{S}}$, both with support in $\Omega_{i}$, and recalling that $\left(w_{r m}, \underline{\nabla}\right.$. $\underline{\underline{\tau}})_{\Omega_{i}}=-\frac{1}{2}\left(\operatorname{asym} \nabla \underline{w}_{r m}, \operatorname{asym} \underline{\underline{\tau}}\right)_{\Omega_{i}}$, we obtain

$$
\begin{aligned}
\left(\underline{\underline{A}}^{-1} \underline{\underline{s}}, \underline{\underline{\tau}}\right)_{\Omega_{i}}+\left(\underline{w}^{\perp}, \underline{\nabla} \cdot \underline{\underline{\tau}}\right)_{\Omega_{i}}+\left(r-\frac{1}{2} \operatorname{asym} \nabla \underline{w}_{r m}, \operatorname{asym} \underline{\underline{\tau}}\right)_{\Omega_{i}} & =0 \\
-(\underline{\nabla} \cdot \underline{\underline{s}}, \underline{v})_{\Omega_{i}} & =(\underline{f}, \underline{v})_{\Omega_{i}} .
\end{aligned}
$$

For arbitrary $\mu \in \Lambda$, take $\underline{\underline{\tau}}=T^{\underline{\sigma}}(\mu)$ to test $(2.2)$. Notice that $(r$ asym $T \stackrel{\underline{\sigma}}{=}(\underline{\mu}))=0$ (for $\left.(2.9)\right)$, and $\left(\underline{w}^{\perp}, \underline{\nabla}\right.$. $T \stackrel{\underline{\sigma}}{\underline{\rho}}(\underline{\mu}))=0$ (for $(2.7))$. Then equations $(2.2)$ and $(2.3)$ become

$$
\begin{aligned}
\left(\underline{\underline{A}}^{-1} \underline{\underline{s}}, T \underline{\underline{\sigma}}(\underline{\mu})\right)+\left(\underline{w}_{r m}, \underline{\nabla} \cdot T \underline{\underline{\sigma}}(\underline{\mu})\right) & =\langle\underline{\mu}, \underline{g}\rangle, \\
-(\underline{\nabla} \cdot \underline{\underline{s}}, \underline{v}) & =(\underline{f}, \underline{v}), \quad \forall \underline{v} \in \mathscr{U}_{r m} .
\end{aligned}
$$

By confronting (A.1)-(A.4) and (2.2) with equations (2.7)-(2.14), the differences $\underline{\underline{s}}-\underline{\underline{\sigma}}, \underline{w}^{\perp}-\underline{u}^{\perp}$, and $r-q$ verify:

$$
\begin{aligned}
& (\underline{\nabla} \cdot[\underline{\underline{s}}-\underline{\underline{\sigma}}], \underline{v})_{\Omega_{i}}=0, \quad \forall \underline{v} \in \mathscr{U}^{\perp}\left(\Omega_{i}\right), \\
& \left(\underline{\underline{A}}^{-1}[\underline{\underline{s}}-\underline{\underline{\sigma}}], \underline{\underline{\tau}}\right)_{\Omega_{i}}+\left(\underline{w}^{\perp}-\underline{u}^{\perp}, \underline{\nabla} \cdot \underline{\underline{\tau}}\right)_{\Omega_{i}}+(r-q, \operatorname{asym} \underline{\underline{\tau}})_{\Omega_{i}}=0, \quad \forall \underline{\underline{\tau}} \in \dot{\mathscr{S}}\left(\Omega_{i}\right) \\
& (\operatorname{asym}[\underline{\underline{s}}-\underline{\sigma}], \varphi)_{\Omega_{i}}=0, \quad \forall \varphi \in L^{2}\left(\Omega_{i}\right), \\
& \left.(\underline{\underline{s}}-\underline{\underline{\sigma}}) \underline{n}\right|_{\partial \Omega_{i}}=\left.(\underline{\nu}-\underline{\lambda})\right|_{\partial \Omega_{i}} \text {. }
\end{aligned}
$$

These equations imply that $\underline{\underline{s}}-\underline{\underline{\sigma}}=T \underline{\underline{\sigma}}(\underline{\nu}-\underline{\lambda}), \underline{w}^{\perp}-\underline{u}^{\perp}=T^{\underline{u}}(\underline{\nu}-\underline{\lambda})$, and $r-q=T^{q}(\underline{\nu}-\underline{\lambda})$.

Furthermore, since equations (2.11), (2.8), and (2.13) imply that

$$
\begin{aligned}
\left(\underline{f}, T^{\underline{u}}(\underline{\mu})\right)_{\Omega_{i}} & =-\left(\underline{\nabla} \cdot \hat{T}^{\underline{\sigma}}(\underline{f}), T^{\underline{u}}(\underline{\mu})\right)_{\Omega_{i}} \\
& =\left(\underline{\underline{A}}^{-1} T^{\underline{\sigma}}(\underline{\mu}), \hat{T}^{\underline{\sigma}}(\underline{f})\right)_{\Omega_{i}}+\left(T^{q}(\underline{\mu}), \operatorname{asym} \hat{T}^{\underline{\sigma}}(\underline{f})\right)_{\Omega_{i}} \\
& =\left(\underline{\underline{A}}^{-1} T^{\underline{\sigma}}(\underline{\mu}), \hat{T}^{\underline{\sigma}}(\underline{f})\right)_{\Omega_{i}}=\left(T^{\underline{\sigma}}(\underline{\mu}), \underline{\underline{A}}^{-1} \hat{T}^{\underline{\sigma}}(\underline{f})\right)_{\Omega_{i}},
\end{aligned}
$$

and by recalling that $\left(\underline{\nabla} \cdot T^{\underline{\sigma}}(\underline{f}), \underline{v}\right)=0, \forall \underline{v} \in \mathscr{U}_{r m}$, we conclude from (2.15), (2.16) and (A.3), (A.4) that

$$
\begin{aligned}
\left(\underline{\underline{A}}^{-1}[\underline{\underline{s}}-\underline{\underline{\sigma}}], T \underline{\underline{\sigma}}(\underline{\mu})\right)+\left(\underline{w}_{r m}-\underline{u}_{r m}, \underline{\nabla} \cdot T \underline{\underline{\sigma}}(\underline{\mu})\right) & =0, \forall \underline{\mu} \in \Lambda, \\
-(\underline{\nabla} \cdot[\underline{\underline{s}}-\underline{\sigma}], \underline{v}) & =0, \quad \forall \underline{v} \in \mathscr{U}_{r m} .
\end{aligned}
$$


By setting $\underline{\mu}=\underline{\nu}-\underline{\lambda}$ and $\underline{v}=\underline{w}_{r m}-\underline{u}_{r m}$ in (A.9) and (A.10), and using the positive definiteness property of $\underline{\underline{A}}^{-1}$, we conclude that $\underline{\underline{s}}=\underline{\underline{\sigma}}$ (i.e., $(\underline{\nu}=\underline{\lambda})$. Thus $\underline{w}^{\perp}=\underline{u}^{\perp}$ and $r=q$ as well. Finally, equation (A.9) becomes $\left(\underline{w}_{r m}-\underline{u}_{r m}, \underline{\nabla} \cdot \stackrel{\bar{T}}{\underline{\underline{\sigma}}(\underline{\mu}))}=0, \forall \underline{\bar{\mu}} \in \Lambda\right.$, implying that $\underline{w}_{r m}=\underline{u}_{r m}$, from Remark (2). By uniqueness of the solutions in both contexts, the equivalence property holds, and then the existence of a solution for (2.15) and (2.16) follows from the existence of a solution for (2.2)-(2.4).

\section{A.2. Proof of Theorem 3.1}

The goal is to prove uniqueness of solution for the $\operatorname{MHM}-\mathrm{WS}\left(\mathcal{E}_{\gamma}\right)$ scheme. The next result is paramount for its proof.

Lemma A.2. The mapping $\tilde{R}_{r m}: \Lambda_{\gamma} \rightarrow \mathscr{U}_{r m}$, defined by $\tilde{R}_{r m}=\left.R_{r m}\right|_{\Lambda_{\gamma}}$, is surjective.

Proof. Recall that, for $\mu \in \Lambda_{\gamma},\left(\tilde{R}_{r m}(\mu), \underline{v}\right)_{\Omega_{i}}=\left\langle\delta_{i} \mu, \underline{v}\right\rangle_{\partial \Omega_{i}}, \forall \underline{v} \in \mathscr{U}_{r m}$. As in the proof of Lemma A.1, given $\underline{v}^{*} \in \mathscr{U}_{r m}$, let $\underline{\underline{\sigma}}^{*} \in H^{1}(\bar{\Omega}, \mathbb{S})$ satisfying $\underline{\nabla} \cdot \underline{\underline{\sigma}}^{*}=\underline{v}^{*}$. Then, define $\underline{\tilde{\lambda}}^{*}=\left.\underline{\underline{\sigma}}^{*} \underline{n}\right|_{\partial \Omega_{i}}, \Omega_{i} \in \mathcal{T}$, where $\underline{\underline{\tilde{\sigma}}}^{*}=\Pi_{1, \gamma}^{\underline{\underline{\sigma}}} \underline{\underline{\sigma}}^{*} \in$ $\mathscr{S}_{\gamma}$, and the interpolant $\Pi_{1, \gamma}^{\underline{\underline{\sigma}}}$, defined for $\underline{\underline{\tau}} \in H^{1}(\Omega, \mathbb{M})$, is such that $\left(\underline{\nabla} \cdot\left(\underline{\underline{\tau}}-\Pi_{1, \gamma}^{\underline{\underline{\sigma}}}\right), \underline{v}\right)=0, \forall \underline{v} \in \mathscr{U}_{\gamma_{i n}}$. The existence of such mapping is stated in Theorem 5.1. Thus, $\underline{\tilde{\lambda}}^{*} \in \Lambda_{\gamma}$, and the assumption $\mathscr{U}_{r m} \subset \mathscr{U}_{\gamma_{i n}}$ implies that $\underline{\nabla} \cdot \underline{\underline{\sigma}}^{*}=\underline{v}^{*}$. Consequently, $\left(\underline{\tilde{v}}^{*}, \underline{v}\right)_{\Omega_{i}}=\left\langle\underline{\underline{\sigma}}^{*} \underline{n}^{\Omega_{i}}, \underline{v}\right\rangle_{\partial \Omega_{i}}=\left\langle\delta_{i} \underline{\tilde{\lambda}}^{*}, \underline{v}\right\rangle_{\partial \Omega_{i}}$, meaning that $\tilde{R}_{r m}\left(\underline{\tilde{\lambda}}^{*}\right)=\underline{v}^{*}$, and the result follows.

By hypothesis, the downscaling solvers (3.6)-(3.9) are well-posed MFEM-WS $\left(\mathcal{E}_{\gamma}\right)$ versions in $\Omega_{i}$. Thus, uniqueness holds for $\left(\tilde{T} \underline{\underline{\sigma}}(\underline{\tilde{\lambda}}), \tilde{T}^{\underline{u}}(\underline{\lambda}), \tilde{T}^{q}(\underline{\lambda})\right) \in \mathscr{S}_{\gamma} \times \mathscr{U}_{\gamma_{i n}} \times \mathscr{Q}_{\gamma_{i n}}$.

Analogously, $\left(\tilde{\hat{T}} \underline{\underline{\sigma}}(\underline{f}), \tilde{\hat{T}}^{\underline{u}}(\underline{f}), \tilde{\hat{T}}^{q}(f)\right) \in \mathscr{S}_{\gamma} \times \mathscr{U}_{\gamma_{i n}} \times \mathscr{Q}_{\gamma_{i n}}$ is the unique solution piecewise defined by well-posed $\operatorname{MFEM}-\mathrm{WS}\left(\mathcal{E}_{\gamma}\right)$ formulations (3.10)-(3.13) in $\Omega_{i}$.

Uniqueness for the solution of the upscaling stage follows by similar proof steps as observed for the weak formulations at the continuous level. Taking zero data $\underline{f}=0$ and $\underline{g}=0$, the well-posedness of the local problems (3.10)-(3.13) implies that $\tilde{\hat{T}}^{\underline{u}}(\underline{f})=0, \tilde{\hat{T}}^{\underline{\sigma}}(\underline{f})=0$ (and $\overline{\hat{T}}^{q}(\underline{f})=0$ ). Then, the upscaling system becomes

$$
\begin{aligned}
\left(\underline{\underline{A}}^{-1} \tilde{T}^{\underline{\sigma}}(\underline{\tilde{\lambda}}), \tilde{T}^{\underline{\sigma}}(\underline{\mu})\right)+\left(\underline{\tilde{u}}_{r m}, \underline{\nabla} \cdot \tilde{T}^{\underline{\sigma}}(\underline{\mu})\right) & =0, \quad \forall \underline{\mu} \in \Lambda_{\gamma}, \\
(\underline{\nabla} \cdot \tilde{T} \underline{\underline{\sigma}}(\underline{\tilde{\lambda}}), \underline{v}) & =0, \quad \forall \underline{v} \in \mathscr{U}_{r m} .
\end{aligned}
$$

Testing with $\underline{\mu}=\underline{\tilde{\lambda}}$ and $\underline{v}=\underline{\nabla} \cdot \tilde{T} \underline{\underline{\sigma}}(\underline{\tilde{\lambda}}) \in \mathscr{U}_{r m}$ (for (3.6)), these equations turn into

$$
\begin{array}{r}
\left(\underline{\underline{A}}^{-1} \tilde{T}^{\underline{\sigma}}(\underline{\tilde{\lambda}}), \tilde{T}^{\underline{\sigma}}(\underline{\tilde{\lambda}})\right)+\left(\underline{\underline{u}}_{r m}, \underline{\nabla} \cdot \tilde{T}^{\underline{\sigma}}(\underline{\tilde{\lambda}})\right)=0, \\
\left(\underline{\nabla} \cdot \tilde{T}^{\underline{\sigma}}=(\underline{\lambda}), \underline{\nabla} \cdot \tilde{T}^{\underline{\sigma}}(\underline{\tilde{\lambda}})\right)=0,
\end{array}
$$

implying that $\underline{\nabla} \cdot \tilde{T} \stackrel{\underline{\sigma}}{=}(\underline{\tilde{\lambda}})=0$, from which $\left(\underline{\underline{A}}^{-1} \tilde{T}^{\underline{\sigma}}(\underline{\tilde{\lambda}}), \tilde{T}^{\underline{\sigma}}(\underline{\tilde{\lambda}})\right)=0$ holds. The positive definiteness of the tensor $\underline{\underline{\underline{A}}}$ implies that $\tilde{T}^{=}(\underline{\tilde{\lambda}})=0$, meaning that $\underline{\tilde{\lambda}}=0$ (and thus $\tilde{T}^{\underline{u}}(\underline{\tilde{\lambda}})=0$ and $\tilde{T}^{q}(\underline{\tilde{\lambda}})=0$ as well). Thus, the remaining relation is $\left(\underline{\tilde{u}}_{r m}, \underline{\nabla} \cdot \tilde{T} \underline{\underline{\sigma}}(\underline{\mu})\right)=0, \forall \underline{\mu} \in \tilde{\Lambda}_{\gamma}$. Noting that $-\underline{\nabla} \cdot \tilde{T} \underline{\underline{\sigma}}(\underline{\mu})=\tilde{R}_{r m}(\underline{\mu})$ in $\Omega_{i}$, for $\underline{\mu} \in \tilde{\Lambda}_{\gamma}$, and by Lemma A.2 there exists $\underline{\mu}^{*} \in \Lambda_{\gamma} \operatorname{such}$ that $\tilde{R}_{r m}\left(\underline{\mu}^{*}\right)=\underline{\tilde{u}}_{r m}$, we conclude that $\underline{\tilde{u}}_{r m}=0$, and the result follows.

\section{A.3. Proof of Theorem 3.2}

This is a discrete version of the equivalence result in Theorem 2.1 and the proof follows similar steps. Firstly, suppose $\left(\underline{\tilde{\lambda}}, \underline{\tilde{u}}_{r m}\right)$ solves the upscaling system of the $\operatorname{MHM}-\mathrm{WS}\left(\mathcal{E}_{\gamma}\right)$ method, and consider the recovered solution of the downscaling stage $\underline{\underline{\tilde{\sigma}}}=\tilde{T}^{\underline{\sigma}}(\underline{\tilde{\lambda}})+\tilde{\hat{T}}^{\underline{\sigma}}(\underline{f}), \underline{\tilde{u}}^{\perp}=\tilde{T}^{\underline{u}}(\underline{\tilde{\lambda}})+\tilde{\hat{T}}^{\underline{u}}(\underline{f})$, and $\tilde{q}=\frac{1}{2} \operatorname{asym} \underline{\tilde{u}}_{r m}+\tilde{T}^{q}(\underline{\tilde{\lambda}})+\tilde{\hat{T}}^{q}(\underline{f})$. After 
the combination of the systems (3.6)-(3.9) and (3.10)-(3.13), we obtain the following set of equations in the subregions:

$$
\begin{aligned}
-(\underline{\nabla} \cdot \underline{\underline{\tilde{\sigma}}}, \underline{v})_{\Omega_{i}} & =(\underline{f}, \underline{v})_{\Omega_{i}}, & & \forall \underline{v} \in \tilde{\mathscr{U}}_{\gamma_{i n}}^{\perp}\left(\Omega_{i}\right) . \\
\left(\underline{\underline{A}}^{-1} \underline{\underline{\tilde{\sigma}}}, \underline{\underline{\tau}}\right)_{\Omega_{i}}+\left(\underline{\tilde{u}^{\perp}}, \underline{\nabla} \cdot \underline{\tau}\right)_{\Omega_{i}}+\left(\tilde{q}-\frac{1}{2} \operatorname{asym} \nabla \tilde{u}_{r m}, \operatorname{asym} \underline{\underline{\tau}}\right)_{\Omega_{i}} & =0, & & \underline{\underline{\tau}} \in \dot{\mathscr{S}}_{\gamma}\left(\Omega_{i}\right), \\
(\operatorname{asym} \underline{\underline{\underline{\sigma}}}, \varphi)_{\Omega_{i}} & =0, & & \forall \varphi \in \mathscr{Q}_{\gamma_{i n}}\left(\Omega_{i}\right), \\
\left.\underline{\underline{\tilde{\sigma}}} \underline{\underline{n}}\right|_{\partial \Omega_{i}} & =\left.\underline{\tilde{\lambda}}\right|_{\partial \Omega_{i} .} & &
\end{aligned}
$$

On the other hand side, let $(\underline{\underline{\tilde{s}}}, \underline{\tilde{w}}, \tilde{r}) \in \mathcal{E}_{\gamma}$ be the MFEM-WS $\left(\mathcal{E}_{\gamma}\right)$ solution, and set $\underline{\tilde{\underline{v}}}=\left.\underline{\underline{\tilde{s}}} \underline{\underline{n}}\right|_{\Gamma}$, and $\underline{\tilde{w}}=$ $\underline{\tilde{w}}_{r m}+\underline{\tilde{w}}^{\perp}$. By confronting the above system of equations with similar one valid for $(\underline{\underline{s}}, \underline{\tilde{w}}, \tilde{r})$, we conclude that $\underline{\underline{\tilde{\sigma}}}-\underline{\underline{\tilde{s}}}=\tilde{T} \underline{\tilde{\sigma}}(\underline{\tilde{\lambda}}-\underline{\tilde{v}}), \underline{\tilde{u}}^{\perp}-\underline{\tilde{w}}^{\perp}=\tilde{T}^{\underline{u}}(\underline{\tilde{\lambda}}-\underline{\tilde{v}})$, and $\tilde{q}-\tilde{r}=\tilde{T}^{q}(\underline{\tilde{\lambda}}-\underline{\tilde{v}})$. For arbitrary $\mu \in \Lambda_{\gamma},(3.10),(3.7)$, and (3.12) imply that $(\underline{f}, \tilde{T} \underline{u}(\underline{\mu}))_{\Omega_{i}}=\left(\tilde{T}^{\underline{\sigma}}(\underline{\mu}), \underline{\underline{A}}^{-1} \tilde{\hat{T}}^{\underline{\sigma}}(\underline{f})\right)_{\Omega_{i}}$. Using this relation and the properties $\left.\underline{\mu}\right|_{\partial \Omega}=\tilde{T}^{\underline{\sigma}}(\underline{\mu}) \underline{n}^{\Omega}$ (by (3.13)), and $(\underline{\nabla} \cdot \tilde{\hat{T}} \underline{\underline{\sigma}}(\underline{f}), \underline{v})=0, \forall \underline{v} \in \mathscr{U}_{r m}$, equations (3.14) and (3.15) become

$$
\begin{aligned}
\left(\underline{\underline{A}}^{-1} \underline{\underline{\tilde{\sigma}}}, \tilde{T}^{\underline{\sigma}}(\underline{\mu})\right)+\left(\underline{\tilde{u}}_{r m}, \underline{\nabla} \cdot \underline{\tilde{T}} \underline{\underline{\sigma}}(\underline{\mu})\right) & =\langle\underline{\mu}, \underline{g}\rangle, & & \forall \underline{\mu} \in \Lambda_{\gamma}, \\
-(\underline{\nabla} \cdot \underline{\underline{\sigma}}, \underline{v}) & =(\underline{f}, \underline{v}), & & \forall \underline{v} \in \mathscr{U}_{r m} .
\end{aligned}
$$

Inserting in equation $(3.16)$ the facts $(\tilde{r}$, asym $T \underline{\underline{\sigma}}(\underline{\mu}))=0$, and $\left(\underline{\tilde{w}}^{\perp}, \underline{\nabla} \cdot T \stackrel{\underline{\sigma}}{\underline{\mu}}(\underline{)})=0\right.$, and recalling equation (3.17), we obtain

$$
\begin{array}{rlrl}
\left(\underline{\underline{A}}^{-1} \underline{\underline{\tilde{s}}}, T \stackrel{\sigma}{=}(\underline{\mu})\right)+\left(\underline{\tilde{w}}_{r m}, \underline{\nabla} \cdot T \stackrel{\sigma}{=}(\underline{\mu})\right)=\langle\underline{\mu}, \underline{g}\rangle, & & \forall \underline{\mu} \in \Lambda_{\gamma} \\
-(\underline{\nabla} \cdot \underline{\underline{s}}, \underline{v}) & =(\underline{f}, \underline{v}), & & \forall \underline{v} \in \mathscr{U}_{r m}
\end{array}
$$

Consequently,

$$
\begin{aligned}
\left(\underline{\underline{A}}^{-1}[\underline{\underline{\tilde{\sigma}}}-\underline{\tilde{s}}], \tilde{T}^{\underline{\sigma}}(\underline{\mu})\right)+\left(\underline{\tilde{u}}_{r m}-\tilde{w}_{r m}, \underline{\nabla} \cdot \tilde{T} \stackrel{\tilde{\sigma}}{=}(\underline{\mu})\right) & =0, & & \forall \underline{\mu} \in \Lambda_{\gamma} . \\
-(\underline{\nabla} \cdot[\underline{\underline{\tilde{\sigma}}}-\underline{\tilde{s}}], \underline{v}) & =0, & & \forall \underline{v} \in \mathscr{U}_{r m} .
\end{aligned}
$$

By setting $\underline{\mu}=\underline{\tilde{\lambda}}-\underline{\tilde{v}}$ and $\underline{v}=\underline{\tilde{u}}_{r m}-\underline{\tilde{w}}_{r m}$ in the above relations, and since we already know that $\underline{\underline{\tilde{\sigma}}}-\underline{\underline{\tilde{s}}}=$ $\tilde{T} \stackrel{\sigma}{=}(\underline{\tilde{\lambda}}-\underline{\tilde{\nu}})$, the positive definiteness property of $\underline{\underline{A}}^{-1}$ implies that $\underline{\underline{\sigma}}=\underline{\underline{s}}($ i.e., $\underline{\tilde{\lambda}}=\underline{\tilde{\nu}})$. Thus $\underline{\tilde{u}}^{\perp}=\underline{w}^{\perp}$ and $\tilde{q}=\tilde{r}$ as well. Finally, the remaining equation $\left(\underline{\tilde{u}}_{r m}-\underline{\tilde{\tilde{w}}}_{r m}, \underline{\nabla} \cdot T^{\underline{\sigma}}(\mu)\right)=\underline{\overline{0}}, \underline{\bar{\forall}} \underline{\underline{\mu}} \in \Lambda_{\gamma}$, and Lemma A.2, concerning the surjectivity over $\mathscr{U}_{r m}$ of $\underline{\nabla} \cdot \tilde{T} \stackrel{\underline{\sigma}}{=}(\underline{\mu})=-R_{r m}(\underline{\mu}), \mu \in \Lambda_{\gamma}$, imply that $\underline{\tilde{u}}_{r m}=\underline{\tilde{w}}_{r m}$. By uniqueness of MHM-WS$\left(\mathcal{E}_{\gamma}\right)$ and $\operatorname{MFEM}-\mathrm{WS}\left(\mathcal{E}_{\gamma}\right)$ solutions, these methods are equivalent.

\section{Appendix B. List of Symbols}

\begin{tabular}{llll}
\hline \multicolumn{3}{c}{ Acronyms for FE methods } \\
\hline NAME & Poisson FE pairs & NAME & Stokes FE pairs \\
\hline $\mathcal{B D} \mathcal{M}$ & Brezzi-Douglas-Marini for triangles & $\mathcal{C} \mathcal{R}$ & Crouzeix-Raviart for triangles \\
$\mathcal{R} \mathcal{T}$ & Raviart-Thomas for quadrilaterals & $\mathcal{G} \mathcal{R}$ & Girault-Raviart for quadrilaterals \\
\hline \multicolumn{4}{c}{ Elasticity methods } \\
\hline MFEM-WS & Mixed FE method weakly imposing tensor symmetry \\
MHM-WS & Multiscale hybrid mixed FE method weakly imposing tensor symmetry \\
MHM-H $^{1}$ & Primal multiscale hybrid mixed FE method \\
$\mathcal{E}_{N A M E_{\gamma}}$ & Tensor and displacement rows come from Poisson FE pair NAME \\
\hline
\end{tabular}




\begin{tabular}{|c|c|c|c|c|c|}
\hline \multicolumn{6}{|c|}{ Scalars, vectors and tensors } \\
\hline$\underline{A}$ & Stiffness tensor & $\sigma$ & Stress tensor & $\tilde{\sigma}$ & Approximate $\sigma$ \\
\hline & Identity matrix & $\overline{\bar{u}}$ & Displacement & $\underline{\overline{\tilde{u}}}$ & Approximate $\underline{\bar{u}}$ \\
\hline$\overline{\overline{\mathrm{M}}}$ & Second-order tensors & $\overline{\bar{u}}_{r m}$ & Rigid body mode & $\overline{\tilde{u}}_{r m}$ & Approximate $\underline{\bar{u}}_{r m}$ \\
\hline & Symmetric tensors & $u^{\perp}$ & $L^{2}$-complement of $\underline{u}_{r m}$ & $\underline{\tilde{u}}^{\perp}{ }^{\perp}$ & Approximate $\underline{u}^{\perp}$ \\
\hline$\underline{\underline{\varepsilon}}$ & Strain tensor & $\underline{\bar{\lambda}}$ & Multiplier (traction) & $\underline{\tilde{\lambda}}$ & Approximate $\underline{\lambda}$ \\
\hline & & $\bar{q}$ & Rotation & $\overline{\tilde{q}}$ & Approximate $\bar{q}$ \\
\hline \multicolumn{6}{|c|}{ Data } \\
\hline$\underline{f}$ & Body force & $\mu$ & Lamé's second parameter & $E$ & Young's modulus \\
\hline $\bar{g}$ & Boundary data & $\lambda$ & Lamé's first parameter & $\nu$ & Poisson's ratio \\
\hline
\end{tabular}

\begin{tabular}{llll}
\hline & & Geometry & \\
\hline$\Omega \subset \mathbb{R}^{2}$ & Polygonal domain & $\mathcal{T}=\left\{\Omega_{i}\right\}$ & Macro-partition of $\Omega$ \\
$\partial \Omega$ & Boundary of $\Omega$ & $\mathcal{T}_{h_{s k}}$ & Coarse conformal partition of $\Omega$ \\
$\Omega_{i}$ & Subregions of $\Omega$ & $\mathcal{T}_{h_{i}}^{\Omega_{i}}$ & Coarse partition of $\Omega_{i}$ \\
$\partial \Omega_{i}$ & Boundary of $\Omega_{i}$ & $\mathcal{T}_{h_{i}}$ & Refined partition of $\Omega_{i}$ \\
$\Gamma=\left\{\partial \Omega_{i}\right\}$ & Mesh skeleton & $\mathcal{T}^{\Gamma}$ & Coarse partition of $\Gamma$ \\
$D \subset \Omega$ & Subdomain & $\hat{K}$ & Master element \\
$\underline{n}$ & Normal vector field & $K$ & Element in $\mathcal{T}_{h_{i}}^{\Omega_{i}}$ \\
$\underline{n}^{D}$ & Outward unit normal & $\bar{K}$ & Element in $\mathcal{T}_{h_{s k}}^{\Omega_{i}}$ \\
\hline
\end{tabular}

\begin{tabular}{llll}
\hline \multicolumn{3}{c}{ Functional spaces } \\
\hline$L^{2}(D)$ & Scalar $L^{2}$-space & $\mathscr{S}=H(\operatorname{div}, \Omega, \mathbb{M})$ & Tensor H(div)-space for $\underline{\underline{\sigma}}$ \\
$L^{2}(D, \mathbb{E})$ & $L^{2}$-space $\mathbb{E} \in\left\{\mathbb{R}^{2}, \mathbb{M}\right\}$ & $\mathscr{\mathscr { S }} \subset \mathscr{S}$ & Bubble tensors \\
$(,)_{D}$ & $L^{2}$ inner product & $\mathscr{U}=L^{2}\left(\Omega, \mathbb{R}^{2}\right)$ & Space for displacement $\underline{u}$ \\
$H^{s}(D)$ & Scalar Sobolev space & $\mathscr{U}_{r m} \subset \mathscr{U}$ & Rigid body modes \\
$H^{s}(D, \mathbb{E})$ & Sobolev space $\mathbb{E} \in\left\{\mathbb{R}^{2}, \mathbb{M}\right\}$ & $\mathscr{U}^{\perp} \subset \mathscr{U}$ & $L^{2}$-orthogonal complement of $\mathscr{U}_{r m}$ \\
$H(\operatorname{div}, D)$ & Vector $H(\operatorname{div})$-space & $\mathscr{Q}=L^{2}(\Omega)$ & Space for rotation $q$ \\
$H(\operatorname{div}, D, \mathbb{M})$ & Tensor $H(\operatorname{div})$-space & $\Lambda=\Lambda\left(\Gamma, \mathbb{R}^{2}\right)$ & Normal trace space for $\underline{\lambda}$ \\
$H^{1 / 2}\left(\partial D, \mathbb{R}^{2}\right)$ & Trace of $H(\operatorname{div}, D, \mathbb{M})$ & $\langle\rangle$, & Duality pairing of traces \\
$H^{-1 / 2}\left(\partial D, \mathbb{R}^{2}\right)$ & Trace of $H^{1}\left(\Omega, \mathbb{R}^{2}\right)$ & & \\
\hline
\end{tabular}

\begin{tabular}{lll}
\hline \multicolumn{3}{c}{ Downscaling operators } \\
\hline$T(\underline{\lambda}): \Lambda \rightarrow \mathscr{S} \times \mathscr{U}^{\perp} \times \mathscr{Q}$ & $\tilde{T}(\tilde{\tilde{\lambda}})$ & Discrete version of $T(\underline{\lambda})$ \\
$T(\underline{f}): \Lambda \rightarrow \mathscr{S} \times \mathscr{U}^{\perp} \times \mathscr{Q}$ & $\tilde{\hat{T}}(\underline{f})$ & Discrete version of $\hat{T}(\underline{f})$ \\
\hline
\end{tabular}


Finite element spaces

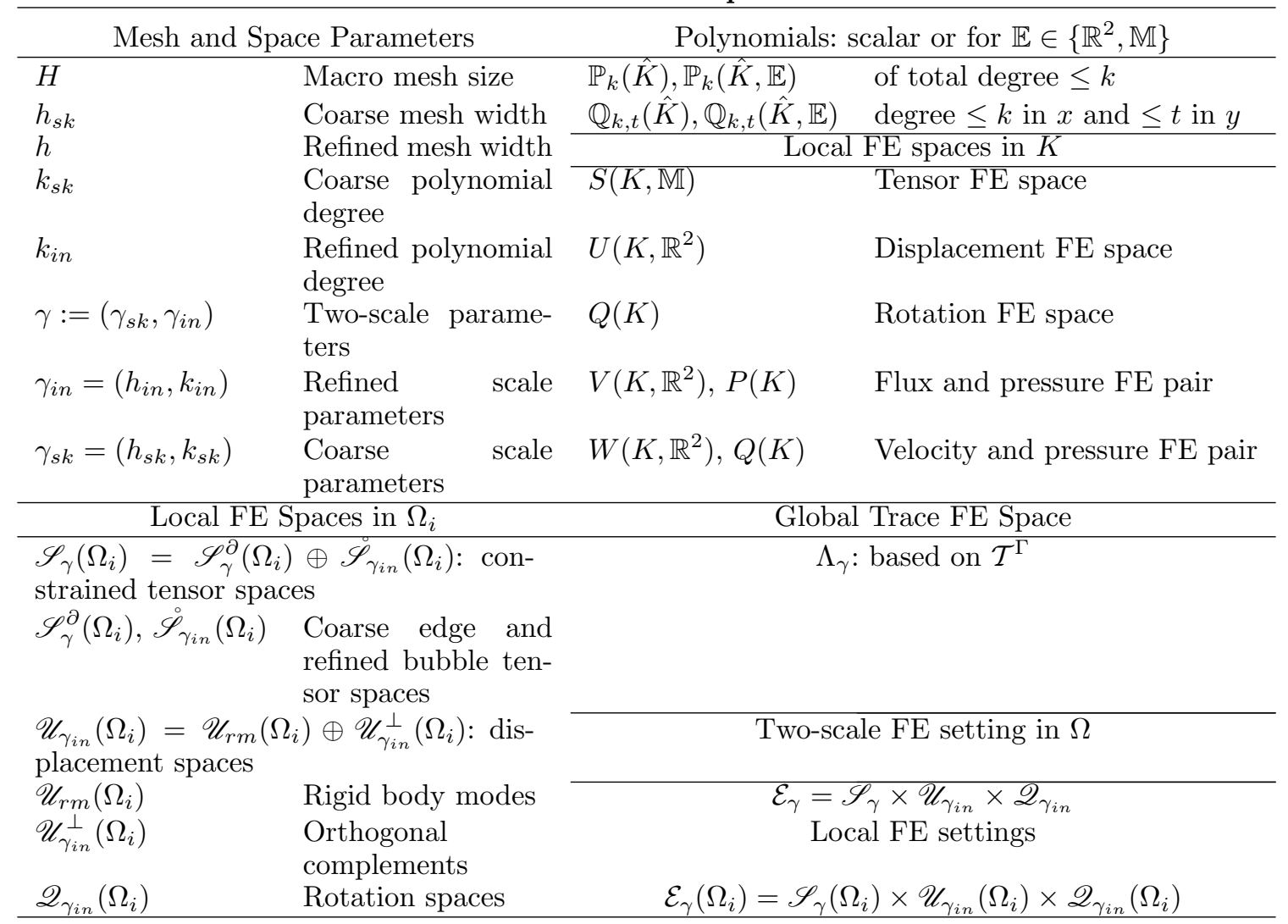

Acknowledgements. The authors P. R. B. Devloo and S. M. Gomes thankfully acknowledges financial support from CNPq - Conselho Nacional de Desenvolvimento Científico e Tecnológico/Brazil (grants 305823-2017-5, and 306167/2017-4, respectively). P. R. B. Devloo also acknowledges financial support from ANP - Brazilian National Agency of Petroleum, Natural Gas and Biofuels (grant 2014/00090-2). S. M. Gomes is grateful for the support and hospitality during her visit to Universidade Federal da Paraíba, PB, Brazil, whilst this manuscript was partialy prepared. F. Valentin has received funding from the European Union's Horizon 2020 Programme (2014-2020), from the MCTI - Brazilian Ministry of Science, Technology and Innovation, through RNP - Rede Nacional de Pesquisa, under the HPC4E Project (www. hpc4e.eu, grant 689772), from CAPES - Coordenaçāo de Aperfeiçoamento de Pessoal de Nivel Superior/Brazil, under the PHOTOM and EOLIS projects within the Math/Amsud program, and CNPq/Brazil (grant 309173/2020-5). W. S. Pereira was funded by the MCTI/RNP/Brazil under the HPC4E Project, the CNPq/Brazil (grant 140764/2015-1), and the company Bull Ltda and the SCAC - Cooperation and Cultural Action Service, from the French Embassy in Brazil under the CIFRE program.

\section{REFERENCES}

[1] A. Abdulle, Analysis of a heterogeneous multiscale FEM for problems in elasticity. Math. Models Methods Appl. Sci. 16 (2006) $615-635$.

[2] D.N. Arnold, D. Boffi and R.S. Falk, Quadrilateral H(div) finite elements. SIAM J. Numer. Anal. 42 (2005) $2429-2451$.

[3] D.N. Arnold, R.S. Falk and R. Winther, Mixed finite element methods for linear elasticity with weakly imposed symmetry. Math. Comp. 76 (2007) 1699-1723.

[4] D.N. Arnold, G. Awanou and R. Winther, Finite elements for symmetric tensors in three dimensions. Math. Comp. 77 (2008) $1229-1251$.

[5] D.N. Arnold, G. Awanou, B.W. Bestbury and W. Qiu, Mixed finite elements for elasticity on quadrilateral meshes. Adv. Comput. Math. 41 (2015) 553-572.

[6] D. Boffi, F. Brezzi and M. Fortin, Reduced symmetry elements in linear elasticity. Commun. Pure Appl. Anal. 8 (2009) 95-121. 
[7] D. Boffi, F. Brezzi and M. Fortin, Mixed Finite Element Methods and Applications. Springer Series in Computational Mathematics. Springer-Verlag, New York (2013).

[8] F. Brezzi, On the existence, uniqueness and approximation of saddle-point problems arising from lagrangian multipliers. RAIRO: Anal. Numér. 2 (1974) 129-151.

[9] F. Brezzi and J. Pitkäranta, On the stabilization of finite element approximations of the Stokes equations. In: Efficient Solutions of Elliptic Systems, edited by W. Hackbusch. Braunschweig, Wiesbaden (1984) 11-19.

[10] F. Brezzi, J. Douglas and L.D. Marini, Two families of mixed finite elements for second order elliptic problems. Numer. Math. 47 (1985) 217-235.

[11] M. Buck, O. Iliev and H. Andrä, Multiscale finite element coarse spaces for the application to linear elasticity. Cent. Eur. J. Math. 11 (2013) 608-701.

[12] M. Buck, O. Iliev and H. Andrä, Multiscale finite elements for linear elasticity: oscillatory boundary conditions. In Lect. Not. Comp. Sci. Springer International Publishing (2014) 237-245.

[13] B. Cockburn, J. Gopalakrishnan and J. Guzmán, A new elasticity element made for enforcing weak stress symmetry. Math. Comp. 79 (2010) 1331-1349.

[14] M. Crouzeix and P.A. Raviart, Conforming and nonconforming finite element methods for solving the stationary Stokes equations I. ESAIM:M2AN 7 (1973) 33-75.

[15] J. de la Puente, HPC4E Seismic Test Suite. https://hpc4e.eu/downloads/datasets-and-software (2016).

[16] L. Demkowicz, Polynomial exact sequences and projection-based interpolation with application to Maxwell equations. In: Mixed Finite Elements, Compatibility Conditions, and Applications, edited by D. Boffi and L. Gastaldi. Vol. 1939 of Lecture Notes in Mathematics. Springer, Berlin, Heidelberg (2008) 101-158.

[17] P.R.B. Devloo, C.M.A.A. Bravo and E.C. Rylo, Systematic and generic construction of shape functions for $p$-adaptive meshes of multidimensional finite elements. Comput. Meth. Appl. Mech. Eng. 198 (2009) 1716-1725.

[18] P.R.B. Devloo, A.M. Farias and S.M. Gomes, A remark concerning divergence accuracy order for $H$ (div)-conforming finite element flux approximations. Comput. Math. Appl. 77 (2019) 1864-1872.

[19] P.R.B. Devloo, S.M. Gomes, T. Quinelato and S. Tian, Enriched two dimensional mixed finite element models for linear elasticity with weak stress symmetry. Comput. Math. App. 79 (2020) 2678-2700.

[20] O. Durán, P.R.B. Devloo, S.M. Gomes and F. Valentin, A multiscale hybrid method for Darcy's problems using mixed finite element local solvers. Comput. Meth. Appl. Mech. Eng. 354 (2019) 213-244.

[21] Y. Efendiev and Y. Hou, Multiscale Finite Element Methods: Theory and Applications, Tutorials in the Applied Mathematical Sciences. Springer, New York 4 (2009).

[22] R.S. Falk, Finite element methods for linear elasticity. In: Mixed Finite Elements, Compatibility Conditions, and Applications, edited by D. Boffi and L. Gastaldi. Vol. 1939 of Lecture Notes in Mathematics. Springer, Berlin, Heidelberg (2008) 159-194.

[23] M. Farhloul and M. Fortin, Dual hybrid methods for the elasticity and the Stokes problems: a unified approach. Numer. Math. 76 (1997) 419-440.

[24] V. Girault and P.A. Raviart, Finite Element Methods for Navier-Stokes Equations: Theory and Algorithms. Springer Series in Computational Mathematics. Springer-Verlag, New York (1991).

[25] A.T.A. Gomes, D. Paredes, W.D.S. Pereira, R.P. Souto and F. Valentin, Performance analysis of the MHM simulator in a petascale machine. In: Proceedings of the XXXVIII Iberian Latin American Congress on Computational Methods in Engineering. ABMEC (2017).

[26] C. Harder, D. Paredes and F. Valentin, A family of multiscale hybrid-mixed finite element methods for the darcy equation with rough coefficients. J. Comput. Phys. 245 (2013) 107-130.

[27] C. Harder, D. Paredes and F. Valentin, On a multiscale hybrid-mixed method for advective-reactive dominated problems with heterogenous coefficients. SIAM Multiscale Model. Simul. 3 (2015) 491-518.

[28] C. Harder, A.L. Madureira and F. Valentin, A hybrid-mixed method for elasticity. ESAIM:M2AN 50 (2016) $311-336$.

[29] P. Henning and A. Persson, A multiscale method for linear elasticity reducing poisson locking. Comput. Meth. Appl. Mech. Eng. 310 (2016) 156-171.

[30] E. Khattatov and I. Yotov, Domain decomposition and multiscale mortar mixed finite elements methods for linear elasticity with weak stress symmetry. Math. Model. Numer. Anal. 53 (2019) 2081-2108.

[31] M. Kuchta, K.-A. Mardal and M. Mortensen, On the singular Neumann problem in linear elasticity. Numer. Linear. Algebra Appl. 26 (2019) e2212.

[32] A. Målqvist and D. Peterseim, Localization of elliptic multiscale problems. Math. Comp. 83 (2014) $2583-2603$.

[33] L. Mansfield, Finite element subspaces with optimal rates of convergence for the stationary Stokes problem. RAIRO Anal. Numer. 16 (1982) 49-66.

[34] D. Paredes, F. Valentin and H.M. Versieux, On the robustness of multiscale hybrid-mixed methods. Math. Comput. 86 (2016) $525-548$.

[35] W.S. Pereira, Multiscale hybrid-mixed methods for heterogeneous elastic models. Ph.D. thesis. LNCC, RJ, BR (2019).

[36] W. Pereira and F. Valentin, A locking-free MHM method for elasticity. In: Vol. 5 of Proceeding Series of the Brazilian Society of Computational and Applied Mathematics (2017).

[37] P.A. Raviart and J.M. Thomas, Primal hybrid finite element methods for 2nd order elliptic equations. Math. Comp. 31 (1997) 391-413.

[38] J.E. Roberts and J.-M. Thomas, Mixed and hybrid methods. In: Handbook of Numerical Analysis, edited by P.G. Ciarlet and J.L. Lions. Elsevier Science Publishers (1991) 527-639. 
[39] D. Siqueira, P.R.B. Devloo and S.M. Gomes, A new procedure for the construction of hierarchical high order $H$ div and $H$ curl finite element spaces. J. Comput. Appl. Math. 240 (2013) 204-214.

[40] R. Stenberg, A family of mixed finite elements for the elasticity problem. Numer. Math. 53 (1988) 513-538. 